\title{
Sec1p/Munc18 (SM) proteins and their role in regulating secretion in Saccharomyces cerevisiae and Caenorhabditis elegans a comparative approach
}

\author{
Dissertation \\ For the award of the degree \\ “Doctor rerum naturalium” (Dr. Rer. Nat.) \\ Division of Mathematics and Natural Sciences \\ Of the Georg-August-Universität Göttingen
}

Submitted by
Raul Emilio Iraheta

Born in

San Salvador, El Salvador

Göttingen, 2012 
Thesis Committee Members:

Prof. Dr. Dirk Fasshauer

Prof. Dr. Markus Wahl

Prof. Dr. Ralf Ficner
Department of Fundamental Neurosciences University of Lausanne Switzerland

Department of Chemistry and Biochemistry Freie University Berlin Germany

Department of Molecular Structural Biology Georg-August-Universitat Gottingen Germany 


\section{Declaration of Authorship}

Hereby, I confirm that I have created this work "Sec1p/Munc18 (SM) proteins and their role in regulating secretion in Saccharomyces cerevisiae and Caenorhabditis elegans a comparative approach"

entirely on my own and that I have only used the sources and material cited.

Göttingen, $3^{\text {rd }}$ of October 2012

Raul Emilio Iraheta 


\section{Acknowledgments}

I am indebt to Prof. Dr. Dirk Fasshauer for his support and guidance that I have received during my graduate studies; it really has been quite an adventure.

I am grateful to Prof. Dr. Reinhard Jahn for always letting a door open for me to his department; it has made a great difference.

I would like to thank the members of my thesis committee for showing full support towards my projects and my ideas.

It goes without saying that I an most thankful to the administration team at the Göttingen Graduate School for Neurosciences and Molecular Biosciences (GGNB) and in particular Kirsten Poehkler, the commitment showed by the GGNB team for the well-being of the students is humbling.

I would also like to thank Dr. Vladimir Plena, who has guided me in the world of Crystallography and has never let me leave his office without a scientific advice or a word of encouragement, as those are so well needed in Crystallography.

I take the opportunity to thank my friend Dr. Stuart Laurence for his support during my time in Göttingen.

I want to also thank Dr. Piotr Neumann for his enthusiasm and for sharing his knowledge and skills, it was truly impressive. 


\section{Table of Contents}

Abstract ..............................................................................................................

1 Introduction ......................................................................................................

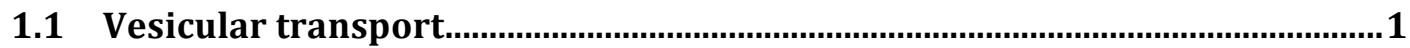

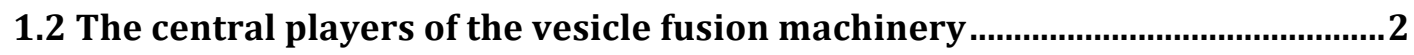

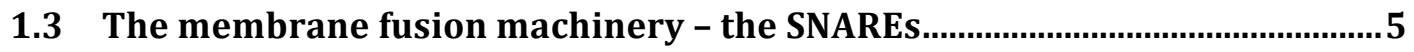

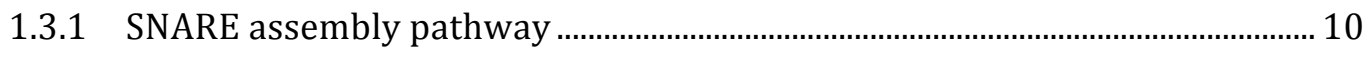

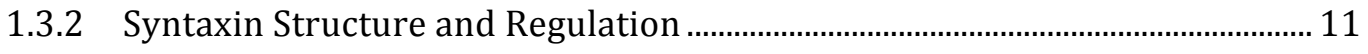

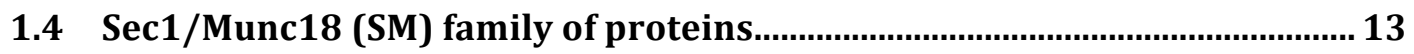

1.4.1 Molecular role of SM proteins: binding to the closed syntaxin or the assembled SNARE complex ............................................................................................... 16

1.4.2 A common mechanism? ...................................................................................... 19

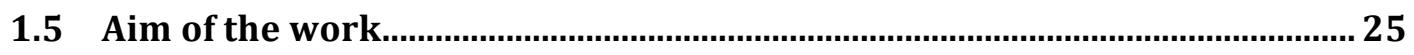

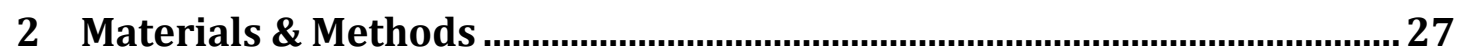

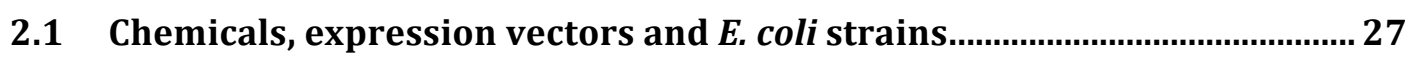

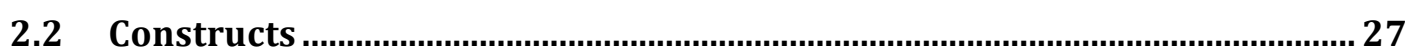

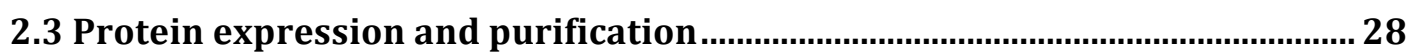

2.4 Determination of protein concentration....................................................... 30

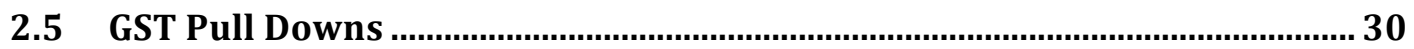

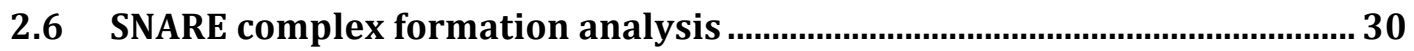

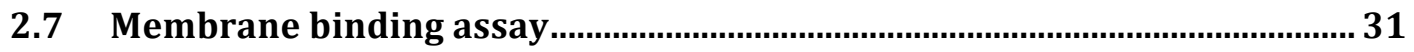

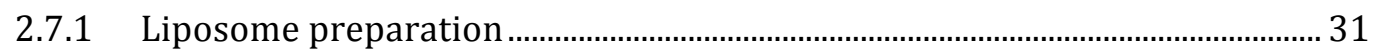

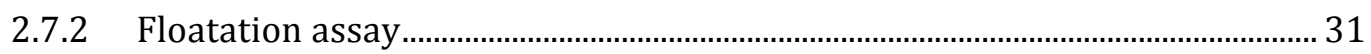

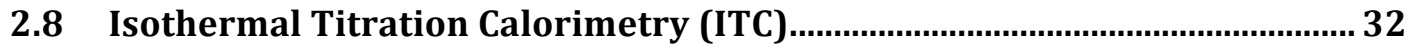

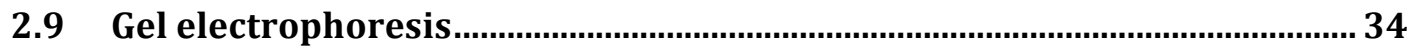

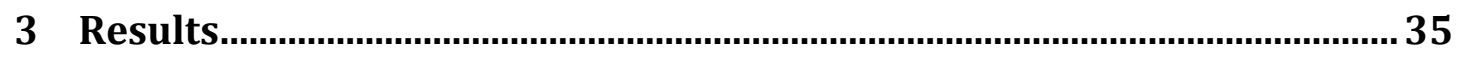

3.1 Characterization of the C. elegance Unc18 interaction to Unc64 ................35

3.1.1 Unc18 binds to Unc64 with high affinity and requires the N-peptide........... 36

3.1.2 Truncation of the SNARE motif severely impairs the binding of Unc64 to Unc18 38

3.2 Mapping of the areas of interaction in Unc64 by Radical Foot-printing in combination with Mass-spectrometry analysis . 
3.3 Peptide 15 reveals a highly conserved region adjacent to layer - 7 and makes a physical interaction with the linker helix 45

3.4 Study of the Sec1p interaction to the Qa SNARE Sso1p and the yeast SNARE complex. 48

3.5 Sec1p does not bind to the Qa SNARE Sso1p, the binary Sso/Sec9 complex nor the ternary SNARE complex in vitro 49

3.6 Sec1p contains a positively charged region on its C-terminal region that can assist Sec1p to localize to the membrane.

3.7 Study of the Sec1p interaction to Sso1p and the yeast SNARE complex in the presence of a lipid membrane 53

3.8 Engineering of a Sec1p construct with increased solubility 54

4 Discussion. 60

4.1 Biochemical characterization of the interaction between Unc18 / Unc6462 4.2 Mapping of interacting areas in Unc18 and Unc64 by mass spectrometry using hydroxyl radical footprinting. Error! Bookmark not defined. 4.3 Sec1p binding to the components of the SNARE complex 68

4.3.1 Pull down studies show no interaction between Sec1p and the SNARE complex......

4.3.2 Sec1p does not bind to the SNARE complex in the presence of a membrane 71

4.4 Mso1p is an obligatory partner necessary for full Sec1p function 73

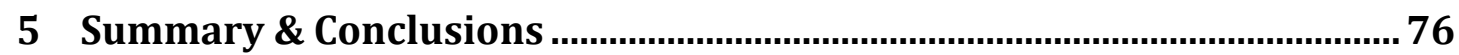

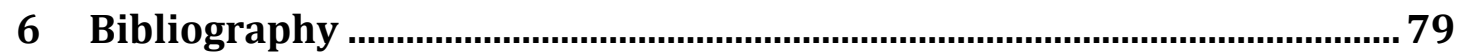

7 Appendix 


\section{List of Figures}

Figure 1 The discovery of the membrane fusion machinery in chronological order

Figure 2 Intermediate stages of SNARE mediated fusion

Figure 3 Four-helix bundle of the SNARE complex representing the classification of the components

Figure 4 Schematic view of the "Open" and "Closed" conformation of Syntaxin(Qa-SNAREs)

Figure 5 Crystal structure of Sso1p, Syntaxin1a and Syntaxin (Monosiga Brevicollis)

Figure 6 Overview of the location in which the different kinds of SNAREs promote fusion

Figure 7 Schematic diagram of Unc64 constructs used in the interaction studies to Unc18

Figure 8 Calorimetric titration of Unc64 (L9A) with wild type Unc18

Figure 9 Calorimetric titration of truncated version of Unc64 with wild type Unc18

Figure 10 Calorimetric titration of Unc64 (I234A) and Unc64 (LE) with wild type Unc18

Figure 11 Sequence coverage of Unc18 and Unc64

Figure 12 Proposed interaction of Unc18 and Unc64 based on the modification found in peptide 4 
Figure 13 Modified regions on the Unc64 sequence upon complex formation

Figure 14 Peptide 15 reveals the dynamics of a region near a loop shown to be important in SNARE complex regulation

Figure 15 Peptide 15 reveals a conserved region near a loop that connects the SNARE motif with Hc-helix

Figure 16 Schematic diagram of the constructs used in the Sec1p interaction studies

Figure 17 Interaction studies of Sec1p to the SNARE complex components

Figure 18 Effect of Sec1p on SNARE complex formation

Figure 19 Sec1p contains a potential membrane binding region

Figure 20 The interaction of Sec1p to the SNARE complex in the presence of a membrane

Figure 21 Sec1p/Munc18a alignment

Figure 22 Superimposition of all the SM proteins

Figure 23 Novel constructs to study the binding of Sec1p to the SNARE components

Figure 24 The loop that connects the Hc-helix with the SNARE domain 


\section{List of tables}

Table 1 Summary of protein constructs used in the studies, expression vectors and purification methods

Table 2 SDS-PAGE recipe

Table 3 Summary of the thermodynamic parameters obtained from all the ITC experiments performed

Table 4 Summary of residue modification on Unc64 upon complex formation 


\section{Abstract}

The members of the Sec1p/Munc18 (SM) family of proteins are indispensible regulatory elements that act at the post-docking step prior to fusion; they exert their function mainly through their interaction to the Qa-SNAREs (Syntaxins). Although studies have shown a high degree of structural homology, different binding modes have been proposed. In the case of the neuronal Munc18-1, an affinity towards a "close conformation" formed by Syntaxin has been reported, while other members of the SM family of proteins seem to interact with the outmost $\mathrm{N}$-terminal region of the syntaxin commonly referred to as the $\mathrm{N}$ peptide. In addition, the baker's yeast Saccharomyces cerevisiae show an affinity towards the assembled SNARE complex. To complicate things further, recent studies have reported that SM proteins might require both the N-peptide and the "close conformation" to interact. Biochemical studies on Unc18, the Munc18 homolog in the nematode Caenorhabditis elegans, have shown that it requires only the N-peptide region of Unc64 to regulate secretion. While in yeast the secretory SM protein Sec1p has been reported not to be able to bind to its cognate Syntaxin Sso1 alone, but only to the already assembled SNARE complex containingg Sso1p, Sec9p (SNAP-25), and Snc1/2p (Synaptobrevin). As these studies use semiquantitative protein-binding assays, I investigated these interactions in more detail using biophysical tools such as Isothermal Titration Calorimetry (ITC). My findings demonstrate that Unc18, just like its mammalian homolog, require both the N-peptide and closed conformation in order to bind with high affinity. Next I evaluated whether Sec1p bind to the SNARE complex in vitro. This was challenging because Sec1 could not be produced in high enough amounts by recombinant means. However, from these studies I conclude that Sec1p does not bind to the SNARE complex when the interaction is studied by means of neither Pull-downs nor when the components are let to assemble in solution, or in the presence of the membrane. In addition, I was able to engineer 
several new Sec1 constructs that in the future can be used to do experiments with techniques that require high concentrations of protein but in exchange will give more detailed insights, ITC is the next goal and Crystallography the ultimate. The new set of constructs seem to be able to make this possible as they show an ability to overcome the solubility barrier that have made it impossible to produce, and as a consequence made it hard to dissect the interaction of Sec1p in vitro. 


\section{Abbreviations}

Cal Calorie

DTT Dithiothreitol

Fig. $\quad$ Figure

GST Glutathion S-Transferase

ITC Isothermal Titration Calorimetry

mM Millimolar

Kd Dissociation constant

PMSF Phenylmethylsulphonylfluoride

$\mathrm{v} / \mathrm{v} \quad$ Volume/volume 


\section{Introduction}

\section{$1.1 \quad$ Vesicular transport}

The eukaryotic cell contains various different membrane-enclosed compartments that perform different tasks that are often vital for the survival of the cell. To fulfill its function each compartment, or organelle, contains its own set of enzymes. Transport between most compartments takes place by vesicular transport: vesicles, tiny sac-like structures, bud off the donor compartment, are then transported along cytoskeletal tracks and upon reaching the target compartment the vesicle membrane and releases its content. Transport vesicles have the ability to move both membrane components and soluble molecules across the cell without them ever have to go through the task of crossing a membrane on the way until they reach the cargos final destination (Bonifacino and Glick 2004). New proteins and lipids are synthesized in the Endoplasmatic Reticulum (ER), transported to the Golgi apparatus. Along this pathway various post-translational protein modifications like glycosylation take place. Upon reaching the trans-Golgi network the material is sorted and targeted into different transport carriers according to their final destination. Vesicles on route to the plasma membrane release their content to the extracellular space, a process called secretion. Cells can also take up material from the outside by endocytosis and phagocytosis. The material is packed into vesicles and transported to internal organelles. All these events require specialized machinery that is capable of fulfilling the tasks with high accuracy and at the same time preserve the integrity of the internal architecture. Each step in the vesicle transport process is carried out by specific protein machineries. The key proteins involved in the different steps of vesicle transport have been identified in the past two decades and found to belong to structurally conserved protein families. 
The life cycle of a transport vesicle begins with the budding at the "donor" organelle. The site is defined by a set of proteins that are responsible for selecting the cargo that is to be loaded into the lumen or incorporated into the membrane of the vesicle. The selection of the cargo is carried by a set of proteins that recognize a specific tag in the proteins sequence. The very same proteins are also responsible for the bending of the membrane and compose the coat that covers the surface of the vesicle. So far, three different types of coated vesicles have been studied extensively and the vesicles they coat depend on the kind of pathway they are involved in. The first kind of vesicles that was studied were the clathrin-coated vesicles (Roth and Porter 1964; Pearse 1975) that are involved in the formation of vesicles that bud from the trans-Golgi network and are further transported to the plasma membrane or the early endosome. Clathrincoated vesicles are also involved in endocytosis. The other two kinds of coats are COPI, involved in intra golgi transport and in retrograde transport between the Golgi to the ER (Letourneur, Gaynor et al. 1994) and COPII vesicles budding from the ER destined to reach the Golgi (Barlowe, Orci et al. 1994). Once the vesicle has been formed, loaded with the specific cargo, and budded off the donor membrane the vesicle is moved by motor proteins along the cytoskeleton (Doussau and Augustine 2000). Upon reaching its final destination, it is tethered/docked to the acceptor membrane and finally fusion is catalyzed. The key proteins involved in the last steps belong to the following protein families: SNAREs, SNARE disassembly proteins, Rab proteins, SM proteins, and a group of tethering factors termed CATCH proteins.

\subsection{The central players of the vesicle fusion machinery}

Our current understanding of the vesicular transport mechanism is the result of decades of detailed studies that have cast light onto the different components that are essential for its function. The existence of intracellular organelles and vesicle trafficking had been discovered in the 1960's (Palade 1975). However, the first systematic studies to uncover the crucial components of this hallmark of the eukaryotic cell were not performed until late 1970's. A 
decisive genetic screen was carried out by Schekman and his colleagues. They used baker's yeast, Saccharomyces cereviseae, as a model organism to perform a genome-wide screen. The screen revealed a series of genes that while mutated exhibited a temperature dependent defect in secretion. These so-called "Secmutants" were divided into 23 complementation groups, each corresponding to a different gene (Novick, Field et al. 1980). Although these gene defects had all in common an impaired secretion, the defect manifested itself in various morphological ways when the cells were inspected by Electron Microscopy (TEM) at non-permissive temperature, and reflected the particular pathway in which they are involved in and at which stage the vesicular pathway is blocked (Novick, Field et al. 1980). In the following years, the function of first 23 SEC genes was brought to light, revealing that they had stumbled over a broad range of factors involved in various aspects of the vesicle transport.

At first, the gene to be studied in more detail by biochemical means was SEC18 whose inactivation lead to the block of the transport of secretory proteins between the ER and the Golgi (Eakle, Bernstein et al. 1988). Its gene product was found to be a cytosolic protein and it was thought that it may bind transiently to small transport vesicles such as those shuttling between ER and the Golgi complex. It was not clear, however, whether it was involved in the budding and loading of vesicles or in fusion.

A few years after these achievements, William Balch and James Rothman employed a cell-free assay to study the secretory pathway of mammalian cells (Balch, Dunphy et al. 1984). Their assay was based on the well-known fact that many proteins, among other modifications, are N-glycosylated during synthesis in the ER. Various modifications of the sugar-side chain take place during its transport through the Golgi stacks. One of the last modifications of the sugar side chain is catalyzed by the enzyme $\mathrm{N}$-acetylglucosamine (GlnNAc) transferase. Balch and Rothman infected CHO cells with the vesicular stomatitis virus (VSV) and isolated Golgi fractions. A glycoprotein encoded by the virus is $\mathrm{N}$ glycosylated in the ER. For their transport assay the researches made use of a CHO line that lacked the key Golgi enzyme GlnNAc transferase activity. The 
isolated Golgi fractions of mutant CHO cells contained radioactive labeled glycoprotein that did not hold N-acetylglucosamine. However, when they mixed those fractions with isolated Golgi from wild-type cells, a source that is capable of modifying the viral protein, they discovered that $\mathrm{N}$-acetylglucosamine was incorporated into the glycoprotein, demonstrating that vesicle transport between the Golgi fractions from different cell lines must have occurred. They also found that transport depended on the presence of ATP and cytosol.

In the following years, the research group headed by James Rothman uncovered and characterized the key cytosolic proteins essential for vesicle fusion. The first protein was found by mild treatment of the cytosol with $\mathrm{N}$ ethylmaleimide (NEM) (Glick and Rothman 1987), a treatment that selectively blocked vesicle transport in the assay. The factor was termed N-ethylmaleimidesensitive factor (NSF). It is a soluble ATPase. Interestingly, it turned out to be homologous to one of the Sec mutants discovered in the genetic screen by Novick and Schekman, Sec18, giving the first clue that yeast and man share a conserved machinery (Wilson, Wilcox et al. 1989) (Griff, Schekman et al. 1992). Rothman and colleagues then found that NSF requires additional factors to carry out its essential role in vesicle transport. First, they discovered a small family of soluble NSF attachment proteins $(\alpha-, \beta-$, and $\gamma$-SNAP). Its yeast homolog is Sec17. Eventually, they discovered that NSF together with SNAP binds to membrane bound SNARE proteins (SNAP receptors). In the presence of the nonhydrolysable ATP analog ATP- $\gamma$ S, NSF and SNAPs formed a large, 20S protein complex with SNARE proteins present in crude detergent extract of brain membranes. They identified SNARE proteins as syntaxin 1 (Bennett, Calakos et al. 1992), SNAP-25 (Oyler, Higgins et al. 1989) and VAMP-2/synaptobrevin 2 (Trimble, Cowan et al. 1988) (Trimble, Cowan et al. 1988; Baumert, Maycox et al. 1989). They also found that NSF promotes the disassembly of the 20 S complex.

At that time, synaptobrevin 2 had been established already as a major membrane protein of synaptic vesicles, whereas the other two proteins were known to be located primarily in the presynaptic plasma membrane. All three 
proteins were also found to be cleaved by different clostridial neurotoxins, which act as very specific proteases. In addition, it was found that the three SNARE proteins formed a very stable, SDS-resistant ternary complex. Thus, it became clear that SNARE proteins formed a tight complex between vesicle and target membrane. An account of those exciting discoveries from the perspective at that time is given in (Barinaga 1993) and (Ferro-Novick and Jahn 1994) and from a more present point of view in Scheller and Jahn (Jahn and Scheller 2006) (Fig. 1).

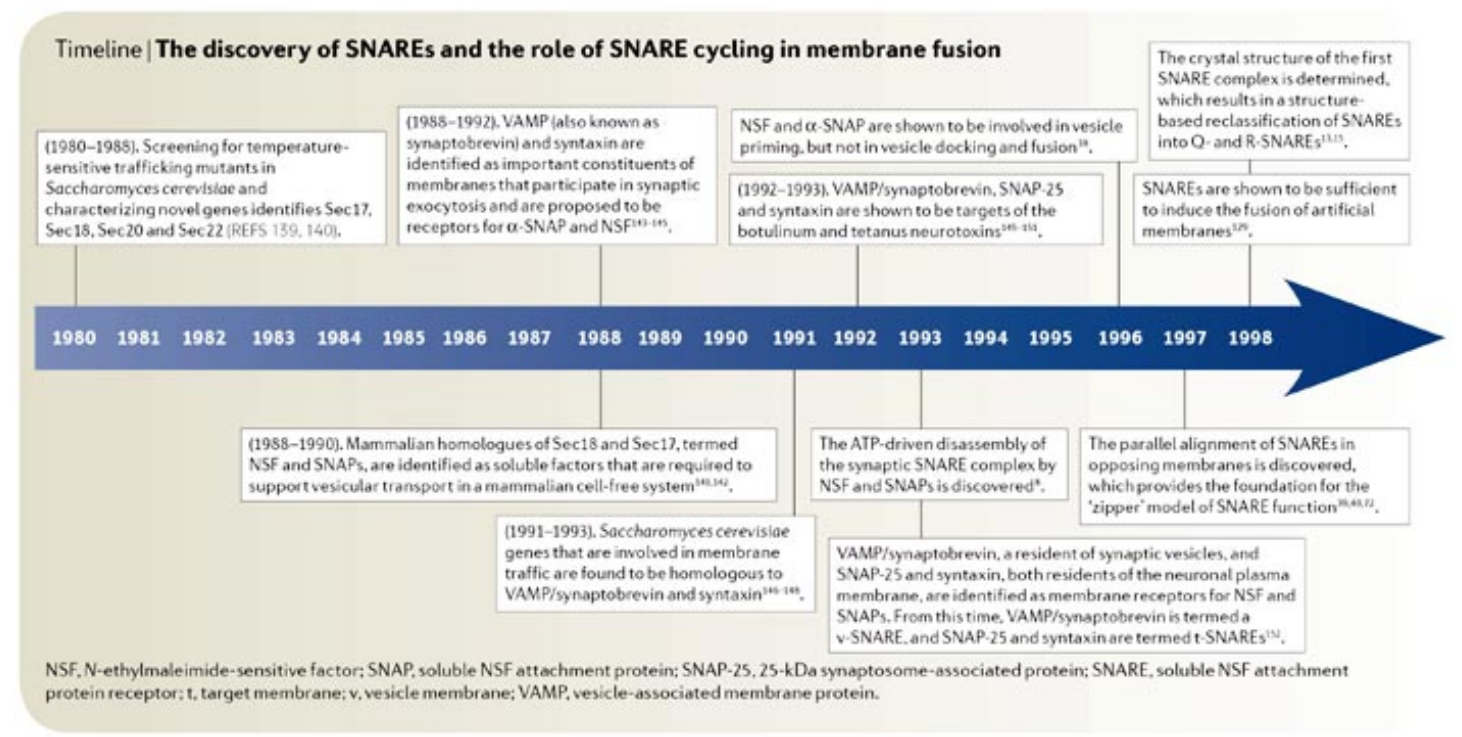

Figure 1. The discovery of the membrane fusion machinery in chronological order. (Picture is taken from Jahn R. and Scheller R. H., 2006)

\subsection{The membrane fusion machinery - the SNAREs}

Originally, it was thought that the action of NSF and SNAP promotes the fusion of vesicle and target membrane (Rothman 1994), whereas the SNARE complex between vesicle and target membrane formed in an antiparallel fashion and thereby only led to vesicle docking but not fusion. The discovery that the three SNARE proteins are aligned in parallel in the SNARE complex (Hanson, Roth et al. 1997), however, was a decisive turning point in our conceptual understanding of the vesicle fusion machinery, as this orientation could not be reconciled with a static docking role. Instead, it was put forward that SNAREs 
might assemble in a zipper-like fashion between the two fusing membranes. This zipper-like assembly process alone, without energetic input by other factors, so the idea would be sufficient to drive membrane fusion (Hanson, Roth et al. 1997). This idea was readily tested and proven to be correct by Rothman and colleagues, who were able to monitor fusion between artificial membranes containing only SNARE proteins (Weber, Zemelman et al. 1998). For this they inserted synaptobrevin into one population of liposomes and co-reconstituted syntaxin and SNAP-25 into the other population. Fusion was monitored by a lipid dequenching assay that had been used before to investigate fusion of enveloped virus with host cells. With the discovery and functional characterization of the neuronal SNARE proteins it became clear these proteins belong to a larger family of proteins. For example, the screen carried out by Novick and Schekman had found already three different SNARE proteins, Sec9, Sec20, and Sec22. Different SNARE proteins are found on membranes throughout the exocytotic and endocytotic pathways. In fact, they all are believed to provide the necessary force to overcome the repulsive electrostatic barrier created by the negative net charge of cellular membranes in all vesicle trafficking steps (Hanson, Roth et al. 1997). Although SNAREs are found at different sites throughout the cell at their specialized sites where they exert their individual function, they all have one shared characteristic in the form of a domain commonly referred to as the "SNARE motif". This motif is composed of a coiled-coil heptad repeat of amino acids of roughly 60-70 residues in length (Fasshauer, Eliason et al. 1998) (Sutton, Fasshauer et al. 1998). All SNARE proteins are thought to assemble into an extended four-helix bundle (Sutton, Fasshauer et al. 1998; Stein, Weber et al. 2009) between the fusing membranes.

The SNARE complex can be composed of three proteins only as in the case of the neuronal SNAREs, as one of the SNAREs, SNAP-25, contributes two helices to the complex. The same architecture was found for the yeast secretory SNARE complex consisting of Sso1/2 (i.e. the syntaxin homolog), Snc1/2 (i.e. the synaptobrevin homolog), and Sec9 (i.e. the SNAP-25 homolog). Most other SNARE sets, however, are composed of four different SNARE proteins. For example, the crystal structures and biophysical properties of SNARE complexes 
involved in early (Zwilling, Cypionka et al. 2007) and late endosomal fusion (Antonin, Holroyd et al. 2000) are very similar to the one of the neuronal SNARE complex (Sutton, Fasshauer et al. 1998) and the one involved in yeast secretion.

It is compulsory to have at least one SNARE protein anchored by a transmembrane region in each membrane destined to fuse. When in close enough proximity the SNARE components recognize and bind to one another through their motifs and form a four helix bundle that will bring the membranes together to the point that lipid mixing can take place leading ultimately to fusion (Fig. 2) (Sutton, Fasshauer et al. 1998), (Antonin, Fasshauer et al. 2002), (Zwilling, Cypionka et al. 2007), (Strop, Kaiser et al. 2008). How SNAREs provide the mechanical force to make the fusion takes place is still in debate and although it has been proposed that the complex formation releases enough energy to overcome the repulsive barrier and physically merge them (Hanson, Roth et al. 1997), (Lin and Scheller 1997), this might not be the case as when dissecting the energetics of the reaction show that there is not enough energy to overcome the calculated value that fusion requires (Wiederhold and Fasshauer 2009). Our understanding of how SNAREs interact and catalyze the fusion reaction has been and still is at the center of many studies. The results obtained have revealed many details on the mechanism in which they interact and form the SNARE complex which seems to take place in a zippering-like way starting at the N-terminal region and propagating along the SNARE motif reaching the Cterminal region proximal to the membrane (Hanson, Roth et al. 1997), (Fasshauer, Sutton et al. 1998), (Fiebig, Rice et al. 1999) (Foran, Shone et al. 1994). 

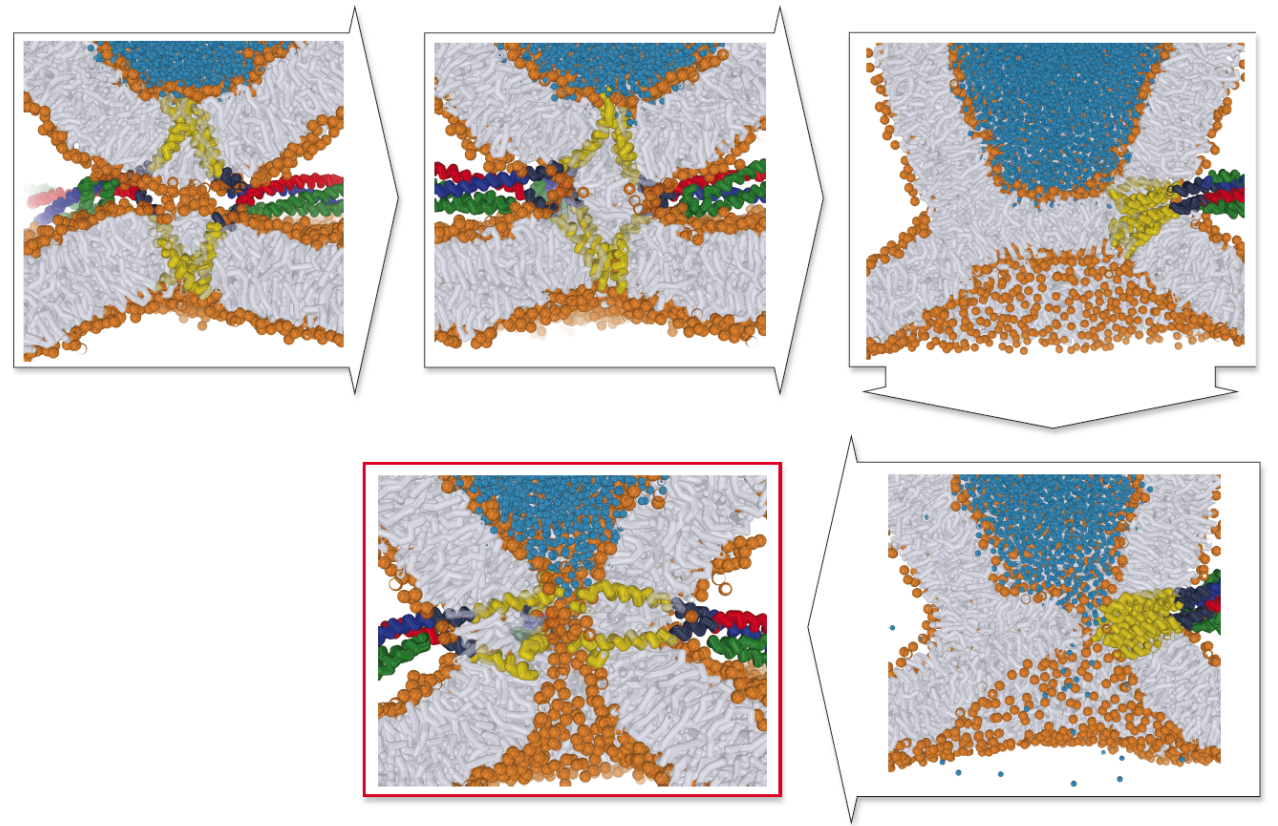

Figure 2. Intermediate stages of SNARE mediated membrane fusion.

Different snapshots of a simulated sequence of events in which the membrane anchored SNARE proteins pull the membranes into close proximity in order for the lipids to start mixing, this will ultimately lead to merging of the membranes (Sutton, Fasshauer et al. 1998), (Antonin, Fasshauer et al. 2002), (Zwilling, Cypionka et al. 2007), (Strop, Kaiser et al. 2008). (Picture is taken from Jahn R. and Fasshauer D., 2012)

After SNARE proteins had been discovered, more and more studies kept uncovering additional members of the SNARE family. At that point a first classification system was established which was mainly based on the cellular location of SNARE proteins and the topology of the complexes believed to be formed between transport vesicle and target membrane. The SNAREs were simply divided into either v-SNAREs (i.e. vesicle SNARE) found on the cargo vesicle and t-SNAREs (i.e. target SNARE) found on the target membrane with which the transport vesicle will fuse (Söllner, Bennett et al. 1993). This v-tSNARE classification system worked reasonably well for describing the formation of most SNARE complexes, but has problems as it is not well suited to describe homotypic fusion events during which membranes of the same compartment fuse, for example during vacuole fusion (Wickner and Haas 2000). Another problem is encountered when SNARE proteins are involved in two different trafficking steps, as is the case for Sec22p found to work in yeast's anterograde as well as the retrograde pathway taking place in-between the ER and the Golgi (Dilcher, Veith et al. 2003), (McNew, Parlati et al. 2000), (Lewis, 
Rayner et al. 1997), (Burri, Varlamov et al. 2003). Sec22 is therefore sometimes referred as both a t-SNARE as well as a v-SNARE. After the structure of the SNARE complex was solved, it was possible to classify SNARE proteins accordingly. Most striking was the strict conservation of the central layer of the extended coiled coil structure of the SNARE complex. The four subunits of the complex form 16 stacked hydrophobic residues or layers, except for the central layer that is referred to as the " 0 " layer. This layer is generally composed of a set of hydrophilic residues: a glutamine (Q) residue contributed by syntaxin, two glutamines $(\mathrm{Q})$ by the two SNAP-25 helices, and one arginine (R) by synaptobrevin. Accordingly, SNAREs were classified into Q- and R-SNAREs. A more detailed analysis of the structure allowed for a finer classification into four main groups (Qa-, Qb-, Qc, and R-SNAREs) reflecting their position in the fourhelix bundle (Fig. 3) (Fasshauer, Sutton et al. 1998) based on the structure of the core SNARE complex. This classification system was further refined by an elaborate phylogenetic approach, which confirmed the four main groups but at the same time revealed 20 subclasses of SNAREs proteins that probably represent the repertoire of the common ancestor of all eukaryotic cells. These 20 subclasses of SNAREs probably assemble into four to five principal SNARE complexes that participate in the major trafficking steps of the cell (Kloepper, Kienle et al. 2007). The SNARE proteins involved in secretion belong to group IV.

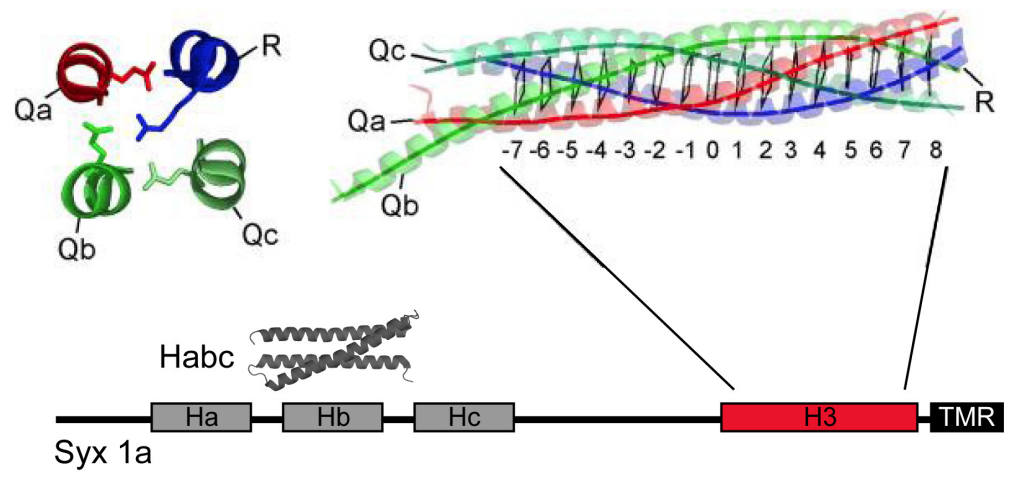

Figure 3. Four-helix bundle of the SNARE complex representing the classification of the components. This a representation of the distinct layers in which the complex is divided, every layer is marked by a conserved hydrophobic residue except for layer " 0 " which contains a glutamine in the case of Syntaxin $(\mathrm{Q} a)$, SNAP-25 (Qb and Qc), while the Synaptobrevin contains an arginine (R-SNARE) (Fasshauer, Sutton et al. 1998). 


\subsubsection{SNARE assembly pathway}

It has been shown by different methods that SNARE motifs are mostly unstructured when unpaired in solution but until the moment in which they interact with each other and form the helical four helix (Fasshauer, Bruns et al. 1997) (Fiebig, Rice et al. 1999), (Fasshauer, Otto et al. 1997) (Hazzard, Sudhof et al. 1999). It was also found that SNARE complex formation is relatively slow in vitro. These properties suggest that SNARE complex assembly between two membranes must be regulated and guided by additional factors in the cell. In other words, the formation of the SNARE complex is not a single event in which all the components simply collide. For secretory SNAREs it is generally believed that the two plasma membrane SNAREs, syntaxin and SNAP-25 in animals or Sso1/2 and Sec9 in yeast, interact first and provide a binding site for the vesicular R-SNARE synaptobrevin in animals or Snc1/2 in yeast. Indeed, a pronounced hysteresis in the unfolding and folding (i.e. assembly) transition of SNARE complexes indicates that assembly occurs via a less stable intermediate, very likely composed of a complex formed between the two Q-SNAREs in the plasma membrane (Fasshauer, Antonin et al. 2002).

In vitro studies on neuronal SNAREs showed, however, that syntaxin 1 and SNAP-25 readily form a complex with a stoichiometry of 2:1 (Fasshauer, Otto et al. 1997). Very probably in this complex the binding site of synaptobrevin is blocked by a second syntaxin molecule, slowing the formation of the ternary SNARE complex. One way avoid the proteins from falling into the off-pathway is by the pre-formation of the complex with an excess of SNAP-25 (Fasshauer and Margittai 2004) or by adding an engineered C-terminal synaptobrevin fragment (Melia, Weber et al. 2002; Pobbati, Stein et al. 2006). The major advantage of this approach is that the fragment prevents a second syntaxin from binding but still leaves the synaptobrevin binding site accessible (Pobbati, Stein et al. 2006). This engineered complexes, widely referred to as the "Delta N-Complex", is an alternative tool to study SNARE complex formation that is otherwise too slow 
due to the rate limiting step of forming a binary acceptor complex between Syntaxin 1a and SNAP-25, its short lifetime and the always present possibility of taking an off-path route (Fasshauer and Margittai 2004). As mentioned above, the homologous yeast secretory SNAREs Sso1 and Sec9 form an equivalent acceptor complex for the vesicle SNARE Snc1. Interestingly, the Sso1/Sec9 complex was found to adopt a 1:1 configuration. Still, even when the Sso1/Sec9 complex is preformed binding of Snc1 is relatively slow (Nicholson, Munson et al. 1998), suggesting that a complete understanding of the assembly of the core SNARE complex is not achieved yet. Thus, despite their seeming simplicity, the conformational dynamics and the assembly pathway of SNARE proteins is extraordinarily complex and not yet fully understood. Much less is known of the assembly pathway of SNARE complexes involved in other trafficking steps in the cell.

\subsubsection{Syntaxin Structure and Regulation}

Of the three SNARE proteins involved in secretion only one, the QaSNARE, contains a larger additional domain. This domain forms a three-helix bundle structure and is referred to as Habc domain (Fernandez, Ubach et al. 1998; Lerman, Robblee et al. 2000) (Bracher and Weissenhorn 2004)). Sometimes it is also referred to as N-terminal regulatory domain in order to indicate its putative role. This has been studied most intensively for syntaxin 1 (Fernandez, Ubach et al. 1998) (Lerman, Robblee et al. 2000) (Bracher and Weissenhorn 2004) (Misura, Scheller et al. 2000) (Burkhardt, Hattendorf et al. 2008) and the yeast secretory syntaxin Sso1/(Nicholson, Munson et al. 1998; Fiebig, Rice et al. 1999) (Munson, Chen et al. 2000) (Munson and Hughson 2002). Interestingly, this domain arrangement is found in all type of Qa-SNAREs, suggesting that it represents a conserved regulatory feature of all different types of SNARE complexes. For several Qa-SNAREs it has been demonstrated that the SNARE motif can loop back and bind to its own Habc domain (Fig. 4A) (MacDonald, Munson et al.). 
The way in which the Habc domain folds back seems to have no other role than to make the SNARE motif inaccessible for interaction and therefore unable to take part in SNARE complex formation, and in-turn inhibit fusion. Indeed, this conformational switch seems to be a regulatory mechanism. When the Habc domain is bound to its SNARE motif the conformation is referred to as "closed" (Fig. 4A), while the unbound state is referred to be as "open" (Fig. 4B) and can take part in SNARE complex formation (Fig. 4C) (Pevsner, Hsu et al. 1994), (Misura, Scheller et al. 2000), (Nicholson, Munson et al. 1998). What the natural state of syntaxin is in solution has been debated and different conclusions have been reaches from different studies. NMR studies have shown that the neuronal Qa-SNARE syntaxin 1a adopts mostly a close conformation, while single moledule FRET studies showed that $70 \%$ of the Syntaxin $1 \mathrm{a}$ is open and that the two states rapidly switch (Dulubova, Yamaguchi et al. 2001; Margittai, Widengren et al. 2003; Chen, Lu et al. 2008), (Dulubova, Yamaguchi et al. 2002; Yamaguchi, Dulubova et al. 2002). As will be outlined below, syntaxin 1a has been found to be in a tight closed conformation when bound to the protein Munc18a (Fig. 5B \& C).

A

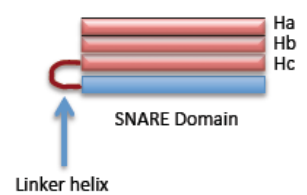

B

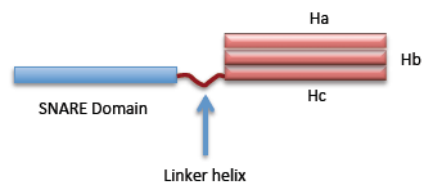

C

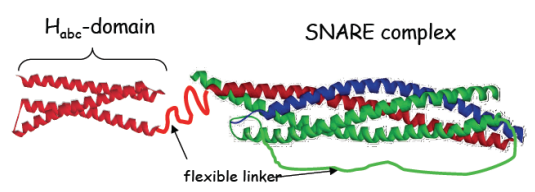

Figure 4. Schematic view of the "Open" and "closed" conformation of syntains (Qa-SNAREs).

The structures of Qa-SNAREs alternate between two functionally different conformations; Syntaxins can obtain an "open" or "close" conformation. The state in which the syntaxin is depends on whether the threehelix domain (represented as the red region in figure A and B) is bound to the SNARE domain (represented as the blue region in figure $\mathrm{A}$ and $\mathrm{B}$ ) or not. The interaction of the Habc domain on the SNARE domain makes it inaccessible for SNARE complex formation as its binding region is covered up (A), Some SM proteins have been reported to have show a preference towards this conformation. On the other hand, the displacement of the Habc domain (B) allows it to interact with the other SNARE components and form the complex (C). The SNARE domain and the Habc domain are connected through a helix loop that attaches the C-terminal region of the Hc helix with the N-terminal region of the SNARE motif, mutations in this loop creates a constantly "open" mutant. 
By contrast, the yeast syntaxin Sso1p has been found to adopt a very tight closed conformation on its own (Fig. 5A). (Nicholson, Munson et al. 1998; Fiebig, Rice et al. 1999) (Munson, Chen et al. 2000) (Munson and Hughson 2002). In fact, individual Sso1 assembles into a SNARE complex about 2000 times slower than its isolated SNARE motif (Nicholson, Munson et al. 1998), whereas syntaxin 1a is merely less than 10 times than its isolated SNARE motif (Margittai and Langen 2004). A closer inspection of the sequences has revealed yet another conserved region that can be of regulatory interest besides the Habc domain: the linker region between the SNARE motif and the Hc helix. This linker can obtain a helical structure (Misura, Scheller et al. 2000; Munson, Chen et al. 2000). Mutations in this region have shown to increase the activity of the syntaxin, apparently shifting it towards a more "open" configuration(Dulubova, Sugita et al. 1999; Munson, Chen et al. 2000).

A

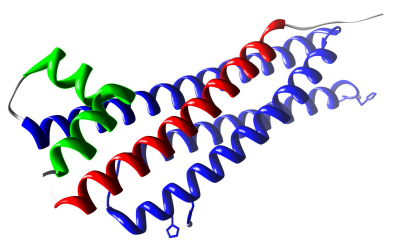

B

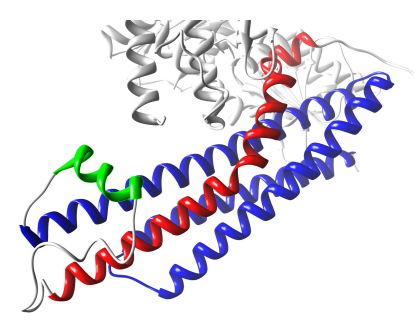

C

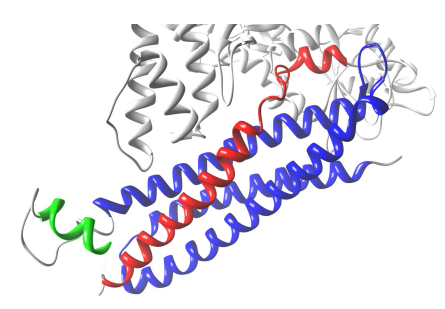

Figure 5. Crystal structures of Sso1p, Syntaxin1a and Syntaxin (Monosiga Brevicollis).

A comparison of the crystal structures of (A) Sso1p (PDB: 1FIO), (B) Syntaxin1a in complex with Munc18-1 (PDB: 3C98) and (C) Syntaxin in complex with Munc18 (PDB: 2XHE) from Monosiga brevicollis. These structures represent the close four-helix bundle promoted by the binding of SM proteins except for in the case of Sso1p that seems to adopt a very stable close conformation on its own.

\subsection{Sec1/Munc18 (SM) family of proteins}

The members of the Sec1p/Munc18 (SM) family of proteins are essential component of the vesicle fusion machinery and exert their function at the postdocking step prior to fusion. Different family members participate in different vesicle trafficking steps. The family is composed of proteins that are soluble, but are also found on the membrane where they work in close collaboration with the 
SNARE proteins, in particular the syntaxins (Qa-SNARE) (Carr and Rizo ; Halachmi and Lev 1996; Rizo and Sudhof 2002; Toonen and Verhage 2003; Toonen and Verhage 2007). Their importance has been reported in numerous studies and their absence is represented by serious defects in vesicular fusion at the particular site at which the particular SM protein is active.

As mentioned above, Sec1 had been found in the screen for yeast mutants defective in secretion (Novick, Field et al. 1980). However, the first more detailed characterization of the gene coding for the yeast SM protein Sec1p, whose inactivation leads to accumulation of secretory vesicles, was carried out later. Until this point, other SM protein members, Sly1 (Ossig, Dascher et al. 1991) (Dascher, Ossig et al. 1991) and Vps33 (Robinson, Klionsky et al. 1988); (Wada, Kitamoto et al. 1990) had been discovered by genetic screens in yeast as well. Another, Vps45, was found later (Cowles, Emr et al. 1994) (Yoshida, Ohya et al. 1995). Then a genetic screen in the fruitfly, Drosophila melanogaster, uncovered Rop, which turned out to be homologous to Sec1, Sly1, and Vps33, establishing their family bonds (Salzberg, Cohen et al. 1993). Rop was found to be expressed only in the central nervous system, but its precise function remained unclear.

At that time, a neuronal SM protein had been found already by Sidney Brenner in his classical screen in the nematode Caenorhabditis elegans. He had selected the worm as a promising animal for investigating the development and function of a simple nervous system. Using chemicals to induce genetic mutations, Brenner had isolated worms with unusual or uncoordinated movements (i.e. UNC, (Brenner 1974)). One of the mutations occurred in the gene UNC-18, which, as it was only established later, codes for the SM protein Unc-18. Incidentally, another mutation uncovered in this screen is the gene UNC64, which encodes for homolog of syntaxin 1. (Ogawa, Harada et al. 1998). In fact, a closer inspection of unc-18 mutant had shown that the severely paralyzed animals accumulated the neurotransmitter acetylcholine although their acetylcholine esterase activity was normal (Hosono, Hekimi et al. 1992). Shortly after Hugh Pelham pointed out that Sec1 and Unc-18 are homologous and 
suggested, based on the similarity of the phenotypes, that both gene products in fusion of secretory vesicles (Sweet and Pelham 1993).

Another breakthrough came when a novel syntaxin 1 binding partner turned out to be the mammalian homolog of Unc18 and Sec1 (Hata, Slaughter et al. 1993) (Garcia, Gatti et al. 1994); (Pevsner, Hsu et al. 1994). The protein was termed Munc18 (mammalian Unc18), rbSec1 (rat brain Sec1) or n-Sec1 (neuronal Sec1). Later the entire family was called SM proteins for Sec1/Munc18. Two other vertebrate homologs of Munc18-1 were found thereafter and termed Munc18-2 (Hata and Sudhof 1995),(Katagiri, Terasaki et al. 1995) and Munc18-3 (Tellam, Macaulay et al. 1997).

Generally, SM proteins are thought to interact with SNARE proteins, probably directly via the Qa-SNARE or syntaxin. Currently, it is thought that the SM protein family consists of four main types that are highly conserved in all eukaryotes and that appear to function in different vesicle trafficking steps within the cell (Carr and Rizo) (Rizo and Sudhof 2002) (Toonen and Verhage 2003) (Toonen and Verhage 2007):

1. Sec1 or Munc18 are involved in secretion and work together with secretory syntaxins (i.e. Qa-SNARE of type IV; for example Sso1 or Sso2 in yeast and syntaxin 1 in regulated secretion).

2. Sly1 is involved in ER-Golgi trafficking and interacts with two different types of Qa-SNAREs: type II (Sed5 or syntaxin 5) and type 1 (Ufe1 or syntaxin 18).

3. Vps45 is involved in trafficking between the trans-Golgi and endosomes and interacts with Qa-SNAREs of type III.a (Tlg2 or syntaxin 16).

4. Vps33 is involved in vacuolar fusion in yeast. It is part of a larger protein complex called HOPS complex. It is not clear yet whether it binds directly to a Qa-SNARE. 


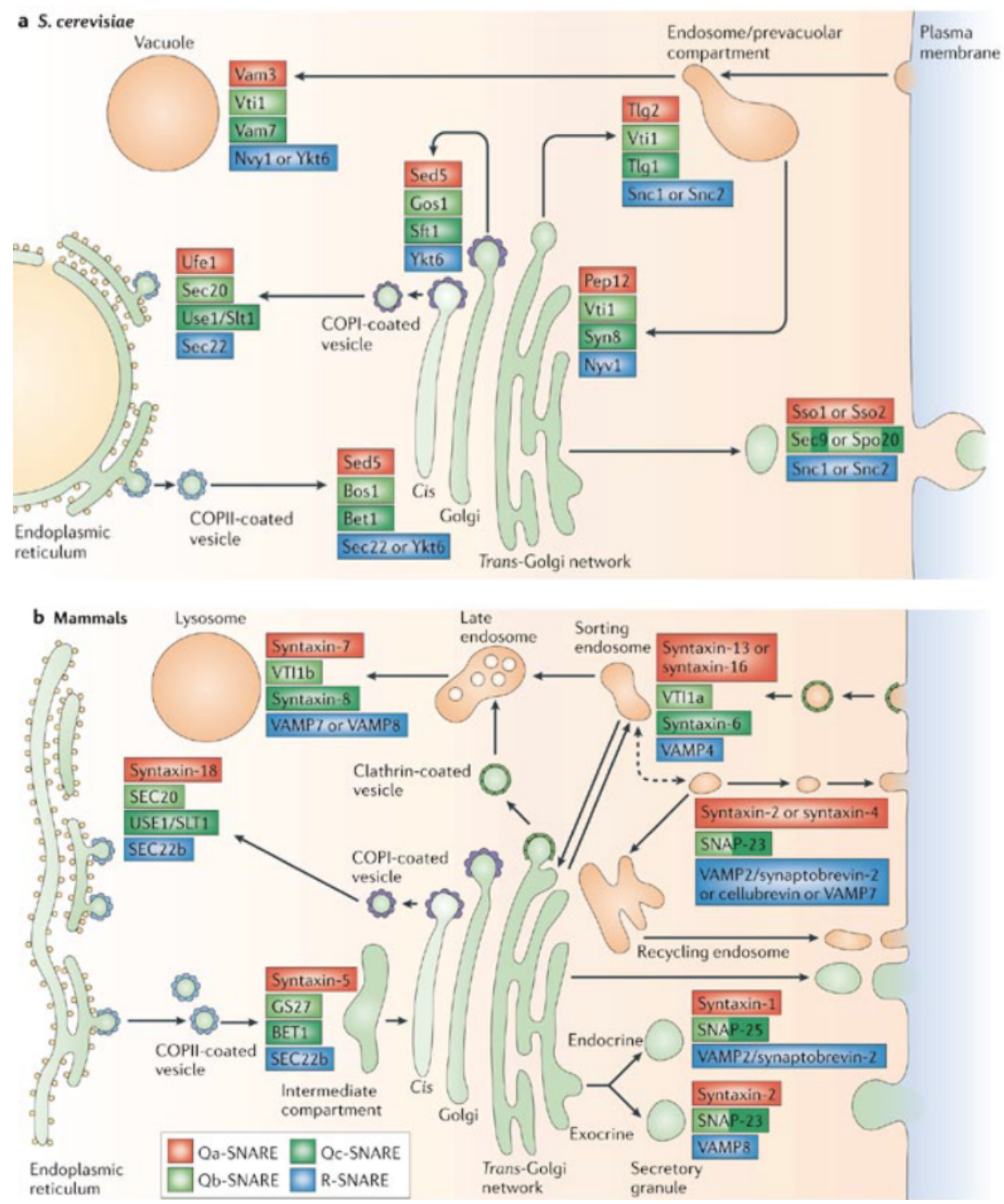

Figure 6. Overview of the location in which the different kinds of SNAREs promote fusion

Overview of different locations in which SNARE act to promote membrane fusion in (A) yeast and (B) mammals. (Picture taken from Jahn R. Scheller R. H., 2006)

\subsubsection{Molecular role of SM proteins: binding to the closed syntaxin or the assembled SNARE complex}

Despite many years of intensive research there is still some controversy over the molecular mechanism of SM proteins (Carr and Rizo ; Halachmi and Lev 1996; Rizo and Sudhof 2002; Toonen and Verhage 2003; Verhage and Toonen 2007; Sudhof and Rothman 2009). Loss-of-function mutations for all different 
SM protein members show comparable phenotypes as they lead to very severe impairment of vesicle docking and fusion. This strongly suggests that all types of SM proteins are essential for the last step in vesicle trafficking. As outlined earlier, the core fusion machinery is thought to consist of SNARE proteins that assemble between vesicle and target membrane, a reaction that eventually leads to membrane merger. The neuronal SM protein Munc18-1 was discovered as a binding partner of syntaxin 1a. In fact, most other SM proteins are also thought to interact with the SNARE machinery, mostly through a direct interaction with the respective syntaxin. The interaction between Munc18-1 and syntaxin 1a was then investigated in more detail. These in vitro studies revealed that the binding of Munc18-1 to syntaxin 1a prevented syntaxin from forming a SNARE complex with its partner SNAREs SNAP-25 and synaptobrevin (Pevsner, Hsu et al. 1994), (Yang, Steegmaier et al. 2000), (Dulubova, Sugita et al. 1999). When the crystal structure of the Munc18-1/syntaxin 1a complex was solved, it was discovered that Munc18-1 arrests syntaxin in a tight closed conformation (Misura, Scheller et al. 2000). Munc18a seems to grasp the entire four helical bundle of syntaxin 1a and using the helices from the Habc domain to cover up the H3 domain (Fig. 5B \& C) (Misura, Scheller et al. 2000) hence, in vitro studies seem to be in strong contradiction with in vivo knock-out studies in which the lack of Munc18 rather blocks secretion instead of promoting a constant and unregulated secretion as it would be expected (Voets, Toonen et al. 2001) (Gulyas-Kovacs, de Wit et al. 2007) (Verhage, Maia et al. 2000). It was therefore speculated that the tight Munc18-1/syntaxin 1 complex represented merely a specialization of the neuronal secretory system. It was speculated that the true activity of Munc18 was to bind to the assembled SNARE complex in order to help this process or to directly participate in membrane fusion (Rizo and Rosenmund 2008) (Sudhof and Rothman 2009) (Shen, Tareste et al. 2007) (Deak, Xu et al. 2009).

Although this idea appears to resolve the problem, it should be noted that the bound syntaxin eventually needs to be handed over to the fusion machinery, but it is unclear how syntaxin can leave the tight grip of Munc18. A direct interaction of Munc18a with the core SNARE complex has indeed been reported (Dulubova, Khvotchev et al. 2007). It should be noted, however, that the affinities 
for the interaction with the assembled SNARE complex are in the micromolar range only (Dulubova, Khvotchev et al. 2007) (Deak, Xu et al. 2009). By contrast, Munc18-1 binds to syntaxin with very high affinity $(\approx 1 \mathrm{nM})$.

The idea that Munc18-1 might bind to the assembled SNARE complex arose when the interaction of the SM protein Sly1 with its cognate syntaxins Sed5 or Ufe1 was studied in more detail. It was found that Sly1 exclusively binds to a very short region of both the Sed5p and Ufe1p N-terminal region rather than to the closed Syntaxin as had been previously observed (Grabowski and Gallwitz 1997) (Yamaguchi, Dulubova et al. 2002). The crystal structure of the Sly1/Sed5 complex demonstrated that the $\mathrm{N}$-terminal region of Sed5 binds to the outer surface of the SM protein, although the structure of Sly1 is very similar to the one of Munc18-1 (Bracher, Kadlec et al. 2002). In this configuration, Sly1 does not lock Sed5 into a closed conformation and thus could help assembling the SNARE complex (Arac, Dulubova et al. 2005) (Peng and Gallwitz 2002) (Peng and Gallwitz 2004). A similar way of interacting with its cognate syntaxin Tlg2p/Syntaxin 16 was discovered for the SM protein Vps45p (Dulubova, Yamaguchi et al. 2002) and for Munc18-3 and syntaxin 4 (Latham, Lopez et al. 2006) (Hu, Latham et al. 2007). As far known, SNARE assembly is also not blocked in these cases. Hence, according to this idea the limited interaction to a small peptide (rather than grasping the four helix bundle) of the syntaxin was thought to allow the binding of the SM protein and at the same time permit the SNARE complex formation by leaving the $\mathrm{H} 3$ domain accessible to interact (Toonen and Verhage 2003) (Sudhof and Rothman 2009) (Carr and Rizo 2010). The short N-terminal stretch of Qa-SNAREs, referred to as N-peptide, contains a conserved motif. The majority of the interactions involve two residues, a highly conserved arginine in a DRT motif and a hydrophobic residue, a phenylalanine in Sed5 and a leucine in syntaxin 4. This hydrophobic residue fits into a hydrophobic pocket of the respective SM protein. 
That notion that SM proteins mainly act on the assembled SNARE complex was also supported by the finding that the secretory yeast SM protein Sec1 was found to interact exclusively with the assembled SNARE complex but not to its cognate syntaxins Sso1 and Sso2 (Carr, Grote et al. 1999). Instead, Sec1p seems to use a different mechanism since Sso1p (the syntaxin homologue in the yeast secretory pathway) does not contain an N-peptide sequence equivalent to the one found in i.e. Syntaxin $1 \mathrm{a}$, the lack of importance in this region has been showed in studies in which Sso1p has been truncated all the way to residue number 31 (Togneri, Cheng et al. 2006). Intriguingly, through a secretion screen and the extensive use of yeast genetics and pull-downs an obligatory binding partner first discovered as a suppressor of sec1-1 mutation was found (Aalto, Jantti et al. 1997). This suppressor has been reported to be a soluble protein of $17 \mathrm{KDa}$ found enriched in the microsomal membrane fraction but also contains the interesting property of having a pI of about 10 . The presence of Mso1p is essential for secretion to take place as it has been shown by knock out and the resulting phenotype characteristic of accumulation of secretion vesicles resembles the one previously observed in Sec1p deletion, clearly showing that it is involved in the terminal stage of secretion. Moreover, disruption of the Mso1p gene is lethal in strains that also contain mutations in the Sec1, Sec2 and Sec4 genes (Aalto, Jantti et al. 1997).

\subsubsection{A common mechanism?}

Recently, new light has been shed on the question of whether SM proteins share a common mechanism and whether Munc18-1 is able to interact with the $\mathrm{N}$-terminal of syntaxin $1 \mathrm{a}$. In the revised crystal structure of the $\mathrm{N}$-peptide is bound to the same binding pocket, while the remainder of Syntaxin 1a is bound in the central cavity of Munc18-1 (Burkhardt, Hattendorf et al. 2008). A similar binding mode was found for a Munc18/syntaxin 1a complex from the choanoflagellate Monosiga brevicollis (Burkhardt, Stegmann et al.), a unicellular relative of metazoans. Moreover, biochemical data suggest that Vps45 and 
syntaxin 16 (Burkhardt, Hattendorf et al. 2008) or Tlg2 (Furgason, MacDonald et al. 2009) make use of both binding sites as well. Apparently, SMprotein/syntaxin complexes generally involve two spatially distinct binding sites but their relative affinities vary widely between different complexes. Interestingly, the two binding sites appear to act together in controlling SNARE complex formation as deletion of the N-peptide activates the SNARE motif of syntaxin although the affinity of the complex is hardly changed (Burkhardt, Hattendorf et al. 2008). Apparently, full "opening" of syntaxin is not required for SNARE binding. This view is supported by recent data obtained with the LE mutant (L165A/E166A) of syntaxin that was originally considered to be constitutively open. Despite some initial controversies (Dulubova, Yamaguchi et al. 2003), it is now known that LE-syntaxin interacts with Munc18 with high affinity using both sites, i.e. it is at least partially closed in this complex. However, SNARE complex formation is not prevented by when the LE mutant is bound by Munc18 (Ma, Li et al. ; Burkhardt, Hattendorf et al. 2008).

Taken together, this suggests that binding of Munc18a to both the closed conformation and to the N-peptide of syntaxin $1 \mathrm{a}$ is an integral part of the reaction cascade during which Munc18-1 guides syntaxin towards productive SNARE complex formation which, at least at the onset, may not require major conformational changes. An inspection of the crystal structure of the Munc18/syntaxin complex shows that already a small rearrangement of the Nterminal part of the SNARE motif of syntaxin that is shielded by a short helix in the linker region in the closed conformation, might be sufficient to allow for binding of SNAP-25. It needs to be mentioned that this view, at least at a first glance, is difficult to reconcile with a recent study using gene replacement in $C$. elegans. Here, syntaxin was replaced by two separate recombinant proteins, one containing the membrane anchor and the SNARE motif, and a second containing the N-terminal region (Habc including the N-terminus) fused to a separate transmembrane domain. Partial rescue of neurotransmission was observed but only when both proteins were expressed (Rathore, Bend et al.), thus supporting the recruitment model described above. However, even when expressed as separate entities the two domains of syntaxin can bind to Munc18 in a tripartite 
complex (Burkhardt, Hattendorf et al. 2008), and it is thus conceivable that the tripartite complex can hand over the SNARE motif of syntaxin to its partner SNAREs.

Despite such progress, it is still unclear how the interaction between syntaxin and Munc18 ultimately promotes SNARE-zippering. Recent experiments in which the effect of Munc18 on SNARE-mediated fusion of liposomes was studied may shed some light on this question. Here, fusion was moderately enhanced by Munc18. Enhancement was only observed with the cognate R-SNARE synaptobrevin and not with the non-cognate R-SNARE VAMP8 (Shen, Tareste et al. 2007), (Rathore, Bend et al.), although syntaxin and SNAP25 readily form complexes with VAMP8 both in vitro and in native membranes. However, considering that the effect of Munc18 requires long pre-incubation times with already preformed syntaxin/SNAP-25 complexes and is mainly observable only in the first phase of the reaction, it is unclear whether the acceleration is caused by a specific effect on SNAREs or by non-specific membrane binding as recently suggested ( $\mathrm{Xu}$, Seven et al.). Notably, it has been shown only recently that when Munc18 is added to liposomes containing only syntaxin, binding of SNAP-25 is inhibited and also liposome fusion is slowed drastically (Malsam, Seiler et al. 2009), showing that the long-known inhibitory action of Munc18-1 on the assembly of soluble SNAREs can be reproduced when SNAREs are membrane-anchored.

In another study it was reported that Munc18-1 dissociates from endogenous SNARE complexes in native membranes when exogenous synaptobrevin is added (Zilly, Sorensen et al. 2006). Thus it is conceivable that Munc18 participates in selecting the correct R-SNARE helix but dissociates upon zippering. Notably, for the SM protein Sly1, biochemical data also suggest that it might be involved in selecting the correct SNARE partners of its cognate syntaxin (Peng 2004). Hence, SM proteins might be transiently interacting with a transSNARE complex, a scenario that could unite both models of SM protein action discussed above. 


\subsubsection{SM proteins and CATCHR}

Unc13, like Unc18, was first found in the classical screen for movement mutants of $C$. elegans. Genetic ablation of Unc13 or its vertebrate homologues Munc13-1 and Munc13-2 show a lack of spontaneous and evoked release. Detailed physiological and morphological studies have shown that Munc13 is involved in docking and priming of synaptic vesicles, suggesting that Munc13 acts as a key switch that acts on the release machinery. Munc13s are large proteins that share a conserved $\mathrm{C}$-terminal region with a $\mathrm{C} 1$ domain and two $\mathrm{C} 2$ domains that flank a larger, so-called MUN domain. This domain is sufficient to rescue the total arrest in neurotransmitter release observed in hippocampal neurons lacking Munc13s. In animals, the MUN domain can be found in BAP3 and in CAPS (Unc31), which probably have a related role in vesicle priming.

The MUN domain can also be found in related proteins in many eukaryotes, suggesting that Munc13-like proteins can be traced back to the last common ancestor of all eukaryotes, in which they very probably have been involved already in docking and priming of secretory vesicles. MUN domain proteins belong to the CATCHR family members that work in various trafficking steps. Next to MUN domain proteins this family consists of the Dsl1, COG, GARP, and exocyst complexes. They form elongated arrays of $\alpha$-helical bundles with flexible hinge regions, which probably enable tethering of the transport vesicle to the site of fusion (Yu and Hughson) (Bonifacino and Glick). That Munc13 indeed belongs to the CATCHR family was demonstrated by the striking structural similarity of the crystallized portion of the MUN domain with the exocyst complex subunit Sec6p (Li, Ma et al.). Munc13, like the other tethering proteins and protein complexes, is believed to directly interact with the SNARE machinery, although so far only weak interactions, that interestingly seem to be strengthened by the presence of a membrane, have been reported. Until now, the strongest evidence for a direct role of Munc13 in the assembly of the core fusion machinery came from genetic experiments in C. elegans that showed that the LE 
mutant of syntaxin can partially rescue the requirement of Unc13 (Richmond, Weimer et al. 2001) (Hammarlund, Palfreyman et al. 2007).

Recently, it was shown that the isolated MUN domain, when added in high concentrations $(20-30 \mu \mathrm{M})$, accelerates the transition of syntaxin from the Munc18/syntaxin complex to the SNARE complex (Ma, Li et al.). This occurs, according to the authors, via a direct interaction of the MUN domain with the SNARE motif of syntaxin, through which syntaxin is rendered open. Notably, no evidence for a continuing interaction of Munc18 to the assembling SNAREs was uncovered in this study. Interestingly, the acceleration of the transition induced by the MUN domain was not sufficient to reach the speed of the transition of the LE mutant in the absence of the MUN domain ( $\mathrm{Ma}$, Li et al.), but see also (Burkhardt, Hattendorf et al. 2008). This reveals that binding of the MUN domain alone cannot account for relieving of the block exerted by Munc18 on syntaxin.

In fact, numerous studies indicate that other factors might be involved. For example, the LE mutant also partially rescues the requirement for Rim/Unc10 in C. elegans (Koushika, Richmond et al. 2001). Rim is an active zone scaffolding protein that is important for vesicle docking. It can form a tripartite complex with the N-terminal C2A domain of Munc13 and the small GTP binding protein Rab3a. It is thought that the interaction of Rim with the C2A domain of Munc13 directly activates the priming activity of Munc13. Moreover, It should be noted that the rescue by the LE mutant does not restore the mobility to the paralyzed unc13 worms. Interestingly, crossing tomosyn deletion mutants, which are characterized by having more docked vesicles, with unc13 mutants had a stronger effect on the recovery of mobility. Crossing those double deletion mutants with worms expressing the LE mutant further improved the mobility.

As mentioned above, the proteins that contain the MUN domain are members of the CATCHR family of proteins as are Dsl1, COG, GARP and the exocyst complex and help tether the vesicle to the membrane. The exocyst is a multiprotein complex found at the site of polarized secretion and regions of active cell surface expansion in mammalian cells and yeast, where it helps tether 
post-golgi vesicle destined for secretion. In mammalian cells the exocyst complex, also known as the Sec6/8 complex, localizes to areas of exocystosis in neurons and in yeast this complex is composed of eight proteins: Sec3p, Sec5p, Sec6p, Sec8p, Sec10p, Sec15p, Exo70p and Exo84p (Grote, Carr et al. 2000; He and Guo 2009).

Studies on the yeast complex have shown its importance in correct localization of vesicles to the sites of secretion by establishing a connection between the vesicle and the membrane, indeed an interaction between the vesicle bound Rab protein Sec4p and Sec15p have been reported (Salminen and Novick 1989; Guo, Roth et al. 1999). This initial interaction bridges the plasma membrane with the fusion machinery as Sec4p in turn binds to a $17 \mathrm{KDa}$ hydrophilic protein by the name of Mso1p that in turn binds to the SM family member Sec1p, which has been shown to bind to the assembled SNARE complex. This network of interactions connects directly the tethering machinery with the membrane fusion machinery, and Sec4p might well control this interaction as Sec15p binds to the active GTP bound form of Sec4p.

A Mammalian exocyst complex has also been found, the Sec6/8 complex localizes to areas of exocytosis in neurons and in epithelial cells near the tight junction (Hazuka, Foletti et al. 1999; Hsu, Hazuka et al. 1999; Shin, Zhao et al. 2000; Yeaman, Grindstaff et al. 2001) 


\subsection{Aim of the work}

Sec1/ Munc18 (SM) proteins are indispensible regulators of the docking and fusion reaction of transport vesicles. The different family members act in different trafficking steps within the cell. Probably, their main task is to control the conformational and assembly states of their respective SNARE proteins that drive membrane fusion by zippering into a tight four-helix bundle complex between the two membranes (Fig. 2) in different vesicle trafficking steps (Fig. 6). It is thought that the key binding partners of SM proteins are syntaxins (also referred to as Qa-SNAREs) that can switch between an open and closed conformation. Despite the high structural homology among SM proteins, different modes have been proposed for their association with Syntaxins (Carr and Rizo ; Halachmi and Lev 1996; Rizo and Sudhof 2002; Toonen and Verhage 2003; Verhage and Toonen 2007; Sudhof and Rothman 2009). The secretory SM protein Munc18-1, which is essential for the release of neurotransmitters from synaptic vesicles in vertebrates, binds to a "closed conformation" formed by syntaxin 1a, rendering it inaccessible to its partner SNAREs. By contrast, several SM proteins appeared at first to interact only with the N-peptide motifs of their cognate Syntaxins (Grabowski and Gallwitz 1997) (Dulubova, Yamaguchi et al. 2002; Yamaguchi, Dulubova et al. 2002; Latham, Lopez et al. 2006) (Hu, Latham et al. 2007). Recent findings, however, suggest that SM proteins might exert their regulatory role by acting on both the $\mathrm{N}$-peptide motif and the closed conformation. Whether the two binding sites act together remains unclear so far. This mode of interaction has been uncovered for the Munc18 homolog of the choanoflagellate Monosiga brevicollis, but for SM proteins involved in intracellular trafficking steps, suggesting that it comprises a general property of the interaction of SM proteins and syntaxins (Burkhardt, Hattendorf et al. 2008; Burkhardt, Stegmann et al.). 
SM proteins involved in secretion have been studied functionally in different model organisms, among them in mouse, in the yeast Saccharomyces cerevisiae and the nematode C. elegans. The mouse SM proteins Munc18-1 has also been studied biochemically and structurally in great detail. First biochemical analyses of the yeast and worm homologs showed some interesting deviations from the results obtained on Munc18-1, however. Whereas Munc18-1 binds tightly to the closed conformation of syntaxin $1 \mathrm{a}$, the contribution of the $\mathrm{N}$ peptide region that binds to outer surface of Munc18-1 is relatively small (Burkhardt, Hattendorf et al. 2008). First biochemical investigations on Unc18, the Munc18 homolog of C. elegans, demonstrated that the N-peptide region seems to be much more important for the overall affinity of the complex between Unc18 and its syntaxin partner Unc64 (Johnson, Ferdek et al. 2009). In contrast to most other SM proteins, the yeast secretory SM protein Sec1p has been reported not to be able to bind to its cognate syntaxin (Sso1) alone, but only to the already assembled SNARE complex containing Sso1p, Sec9p (SNAP-25), and Snc1/2p (Synaptobrevin) (Carr, Grote et al. 1999; Togneri, Cheng et al. 2006; Hashizume, Cheng et al. 2009).

The basis for both claims, however, is relatively weak as mainly semiquantitative protein-binding assays were used in the earlier studies. The aim of the present study was therefore to investigate the interaction of those apparently deviating secretory SM proteins in more detail by quantitative biophysical approaches like Isothermal Titration Calorimetry (ITC) and to obtain, if possible, detailed structural information on the involved proteins.

The previous studies were hampered by the fact that some of the proteins could not be expressed and purified in larger quantities, in particular the yeast protein Sec1p could not be well expressed recombinantly. In order to dissect the interactions of the secretory machinery of the nematode and baker's yeast, it was necessary to first develop strategies for obtaining the proteins in larger quantities. 


\section{Materials \& Methods}

\subsection{Chemicals, expression vectors and $E$. coli strains}

The restriction enzymes, Phusion high-fidelity DNA polymerase and T4 ligase were purchased from New England Biolabs. E. coli XL-Blue strains from Stratagene were used for cloning and storing and Bl21(DE3), BL21 (DE3)pLyss, BL21(DE3) Codon Plus were used for the expression of protein. The plasmids used in the studies are; pGEX4t (GE-Healthcare), pET28a(+) (Novagen) or pHISParallel (Addgene).

\subsection{Constructs}

\begin{tabular}{|c|c|c|c|c|}
\hline Protein & Amino acids & Vector & Resistance & Purification methods \\
\hline Sec1p & $1-723$ & pET-Duet & Ampicillin & $\mathrm{Ni}^{2+B e a d s} /$ MonoS \\
\hline Sec_Domain1 & $1-6$ & pET-28 (+) & Kanamycin & $\mathrm{Ni}^{2+}$ Beads \\
\hline$\Delta \operatorname{Sec} 1 \mathrm{p}$ & $\begin{array}{l}1-650 \\
(95-211 \text { deleted })\end{array}$ & pET-28 (+) & Kanamycin & $\mathrm{Ni}^{2+}$ Beads \\
\hline Sso1p & $1-264$ & pGEX & Ampicillin & GA-Beads/Superdex75 \\
\hline Sso1p-H3 & $180-264$ & pET-28 (+) & Kanamycin & $\mathrm{Ni}^{2+}$ Beads/MonoQ \\
\hline Mso1p & $1-210$ & pGEX-4t & Ampicillin & GA-Beads/Superdex75 \\
\hline Sec9p & $404-651$ & pET-28 (+) & Ampicillin & $\mathrm{Ni}^{2+}$ Beads/MonoQ \\
\hline Unc18 & $1-591$ & pHIS-prallel 2 & Ampicillin & $\mathrm{Ni}^{2+}$ Beads/Superdex75 \\
\hline Unc64_LE & $1-263$ & pHIS-prallel 2 & Ampicillin & $\mathrm{Ni}^{2+B e a d s / S u p e r d e x 75}$ \\
\hline Unc64_L9A & $1-263$ & pHIS-prallel 2 & Ampicillin & $\mathrm{Ni}^{2+B e a d s / S u p e r d e x 75}$ \\
\hline Unc64_1-180 & $1-180$ & pHIS-prallel 2 & Ampicillin & $\mathrm{Ni}^{2+B e a d s / S u p e r d e x 75}$ \\
\hline Unc64_1-227 & $1-227$ & pHIS-prallel 2 & Ampicillin & $\mathrm{Ni}^{2+B e a d s / S u p e r d e x 75}$ \\
\hline Unc64_I234A & $1-263$ & pHIS-prallel 2 & Ampicillin & $\mathrm{Ni}^{2+B e a d s / S u p e r d e x 75}$ \\
\hline Unc64 & $1-263$ & pGEX & Ampicillin & GA-Beads/Superdex75 \\
\hline
\end{tabular}

Table1. Summary of protein constructs used in the studies, expression vectors and purification methods 


\subsection{Protein expression and purification}

In order to obtain the proteins to be used by recombinant means it was necessary to first transform the E. coli strains with the corresponding plasmid or plasmids as is the case for co-transformation. $10 \mathrm{ng}$ of DNA total (5ng $+5 \mathrm{ng}$ for co-transformation) added to the cells was incubated on ice with thawed E. coli cells of the selected chemically competent strains and incubate for 15 mins, after the incubation the cells were transferred to a water bath set to $42^{\circ} \mathrm{C}$ for 45 secs in order to expose the cells to a heat shock. The cells were incubated further in $0.5 \mathrm{ml}$ Luria Bertani (LB) medium (1\% (w/v) tryptone, 0.5\% (w/v) yeast extract, $1 \%\left(\mathrm{w} / \mathrm{v}\right.$ ) $\mathrm{NaCl}, \mathrm{pH} ; 7.0$ ) (Formedium, United Kingdom) at $37^{\circ} \mathrm{C}$ for one hour before plated on LB agar plates and let grow at $37^{\circ} \mathrm{C}$ over night.

In order to over-express the desired gene in vitro a single colony was selected and inoculated in LB medium and grown over night at $37^{\circ} \mathrm{C}$ under constant shaking at $180 \mathrm{rpm}$, a selected antibiotic was added to the media depending on the resistance gene present in the plasmid. Kanamycyn at 30 $\mu \mathrm{g} / \mathrm{ml}$ and-or Ampicillin at $100 \mu \mathrm{g} / \mathrm{ml}$. From the overnight pre-culture $5 \mathrm{ml}$ were added to $500 \mathrm{ml}$ (1:100) LB media or Terrific Broth (TB) medium (1.2\% (w/v) tryptone, $2.4 \%(\mathrm{w} / \mathrm{v})$ yeast extract, $0.4 \%(\mathrm{w} / \mathrm{v})$ glycerin and $10 \%(\mathrm{v} / \mathrm{v})$ (Formedium, United Kingdom) Phosphate buffer composed of $0.17 \mathrm{M} \mathrm{KH}_{2} \mathrm{PO}_{4}$ and $0.72 \mathrm{M} \mathrm{K}_{2} \mathrm{HPO}_{4}$ ) containing the corresponding antibiotic and let to grow at $37^{\circ} \mathrm{C}$ under constant shake at $180 \mathrm{rpm}$ until an $\mathrm{OD}_{600}$ of 0.4-0.8 for $\mathrm{LB}$ and 0.8-1.0 for TB was reached prior to the addition of $1 \mathrm{mM}$ IPTG (Fluka, Switzerland) for induction and let over night at the temperature chosen as optimal for the particular protein. After induction, the cells were harvested by centrifugation at $3500 \mathrm{rpm}$ for 20 mins and later re-suspended in the buffer that will be used on the first purification step, this being either $\mathrm{Ni}^{2+}$ washing buffer $(20 \mathrm{mM}$ Tris- $\mathrm{HCl}$ pH 8.0, 500mM NaCl, 30mM Imidazole) or Glutathione-buffer (20mM Tris- $\mathrm{HCl}$ pH 8.0, 500mMNaCl, 1mM DTT) and the cells were either lyzed of stored at $20^{\circ} \mathrm{C}$. 
For isolation and purification of the expressed protein the pellet was thawed or resuspended and an additional PMSF and $1 \mathrm{mg} / \mathrm{ml}$ lysozyme was added to the suspension together with $5 \mathrm{mg}$ DNaseI, $1 \mathrm{mM} \mathrm{MgCl}_{2}$ and $1 \%(\mathrm{v} / \mathrm{v})$ Triton X-100 before lysis by sonification (Branson Sonifier), in addition 6M Urea was added to SNARE cell suspension. Cellular debris was later cleared from the lysate by centrifugation at $13000 \mathrm{rpm}$ for 1 hour and the soluble protein in the supernatant was incubated with either $\mathrm{Ni}^{2+}$-NTA or Glutathione sepharose beads for 2 hours at $4^{\circ} \mathrm{C}$ under rotation. The incubated $\mathrm{Ni}^{2+}$ beads are collected and washed with $100 \mathrm{ml} \mathrm{Ni}^{2+}$ washing buffer and eluted with $10 \mathrm{ml}$ elution buffer (20mM Tris- $\mathrm{HCl} \mathrm{pH} 8.0,500 \mathrm{mM} \mathrm{NaCl}$ and 400mM Imidazole).

The Unc-64construct contains a GST tag and is therefore incubated with Glutathione sepharose (GE Healthcare) beads were used and washed instead with $100 \mathrm{ml}$ Glutathione- buffer and cleaved off the beads by incubating the washed beads with TEV (Tobacco Etch Virus) protease overnight at $4^{\circ} \mathrm{C}$ under constant rotation. The cleaved protein is collected afterwards and purified further by means of size exclusion chromatography using a Superdex-75 equilibrated in $20 \mathrm{mM}$ Tris- $\mathrm{HCl}$ pH8.0, $500 \mathrm{mM} \mathrm{NaCl}$ and $1 \mathrm{mM}$ DTT. The eluted fractions containing Unc64 were pooled and stored at $-80^{\circ} \mathrm{C}$

For the other proteins that require ion exchange chromatography the method (MonoQ HR or MonoS HR by GE-healthcare) was chosen according to the $\mathrm{pI}$ of the protein, the protein was dialysed against Buffer A $(20 \mathrm{mM}$ Tris- $\mathrm{HCl} \mathrm{pH}$ 8.0, 1 $\mathrm{mM} \mathrm{DTT,} 100 \mathrm{mM} \mathrm{NaCl}$ ) and loaded onto the column with an AKTA explorer purification system. The protein was washed with 2 Column Volumes of Buffer A before eluted with a linear gradient produced with Buffer A and Buffer B (20mM Tris-Hcl pH 8.0, 1mM DTT, $1 \mathrm{M} \mathrm{NaCl}$ ). The purification methods used for every individual protein are presented in table1. 


\subsection{Determination of protein concentration}

The protein concentration was estimated by UV-Spectroscopy measuring the absorption at a wavelength range between 220-350nm with a Nanodrop (Thermo Scientific) from which the absorption value at the wavelength of $280 \mathrm{~nm}$ was recorded and used to calculate the protein concentration, the theoretical extinction coefficient used was calculated using the ProtParam tool software from the SwissProt database (www.swissprotparamtool.com; Wilkins et al., 1999),

\subsection{GST Pull Downs}

The components used in these studies were expressed by recombinant means and mixed in a 1:1:1:1 ratio and incubated for 2 hours at $4^{\circ} \mathrm{C}$ on HeadOver-Tail rotation and let assemble into a complex in the presence of beads with an Sso1p-GST bound construct. The proteins were pulled out with the Sso1p-GST construct by means of centrifugation (2 $000 \mathrm{rpm}$ at $4^{\circ} \mathrm{C}$ for $5 \mathrm{mins}$ ) and washed 3 times with a buffer composed of 20mM Tris- $\mathrm{HCl}, 100 \mathrm{mM} \mathrm{NaCl}, 1 \mathrm{mM}$ DTT, pH 8.0, before eluted with wash buffer containing an additional $10 \mathrm{mM}$ Glutathione and later analyzed by SDS-PAGE.

\subsection{SNARE complex formation analysis}

The components used in these studies were expressed by recombinant means and mixed in a 1:1:1:1 ration in a buffer composed of $20 \mathrm{mM}$ Tris- $\mathrm{HCl}$, $100 \mathrm{mM} \mathrm{NaCl}, 1 \mathrm{mM}$ DTT, pH8.0 and incubated for 2 hours at $4^{\circ} \mathrm{C}$ on Head-OverTail rotation in a similar manner as the Pull-down studies, the binding reactions were analyzed by Native gel electrophoresis as the yeast SNARE complex is not SDS resistant. 


\subsection{Membrane binding assay}

\subsubsection{Liposome preparation}

The 2x lipid solution containing 20\% PS: 20\% PE: 10\% Cholesterol: 50\% PC was dissolved in 20mM HEPES pH 8.0, 150mM KCl, 5\% Na-Cholate and 5mM DTT with a $13.5 \mathrm{mM}$ total lipid concentration. The $2 \mathrm{x}$ lipid solution was mixed with either buffer solution in a $1: 1(\mathrm{v} / \mathrm{v})$ to obtain a $1 \mathrm{x}$ final lipid solution or a protein solution to create a $1 \mathrm{x}$ final lipid solution and a 1:300 lipid to protein ratio. After the protein is added to the lipid solution the mixture is incubated for 10 at room temperature and later spun at $13000 \mathrm{rpm}$ for $10 \mathrm{mins}$ before injecting into a Fast Desalting PC 3.2/10 column at a flow rate of $500 \mu \mathrm{l} / \mathrm{min}$.

\subsubsection{Floatation assay}

In order to assess whether the presence of a membrane provides the interaction with the right environment to promote a binding, floatation assays were set to study the localization of the proteins on a Nycodenz gradient. The fraction in which the protein is found reflects the level of binding towards the membrane or a component that is anchored to the membrane. Both the Nycodenz solutions (80\% and 30\%) and Protein solution are dissolved in HEPES-buffer (20mM HEPES pH 8.0, 1mM TCEP, 150mM NaCl) and the components are mixed as follow:

$25 \mu$ l Protein solution

$25 \mu \mathrm{l}$ Liposome solution

$50 \mu \mathrm{l} 80 \%$ Nycodenz Mix

$50 \mu \mathrm{l} 30 \%$ Nycodenz

$30 \mu$ l Buffer solution 
The mixture whit the carefully overlaid 30\% Nycodenz and $10 \mu \mathrm{l}$ Buffer solution were spun at $45000 \mathrm{rpm}$ for 90 minutes before the top layer and the bottom layer were carefully removed and analyzed by SDS PAGE electrophoresis.

\subsection{Isothermal Titration Calorimetry (ITC)}

All the measurements were carried out in in a VP-ITC (MicroCal) at $25^{\circ} \mathrm{C}$ according to the manufacturers instructions and in phosphate buffer $(20 \mathrm{mM}$ sodium phosphate buffer at pH7.4, $150 \mathrm{mM} \mathrm{NaCl}$ ) in which all the protein were dialyzed extensively, the proteins and the buffer to be used in the studies were both filtered and degassed prior to use. The data was analyzed with the Origin derived software provided by the company (MicroCal Origin 7.0) and fitted with a non-linear least square fit for a single site-binding model.

The instrument is composed of two cells enclosed and isolated in an adiabatic shield jacket; the sample cell and the reference cell. The reference is usually filled with buffer or water, while the other is filled with one of the binding partners, through a feedback mechanism composed of a thermophile or thermocouple system, the sample cell is constantly being compared to the buffer filled reference cell. As the protein in the syringe (the ligand) is injected into the sample cell, where its diluted binding partner is (the receptor), heat will be produced or taken away depending on the nature of the binding and a difference between the sample cell vessel and the reference cell will arise, the machine will detect and monitor this difference an try to compensate for this loss or gain by applying power into the sample cell or reducing the input of power in order to regain the balance between the two cells.

An ITC machine operates according to the dynamic power compensation principle, meaning that it tries to maintain the temperature difference between both the sample cell and the reference cell constant and close to zero by compensating for any heat that is taken away by adding it if the reaction is endothermic, or to stop the power input when heat is taken away during the 
binding interaction if it is an exothermic reaction and use of the Peltier effect for cooling. This event will be detected and reported by the instrument through a feed-back mechanism and recorded by a computer and the changes in heat will be reflected on as a peaks, with the initial deviation from the baseline produced by the heat change cause by the interaction, and the second by the machine as it tries to restore the thermal equilibrium and bring the signal back to baseline. These peaks will be continued to be produced with the step-wise injection of the ligand into the sample cell and will decrease in size as a smaller change in heat is produce due to a decrease of free binding sites as the reaction reaches saturation, the peaks will be later integrated with respect of time and the result from this integration will produce a point in a plot against the molar ratio of the components giving rise to an isotherm with a curve that is then fitted to the binding model that describes the interaction. 


\section{$2.9 \quad$ Gel electrophoresis}

\begin{tabular}{|l|l|l|l|l|}
\hline Component & \multicolumn{3}{|c|}{ Running gel } & Stacking gel \\
\hline Percentage & $\mathbf{8 \%}$ & $\mathbf{9 \%}$ & $\mathbf{1 5 \%}$ & $\mathbf{3 . 7 5 \%}$ \\
\hline Running gel buffer (pH 8.8) & $2.5 \mathrm{ml}$ & $2.5 \mathrm{ml}$ & $2.5 \mathrm{ml}$ & \\
\hline Stacking gel buffer (pH 6.8) & & & & $2.5 \mathrm{ml}$ \\
\hline H20 & $4.7 \mathrm{ml}$ & $4.4 \mathrm{ml}$ & $2.4 \mathrm{ml}$ & $6.14 \mathrm{ml}$ \\
\hline Acrylamide/bis-acrylamide & $2.7 \mathrm{ml}$ & $3.0 \mathrm{ml}$ & $5.0 \mathrm{ml}$ & $1.25 \mathrm{ml}$ \\
\hline TEMED & $5 \mathrm{ul}$ & $5 \mathrm{ul}$ & $5 \mathrm{ul}$ & $10 \mathrm{ul}$ \\
\hline $10 \%$ APS & $100 \mathrm{ul}$ & $100 \mathrm{ul}$ & $100 \mathrm{ul}$ & $100 \mathrm{ul}$ \\
\hline
\end{tabular}

Table2. SDS-PAGE recipe

Recipe used for preparing the SDS-PAGE and native gels in which the stacking gel buffer contains $0.5 \mathrm{M}$ Tris pH 6.8 with $0.4 \%$ SDS while the running gel contains $1.5 \mathrm{M}$ Tris pH 8.8 with $0.4 \%$ SDS. The same recipe is used for making native gel with the difference that neither the running gel buffer nor the stacking gel buffer contains SDS.

SDS-PAGE (15\%) and native gels (15\%) were prepared according to the recipe in table 2. The gel was later fixed and stained with $(50 \%(\mathrm{v} / \mathrm{v})$ methanol, $10 \%(\mathrm{v} / \mathrm{v})$ acidic acid and 0.2\% (w/v) Coomassie Brilliant Blue R-250). The gel was first destained in De-stain Solution 1 (50\% (v/v) ethanol and 10\% (v/v) acidic acid) and later with De-stain Solution $2(5 \%(\mathrm{v} / \mathrm{v})$ methanol and 12.5\% (v/v) acetic acid). 


\section{Results}

\subsection{Characterization of the C. elegance Unc18 interaction to Unc64}

The members of the sec1/Munc18 family of proteins have been shown to work through the binding of their cognate Qa-SNAREs and help promote fusion. Due to the high level of sequence conservation between the couples Munc18a/Syntaxin1a from Rattus norvegicus, and Unc18/Unc64 from C. elegans (58\% and 64\% identical amino acid respectively) a mutational study was carried out based on the findings obtained for the Munc18/Syntaxin couple in order to gain deeper insights into the binding mode exerted in the Unc18/Unc64 interaction. The initial studies to investigate the binding mode used by the Unc18 and Unc64 in vitro were performed by means of Isothermal Titration Calorimetry (ITC). For this approach different fragments and mutants of Unc64 were used: equivalent regions and mutants were tested and have been reported to affect the binding of Munc18 to Syntaxin (Fig. 7). (Burkhardt, Hattendorf et al. 2008) (Misura, Scheller et al. 2000) (Dulubova, Sugita et al. 1999; Johnson, Ferdek et al. 2009)). 


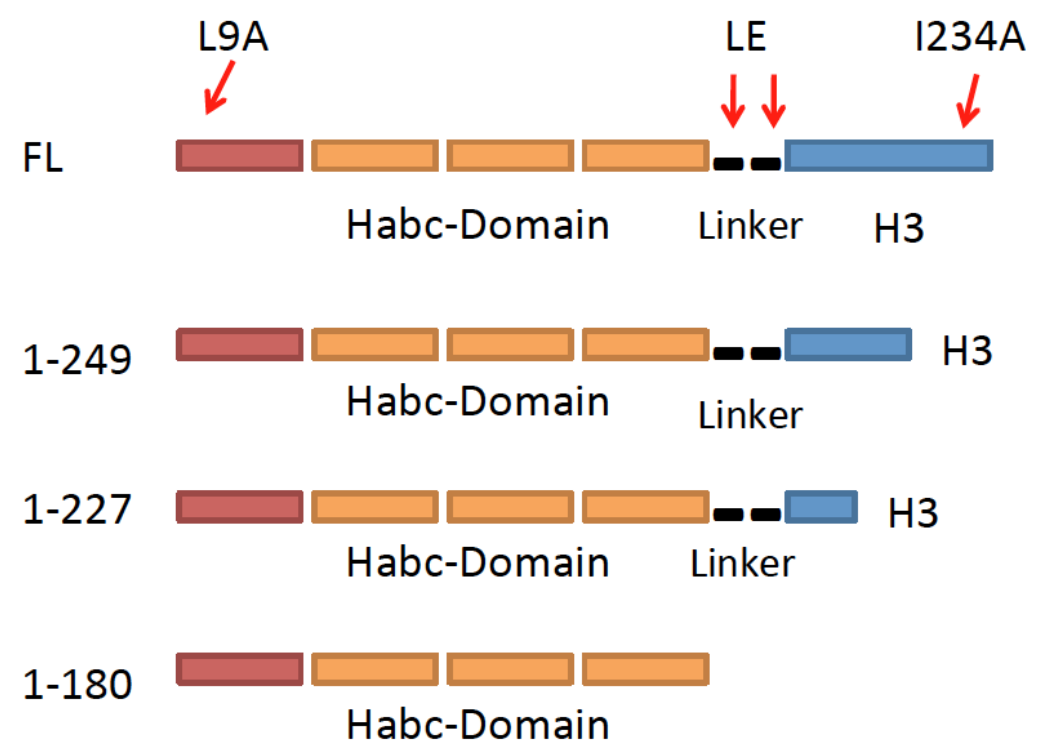

Figure 7. Schematic diagram of Unc64 constructs used in the interaction studies to Unc18.

The constructs used are based on regions that have been reported to affect binding in the mammalian pair Munc18/Syntaxin1a. The Unc64 full length protein (FL) consist in the region 1-263 which is composed of the N-peptide binding region in red, the Habc region in orange, the linker region represented by the black dash lines and the SNARE motif shown in blue. The construct lacks the trans-membrane region.

The mutations inserted individually correspond to L9A and is the equivalent to L89 in Syntaxin1a and has been shown to affect the interaction of the $\mathrm{N}$-peptide to the binding pocket, the LE double mutant correspond to the originally discovered L164A/L165A that has been shown to create constantly active Syntaxin that bypasses the inhibitory effect of Munc18. The last point mutation corresponds to a residue in the SNARE motif and that has been shown to create the same effect as the truncation of the entire SNARE motif. The truncated version of Syntaxin (1-249), (1-227) and (1-180) were designed to investigate the importance of the SNARE motif on the interaction as it has been reported to bind only through the Npeptide and therefore without the need of the SNARE motif.

\subsubsection{Unc18 binds to Unc64 with high affinity and requires the $\mathrm{N}$-peptide}

As has been shown in the mammalian system (Burkhardt, Hattendorf et al. 2008) and in accordance with in vivo studies in C. elegance (Johnson, Ferdek et al. 2009), Unc18 binds to its cognate Syntaxin Unc64 in a 1:1 fashion. In addition a Kd of $31 \mathrm{nM}$ and a change in energy of $-55 \mathrm{kcal} /$ mole was observed (Fig. $8 \mathrm{~A}$ and table 3). 
A

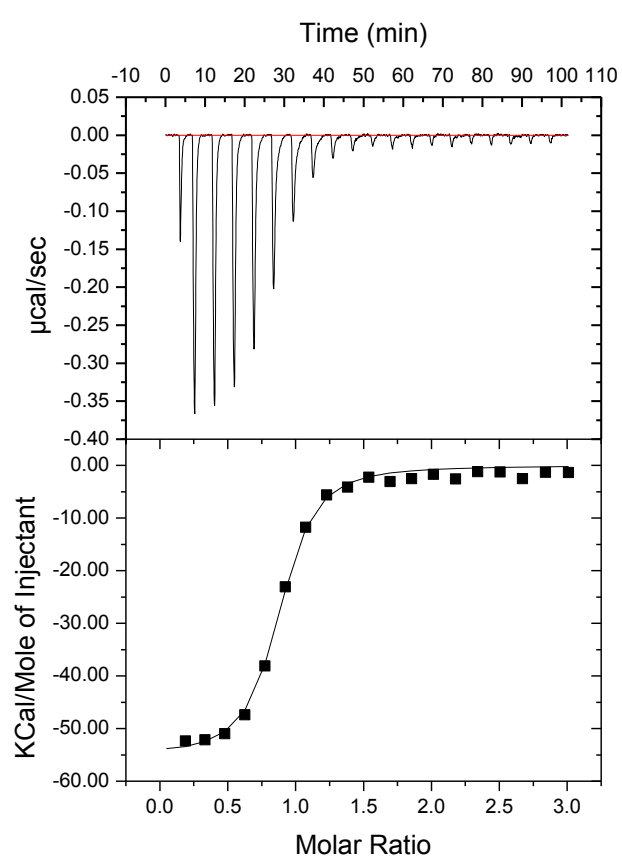

B

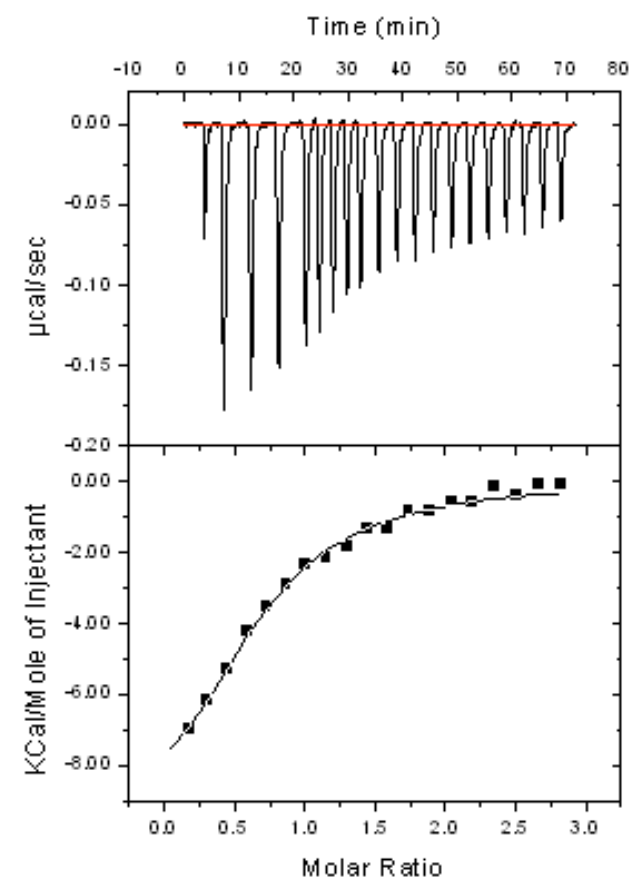

Figure 8. Calorimetric titration of Unc64 and Unc64 (L9A) with wild type Unc18

The diagrams show the ITC experiments in which the cytosolic region of Unc64 (A) and the same region with the point mutation L9A (B) were titrated into Unc18. In figure (A) the wild type Unc64 was titrated into Munc18 until saturation was reached with the upper panel showing the corresponding heat change of every injection and the lower panel the integrated area of every peak that is represented by a point in the curve. In figure (B) the same method was applied but here it is clear that the replacement of the Lysine residue into Alanine causes a defect in the interaction, lowering the affinity and heat released per injection.

In addition, when a mutation that interferes with the binding of the $\mathrm{N}$ peptide region in the Munc18a/Syntaxin1a couple (L8A) is introduced into Unc64 (L9A), a comparable loss in binding capability was observed. Although Unc64 (L9A) was still able to interact with Unc18, the high affinity observed for the wild type (Fig. 8A) is lost (Fig. 8B), highlighting the importance of an intact $\mathrm{N}$-peptide for tight binding. This finding is in accordance with previous reports in which it was proposed that Unc18 binds to Unc64 in a manner that requires the N-peptide (Johnson, Ferdek et al. 2009). 


\subsubsection{Truncation of the SNARE motif severely impairs the binding of Unc64 to Unc18}

Binding of mammalian Munc18a to syntaxin 1a requires an intact SNARE motif in syntaxin (Burkhardt, Hattendorf et al. 2008) (Misura, Scheller et al. 2000), with which it forms a closed conformation with the Habc domain. To investigate whether binding of Unc18 also needs an intact SNARE motif of Unc64 for proper interaction, a series of truncations were made in the SNARE motif Cterminal region (Fig. 9A-C).

Already the removal of the last 14 residues of the $\mathrm{C}$-terminal region of the Unc64 SNARE motif (from 1-263 to 1-249) led to a reduction in affinity from $31 \mathrm{nM}$ to $9 \mu \mathrm{M}$ and a decrease in energy released from $-55 \mathrm{kcal} / \mathrm{mole}$ to $-32 \mathrm{kcal} /$ mole. As the region of the SNARE motif was shortened even further down to residue 227, the interactions was completely abolished. An abolition of the complex formation was not observed in the mammalian system when it was truncated to the equivalent residue 226, but was only seen to be severely impaired by a drop in affinity by a 100 -fold. For the mammalian proteins an interaction was still detected even as the protein was shortened down to residue number 170 in Syntaxin (Burkhardt, Hattendorf et al. 2008). As expected, further truncation of Unc64 down to 180 showed no interaction with Unc18. 
A

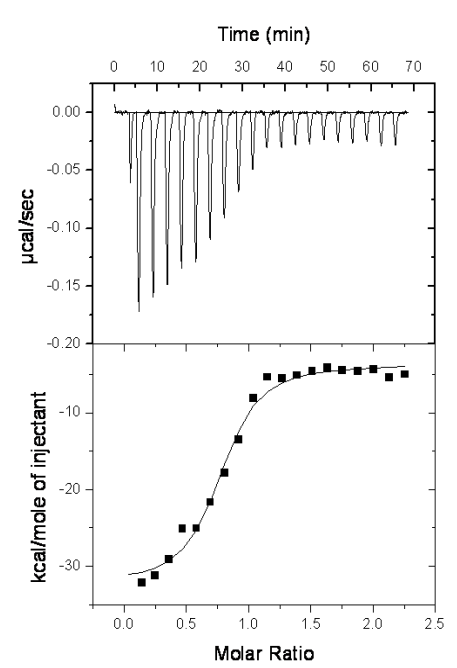

B

227

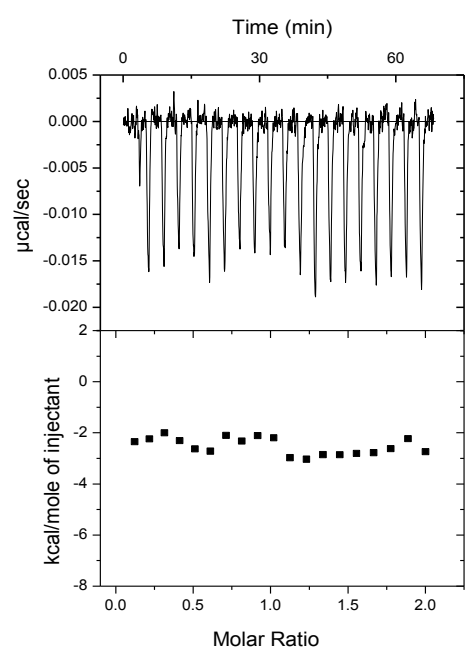

C

180

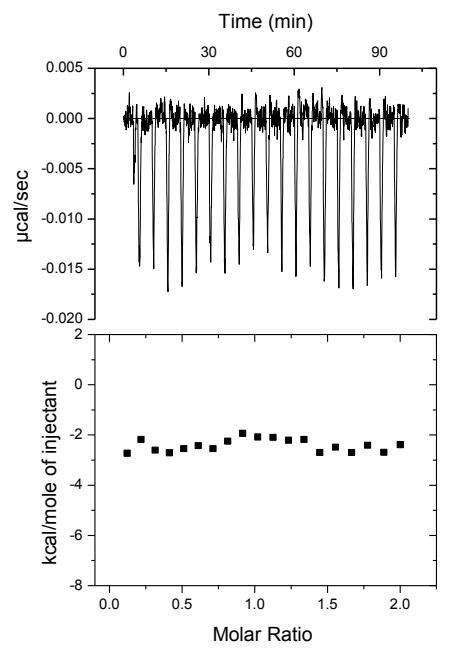

Figure 9. Calorimetric titration of truncated versions of Unc64 with wild type Unc18

The results from the ITC experiments with truncated versions of Unc64 show that the interaction is only slightly affected when the SNARE motif is shortened until residue 249 (A), while it is severely affected when it is truncated until residue 227 (B) and 180 (C). This is in accordance with a binding mode of Unc18 that requires the four-helix bundle and therefore resemble the mode of action previously shown for the Munc18a/Syntaxin1a, that require both the N-peptide and the four-helix bundle (Burkhardt, Hattendorf et al. 2008).

It has previously been reported that a key Isoleucine found embedded in the SNARE motif of Syntaxin (I233) plays a crucial role in the interaction between Syntaxin1a and Munc18a (Burkhardt, Hattendorf et al. 2008) (Misura, Scheller et al. 2000), in ordered to study the effect that this residue has in the $C$. elegance system a replacement to an Alanine was done in the equivalent I234 in Unc64 (1234A). Although the interaction is preserved in the mammalian system with an affinity that is substantially affected, the interaction between Unc18 and Unc64 was abolished all together (Fig. 10A), showing yet again a strong dependence of the interaction on an intact SNARE domain which is in accordance with a binding mode that require the four-helix bundle.

In addition to these studies a so-called "open mutant" L165A/E166A that creates a Syntaxin that bypasses the regulatory effect of Munc18 and is therefore 
constantly active both in vivo and in vitro (Burkhardt, Hattendorf et al. 2008) (Johnson, Ferdek et al. 2009) (Dulubova, Sugita et al. 1999; Gerber, Rah et al. 2008), was created in Unc64 (L166A/L167A). The LE mutant in C. elegans shows a slight decrease in binding affinity and heat released (from -55 to -30 $\mathrm{kcal} / \mathrm{mole}$ ) (Fig. 10B), comparable to the results obtained for the mammalian homolog.

A

$\mathrm{I} 234 \mathrm{~A}$

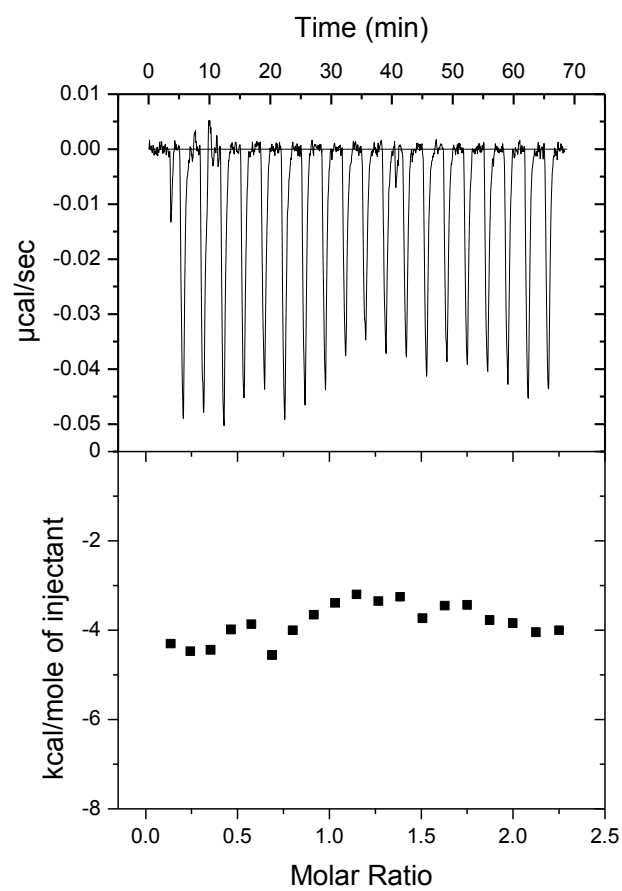

$\mathrm{B}$

LE

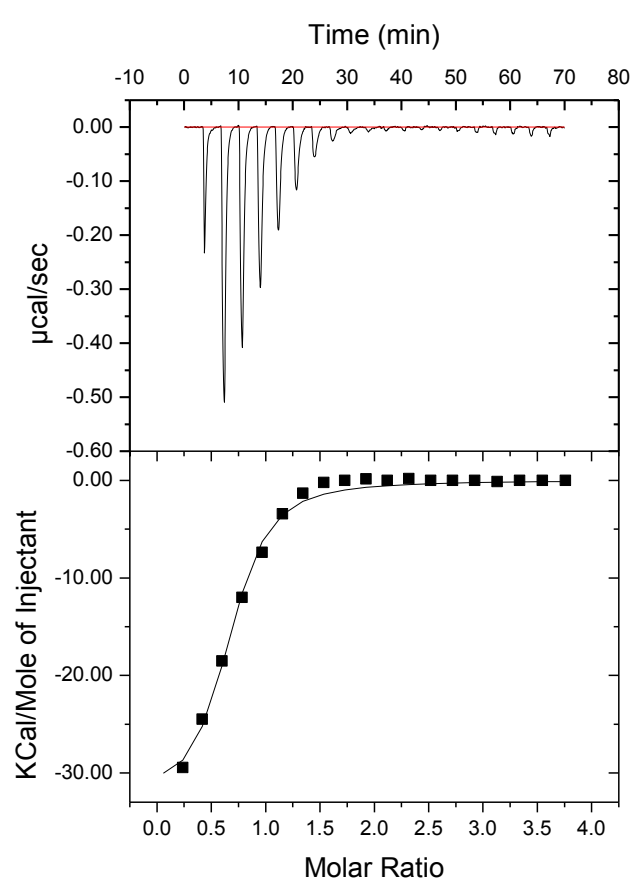

Figure 10. Calorimetric titration of Unc64 (I234A) and Unc64 (LE) with wild type Unc18

The results from the ITC experiments with the point mutation I234A that shows a complete abolition of the interaction between Unc18 and Unc64 (A) and it is in full accordance with the previous results in with truncation experiments showing a preserved interaction until truncation 1-227 (Fig 9B), the truncation experiments could be explained by either not having enough region for the Unc18 to bind or simply because the amino acid Isoleucine 234 is missing. The interaction between Unc18 and the LE double mutant shows the same amount of heat development but the interaction gets saturated within the first 10 injections which could be due intramolecular interactions between the mutants. 
In conclusion, these results show that the binding mode that Unc18 uses to bind Unc64 resembles the one used by Munc18a when binding to Syntaxin1a, indicating that there is a conserved mechanism that could be detected due to the sensitivity of our methods.

\begin{tabular}{|l|l|l|l|}
\hline syringe & Cell & Affinity $(\boldsymbol{\mu M})$ & $\boldsymbol{\Delta}$ (kcal/mole) \\
\hline Unc64 (WT) & Unc18 & $0.031 \pm 0.2$ & $55.2 \pm 0.93$ \\
\hline Unc64 (L9A) & Unc18 & $1.2 \pm 7.6$ & $11.2 \pm 1.0$ \\
\hline Unc64 (I233A) & Unc18 & - & - \\
\hline Unc64 (LE) & Unc18 & $0.169 \pm 0.94$ & $32.9 \pm 1.2$ \\
\hline Unc64 (1-249) & Unc18 & $9.7 \pm 0.0$ & $32.3 \pm 2.8$ \\
\hline Unc64 (1-227) & Unc18 & - & - \\
\hline Unc64 (1-180) & Unc18 & - & - \\
\hline
\end{tabular}

Table 3. Summary of the thermodynamic parameters obtained from the all the ITC experiments performed

\subsection{Mapping of the areas of interaction in Unc64 by Radical Foot- printing in combination with Mass-spectrometry analysis}

To further investigate which regions are involved in the complex formation between Unc18 and Unc64, radical footprinting combined with mass spectrometry was used (Takamoto and Chance 2006; Kiselar and Chance 2010; Wang and Chance 2011). This method is useful to map areas that are involved in creating a physical interactions. Radical footprinting is a method based on the propensity of residues to chemical modifications on their side chains as they are exposed to chemical radicals, the difference in modification pattern are compared between the single subunits and the complex in order to catalogue the areas that get protected upon complex formation.

Since there is no solved crystal structure of either the Unc18/Unc64 complex nor the single components, we wanted first and foremost see if the binding mode that was proposed by the ITC studies could be confirmed and at 
the same time look for other novel areas that are important for the interaction and have not been reported. Hydroxyl radicals were generated by laser photolysis of hydrogen peroxide with a single nanosecond-scale pulse. Excess radicals were depleted with D-L methionine present in the solution. Radicals are therefore short-lived and can only modify side chains in their vicinity before being scavenged. This method, termed FPOP (flash photolysis of peroxide) probes protein and protein complexes in a nanosecond to microsecond timescale, which ensures structural integrity, since these timescales are normally shorter than the needed for folding and unfolding events. The modification patterns in the Unc18/Unc64 complex and single subunits are summarized in table 4 .

\begin{tabular}{|c|c|c|c|c|c|c|c|c|c|c|}
\hline \multirow{3}{*}{$\#$} & \multirow{3}{*}{ Sequence } & \multirow{3}{*}{ Peptide } & \multicolumn{6}{|c|}{ UNC-64 } & \multirow{3}{*}{$\begin{array}{c}\text { Difference } \\
\text { significant? } \\
(\mathrm{Y} / \mathrm{N})\end{array}$} & \multirow{3}{*}{ Residues exposed } \\
\hline & & & \multicolumn{3}{|c|}{ Single Protein } & \multicolumn{3}{|c|}{ Complex } & & \\
\hline & & & A & B & c & A & B & c & & \\
\hline 1 & $11-43$ & AAQSEDEQDDDMHMDTGNAQYMEEFFEQVEEIR & ND & ND & ND & ND & ND & u & & \\
\hline 2 & $248-253$ & AVADTKK & ND & $\mathrm{u}$ & ND & u & u & $\mathrm{u}$ & & \\
\hline 3 & $255-261$ & AVQYask & $\mathrm{u}$ & u & $\mathrm{u}$ & u & u & u & & \\
\hline 4 & $4-10$ & DRLSALK & $\mathrm{M}$ & $\mathrm{M}$ & $u$ & $u$ & $u$ & $u$ & $\mathrm{~N}$ & D4 \\
\hline 5 & $243-253$ & EFVDRAVADTK & $\mathbf{u}$ & $u$ & $u$ & $u$ & $u$ & $u$ & & \\
\hline 6 & $212-242$ & ELHDMFMDMAMLVESQGEMVDRIEYNVEHAK & ND & ND & ND & $u$ & ND & ND & & \\
\hline 7 & 128-137 & FVEVMTDYNK & $\mathrm{M}$ & $\mathrm{M}$ & $\mathrm{M}$ & M & $\mathrm{M}$ & $\mathrm{M}$ & Y & M132, D134, Y135 \\
\hline 8 & $44-57$ & GSVDIIANNVEEVKK & $u$ & $u$ & $u$ & $u$ & $u$ & u & & \\
\hline 9 & $200-211$ & HNDIMKLESSIR & M & M & M & M & M & M & Y & M204 \\
\hline 10 & $60-72$ & HSAILSNPVNDQK & $\mathrm{u}$ & $\mathrm{u}$ & $\mathrm{u}$ & $\mathrm{u}$ & $\mathrm{u}$ & $\mathrm{u}$ & & \\
\hline 11 & $234-242$ & IEYNVEHAK & $u$ & $u$ & $u$ & $u$ & $u$ & $u$ & & \\
\hline 12 & $150-159$ & IQRQLDIAGK & u & $\mathrm{u}$ & $u$ & u & u & $u$ & & \\
\hline 13 & $97-115$ & LIENAIDHDEQGAGNADLR & M & $\mathrm{M}$ & $u$ & $u$ & $u$ & $u$ & N & E99, D103, H104, Q107 \\
\hline 14 & 153-159 & QLDIAGK & $u$ & $u$ & $u$ & $u$ & $u$ & $u$ & & \\
\hline 15 & 191-199 & QTLADIEAR & $\mathrm{M}$ & $\mathrm{M}$ & ND & u & u & u & N & L193 \\
\hline 16 & $160-190$ & QVGDEDLEEMIESGNPGVFTQGIITDTQQAK & $\mathrm{M}$ & $\mathrm{M}$ & $u$ & $\mathrm{M}$ & M & $\mathrm{M}$ & $\mathbf{Y}$ & M169 \\
\hline 17 & $127-137$ & RFVEVMTDYNK & $\mathrm{M}$ & $\mathrm{M}$ & $\mathrm{M}$ & $u$ & $\mathrm{M}$ & $\mathrm{M}$ & $\mathbf{Y}$ & M132 \\
\hline 18 & $73-85$ & TKEELDELMAVIK & $\mathrm{M}$ & $\mathrm{M}$ & $M$ & $\mathrm{M}$ & $\mathrm{M}$ & $\mathrm{M}$ & $Y$ & M81 \\
\hline 19 & $119-126$ & TQHSTLSR & ND & $u$ & u & u & u & u & & \\
\hline 20 & $138-145$ & TQTDYRER & u & $\mathrm{u}$ & u & u & $\mathrm{u}$ & u & & \\
\hline
\end{tabular}

\section{Table 4. Summary of residue modification on Unc64 upon complex formation}

The summarized results of peptide modification of the unbound Unc64 and the difference in modification upon complex formation. Although there are some regions that were not detected (ND) there were three that showed a significant change in the modification pattern upon complex formation. Peptide 4, corresponding to the N-peptide region of Unc64 (visualized in figure 11). Peptide 13 corresponds to an area that aligns to a loop region between helix $\mathrm{Hb}$ and $\mathrm{Hc}$ in Syntaxin and found covered by Munc18 on the structure. Suggesting that Unc64 might be covered in the same way. Peptide 15 corresponds to a region that would correspond to the beginning of the H3 domain in Syntaxin1a, this reveals a new region that can give us more information on the way Unc64 binds to Unc18 and perhaps a more general binding step.

The experiments were performed in triplicates and the residues that were modified (M) or unmodified (U) were annotated together with the degree of significance of the modification. " $Y$ " as in "yes" for a significant difference and " $\mathrm{N}$ " for no significant difference. 
Due to the high level of sequence conservation between Munc18 and its cognate SNARE Syntaxin1a, and the C. elegance Unc18 and its binding SNARE Unc64 (58\% and 64\% identical amino acid respectively) which in addition seem to require the same binding regions, the sequences of the modified peptides were inserted into the crystal structure of Munc18/Syntaxin1a by hand in order to get some orientation of the areas they correspond to. (PDB:3C98) (Burkhardt, Hattendorf et al. 2008).

Laser Probing of UNC18-UNC64 and [UNC18-UNC64]Complex

UNC18-A: Sequence Coverage: 90.9\%

>gi|435805|gb|AAB28570.1| UNC-18 [Caenorhabditis elegans]

MSLKQIVGHKLLNDVIRPLKKGDGRSAWNVLIVDTLAMRMLSSCCKMHNIMEEGITIVEDLNKRREPLPTLEAIYLIAP TAESIDKLIQDYCARNLYKCAHVFFTEACSDQLFSTLSKSAAARFIKTLKEINLAFTPYESQVFNLDSPDTFFLYYNAQKQ GGLTSNLERIAEQIATVCATLGEYPSLRYRADFERNVELGHLVEQKLDAYKADDPSMGEGADKARSQLIIIDRGYDAITP LLHELTLQAMCYDLLGIENDVYKYETGGSDENLEKEVLLDENDDLWVEMRHKHLAVVSQEVTKNLKKFSESKGNKGT MDSKSIKDLSMLIKRMPQHKKELNKFSTHISLAEECMKQYQQGVDKLCKVEQDLSTGIDAEGERVRDAMKLMVPLLID PAVRCEDRLRLILLYILSKNGITDENLNKLLQHANISMADKETITNAAYLGLNIVTDTGRKKTWTPTKKERPHEQVYQS SRWVPVIKDIEDAIDERLDTKHFPFLAGRQVNQGYRAPASARYGQWHKERGQQSNYRSGPRLIIYIGGVTFSEMRACY EVTAARKPWEVVIGSDRIITPDKFLTNLRDLNKPRDI

UNC64-A: Sequence Coverage: 72.5\%

>gi|74956578|sp|016000.1|STX1A_CAEEL RecName: Full=Syntaxin-1A homolog; AltName:

Full=Uncoordinated protein 64

MTKDRLSALKAAQSEDEQDDDMHMDTGNAQYMEEFFEQVEEIRGSVDILANNVEEVKKKHSAILSNPVNDQKTKEEL DELMAVIKRAANKVRGKLKLIENAIDHDEQGAGNADLRIRKTQHSTLSRRFVEVMTDYNKTQTDYRERCKGRIQRQLD LAGKQVGDEDLEEMIESGNP GVFTQGIITDTQQAKQTLADIEARHNDIMKLESSIRELHDMFMDMAMLVESQGEMVDR IEYNVEHAKEFVDRAVADTKKAVQYQSKARRKKICILVTGVILITGLIFILFYAKVL

\section{Figure 11. Sequence coverage of Unc18 and Unc64}

The sequence coverage (peptides detected after trypsin digestion) of the sequences of Unc18 and Unc64 from the mass-spectrometry studies amount to $90.0 \%$ for Unc18 and resp. $72.5 \%$ in Unc64, which are in accordance to the levels allowed for structural analysis and means that on average the sequence covered in every experiment corresponds to $90.0 \%$ in Unc18 and $72.5 \%$ in Unc64. In black are regions that cannot be identified during the Mass-spec studies, if modifications take place in these regions it cannot be recorded.

Altogether the foot-printing yielded three regions that were protected upon complex formation, the first one (peptide 4) corresponds to the region between residues 4 - 10 in the Unc64 sequence and thus probably spans the $\mathrm{N}$ peptide of Unc64 (Fig. 12). The presence of this region on the list was to be 
expected as this corresponds to a crucial region of interaction, this has been proven in studies in where the removal of this region affect the binding of Syntaxin1a to Munc18a, and in our experiments, Unc64 to Unc18. The corresponding binding region in Unc18 aligns well with the hydrophobic pocket in in Munc18, and when mapped on the structure (PDB: 3C98) it can be found facing the N-peptide (data not shown). These results are in accordance with previously published data (Johnson, Ferdek et al. 2009) (Burkhardt, Hattendorf et al. 2008).

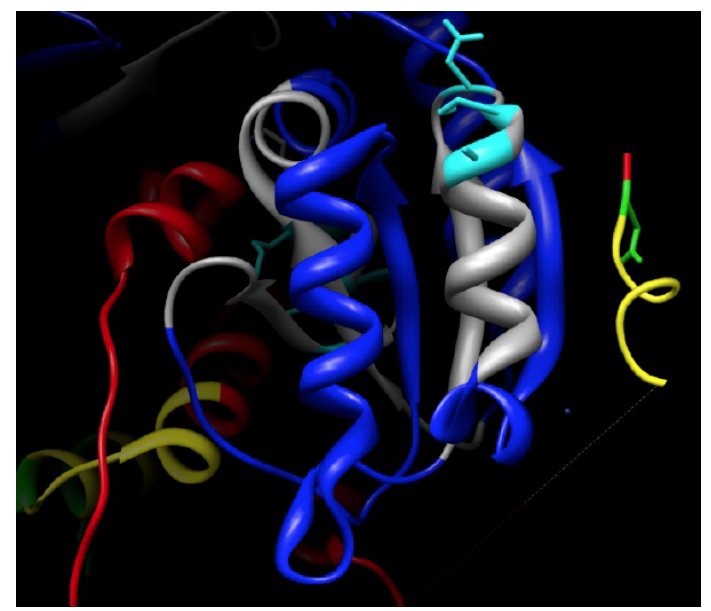

Figure 12. Proposed interaction of Unc18 and Unc64 based on the modification found in peptide 4.

Regions of interaction between Unc18 (Blue) and the Unc64 N-peptide (Yellow) according to Mass-spec analysis of modified side chains, due to the high degree of sequence similarity the results obtained from the Mass-spectrometry analysis can be localized into regions of the already solved structure (3C98) of the mammalian Munc18 and Syntaxin1a, Peptide 4 contains a modified aspartic acid (D) when un-bound but protected upon complex formation.

Two other peptides that were found to be protected of modification upon complex formation were peptide 13, covering residues 97-115 of Unc64 (Fig. $13 \mathrm{~A}$ ), that based on sequence alignment the peptide could be assigned to a region of syntaxin1a that binds into the groove of the horseshoe that is typical for SM proteins, and the other was peptide 15 covering residues 191-199 on the Unc64 sequence. The protection of the peptides 4 and 13 are in agreement with a binding mode in which the N-peptide and the closed conformation of syntaxin bind to the SM protein as has been reported for the mammalian homologous (Burkhardt, Hattendorf et al. 2008). It also supports the ITC results that have 
been summarized in table 1 . Interestingly, peptide 15 does not correspond to an area of syntaxin that has previously been reported to interact with the SM proteins and draws the attention to a novel region that might be important for our understanding of the interaction between Unc18 and Unc64 and maybe for SM proteins and syntaxin in general (Fig. 13B).

A

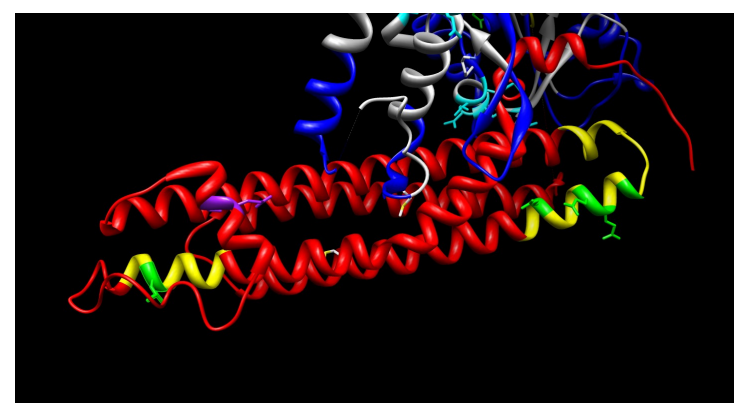

B

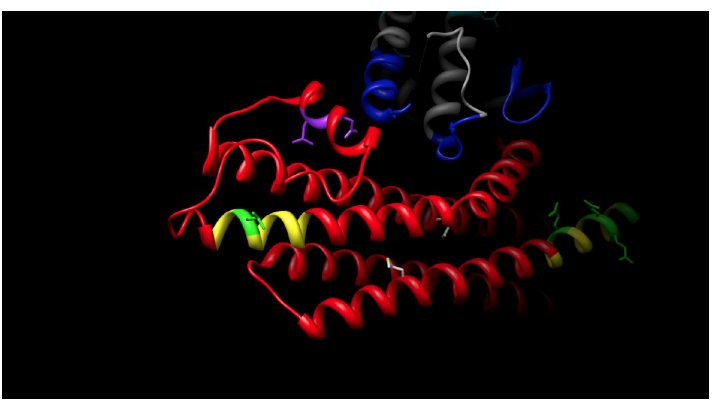

Figure 13. Modified regions on the Unc64 sequence upon complex formation

Peptide 13 can be localized to a linker region found in structure 3C98 in the groove of Munc18 (Fig A highlighted yellow) and would be expected to be protected from modification upon complex formation if the binding mode is similar to the one exhibit in Munc18/Syntaxin, while peptide 15 is near the loop that connects the Hc helix and the SNARE motif and represents a novel region that might elucidate an uncovered regulatory mechanism (Fig B).

\subsection{Peptide 15 reveals a highly conserved region adjacent to layer -7 and makes a physical interaction with the linker helix}

Peptide 15 corresponds to a region between a regulatory loop that connects Hc-helix and the SNARE motif of Syntaxin. This linker region has been shown to be important in the regulation of the propensity of the Qa SNARE to take part in the SNARE complex formation (Burkhardt, Hattendorf et al. 2008) (Johnson, Ferdek et al. 2009) (Dulubova, Sugita et al. 1999; Gerber, Rah et al. 2008). Upon closer inspection, this loop revealed some interesting features, starting with the high number of hydrophobic residues assembled in the area (Fig. 14A-C). When this area was compared to the other members of the SM family which structures are solved, it was observed that the high degree of hydrophobicity is present in all of them; 36\% in Syntaxin 1a (PDB:3C98), 51\% in Sso1p (PDB:1FIO), 62\% in the Syntaxin 1 of Monosiga brevicollis (PDB:2XHE). 
A

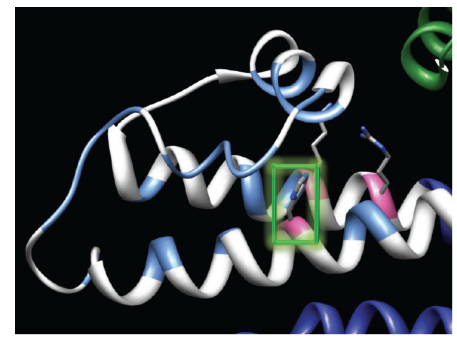

$\mathrm{B}$

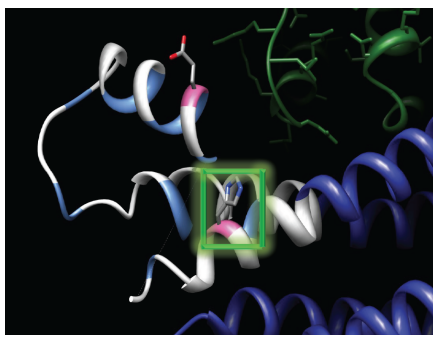

C

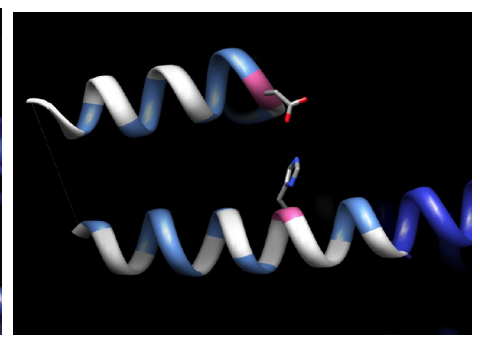

Figure 14. Peptide 15 reveals the dynamics of a region near a loop shown to be important in SNARE complex regulation.

The modification of peptide revealed a region that gets protected upon complex formation, this peptide spans a small region beyond layer -7 of the conserved SNARE motif and after the loop that connects the Hc helix with the SNARE motif and that has been shown that mutations in it renders an open conformation in Syntaxin (LE "open" mutant). Peptide 15 represents an area adjacent to a highly conserved histidine (H199) and contains an arginine (R198), this Histidine is found to interact directly with the loop in both the complex crystal structures of the Rattus Norvegicus Syntaxin 1a (His200) (A), the complexed Syntaxin in monosyga bevicollis (H216) (B) and in the un-complex Sso1p from Saccaromyces cereviseae (His197)(C). The areas that are compared are equivalent in length and highlighted in white, the hydrophobic residues in the region are blue and the interconnecting residues in purple.

Peptide 15 covers an area that is adjacent to a histidine at position 199 that can be observed in crystal structure of the homologue Syntaxins to make a physical interaction with a region that links the Hc helix of the Habc domain to the SNARE motif (Fig. 14A-C), this linker helix in Syntaxin1a from Rattus norvericus binds to a histidine in position 200, while in the Syntaxin of Monosiga brevicollis the analogous histidine is found in position 216, it is worthwhile to point out that the two Syntaxins (Fig. 14A and B) show an interaction that can only be seen in complex with their corresponding SM proteins while Sso1p Histidine 197 seem to be fully capable to create an interaction on its own (Fig. 14C).

In order to assess the degree of conservation in and around the region corresponding to peptide 15 more closely, a sequence alignment of all Qa.IV SNAREs (Kloepper, Kienle et al. 2007) was generated with the program MUSCLE, this alignment consisted in 902 sequences that were truncated leaving 8 residues to the $\mathrm{N}$-terminal region of the Histidine and 27 to the $\mathrm{C}$-terminal (well into the SNARE motif), I set this limit to include the glutamine of layer "0" in the 
SNARE motif and enough to cover the helix in which the SNARE motif is found before it is transformed into the binding loop that connects to the Hc helix. The alignment was kindly provided by Dr Kloepper.

As this histidine seems to interact with the helix a visualization of the degree of conservation was done of the alignment with the software "Weblogo" which creates a graphical representation of the conservation of a residue by a difference in height, the more conserved the residue is the taller the letter is (Crooks, Hon et al. 2004).

The alignment of the 905 syntaxins (Qa-SNAREs) shows a high degree of conservation for that particular histidine and in addition revealed a directly adjacent arginine that is even more conserved throughout all secretory syntaxins (R198 in Unc64). Although the arginine is not absolutely conserved, the charge seems to have been kept preserved and can be compared to the glutamine in layer “0” in Syntaxins (Fig. 15) (Fasshauer, Sutton et al. 1998).

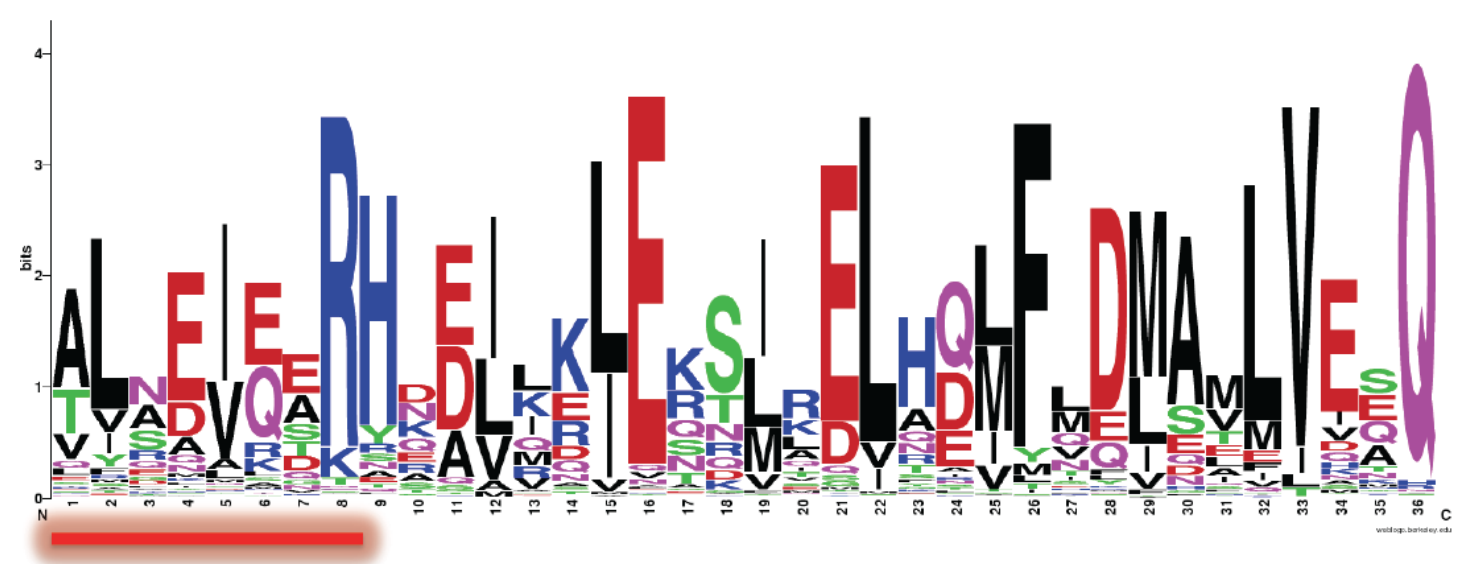

Figure 15. Peptide 15 reveals a conserved region near a loop that connects the SNARE motif with Hc helix.

A representation of the degree of conservation of the region around peptide 15 (underlined in red) reveals a Histidine that has been shown to interact directly physically with the loop region connecting the Hc helix and the SNARE motif, as reported on the crystal structures 1FIO, 3C98 and 2XHE. The Mass-spectrometry results did also unveil an arginine that has an even higher degree of conservation, and can be compared to the Glutamine in position 36 (pink) in the figure, this Glutamine has been used to create the new SNARE nomenclature. The numbering is based on the position of the residue in the sequence analyzed. 


\subsection{Study of the Sec1p interaction to the Qa SNARE Sso1p and the yeast SNARE complex}

The details regarding mechanism with which Sec1p interacts with the components of the SNARE complex and helps promote secretion in S. cerevisiae has been at the center of several studies (Brummer, Kivinen et al. 2001) (Carr, Grote et al. 1999) (Hashizume, Cheng et al. 2009) (Weber-Boyvat, Aro et al. 2011) (Weber, Chernov et al. 2010) (Aalto, Jantti et al. 1997; Scott, Van Komen et al. 2004) (Togneri, Cheng et al. 2006).

Most insights on the function of Sec1 were gained by genetic screens. In addition, first attempts to study the underlying protein-protein-interactions have been reported. In these studies the interaction patterns were studied by means of pull-downs and western blots of endogenous proteins, very probably because it proved difficult to express the SM protein Sec1 in E. coli. In those studies it was reported that Sec1 mainly binds to the assembled SNARE complex rather than to the cognate Qa Syntaxin Sso1p (Togneri, Cheng et al. 2006) (Scott, Van Komen et al. 2004; Hashizume, Cheng et al. 2009)

In order to obtain deeper insights I attempted to study the mode of Sec1 SNARE interaction by using the isolated components obtained by recombinant means (Fig. 16).

A crucial component in these studies is $\operatorname{Sec} 1 \mathrm{p}$, as it is notoriously hard to work with, it has been a limiting factor for doing in vitro studies. As this was important a lot of effort was put on developing a protocol to produce Sec1p by recombinant means. This goal was reached and I managed to produce it in large enough amounts but in a diluted form, the concentration problem remains and we can only limit ourselves to a few methods to study it. 


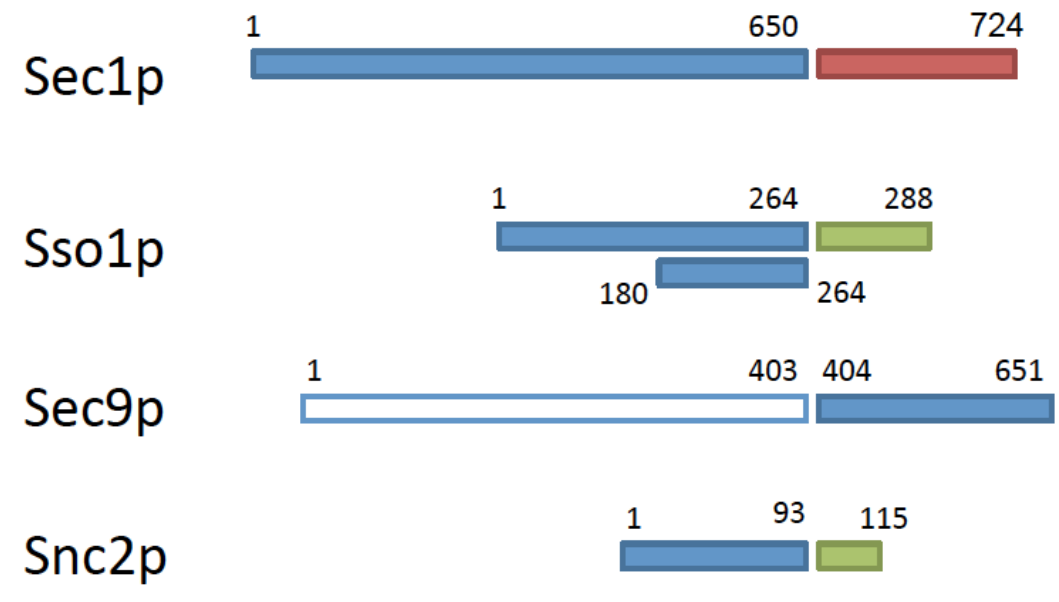

Transmembrane domain
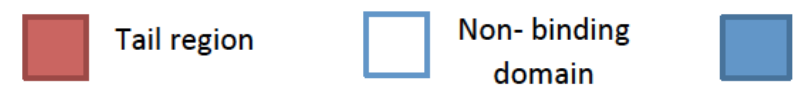

Binding region used in studies

Figure 16. Schematic diagram of the constructs used in the Sec1p interaction studies.

The constructs used in the interaction experiments in solution are with the regions that have been previously reported to interact. The Sec1p sequence is in full (1-724) and includes the C-terminal region that contains a sequence rich in basic residues (red section). Sso1p is used in two versions; one that consist the cytosolic region and does not contain the transmembrane part (green section) and one that is composed of the SNARE motif only (180-264). The Sec9p construct consists of the region that has been reported to be involved in SNARE complex formation (404-651), while for Snc2p only the cytosolic region is used (1-93) and the transmembrane region (green section) is not included.

\subsection{Sec1p does not bind to the Qa SNARE Sso1p, the binary Sso/Sec9 complex nor the ternary SNARE complex in vitro}

In order to investigate whether Sec1p binds to Sso1 or to SNARE complexes in vitro, pre-bound GST-Sso1 was mixed stoichiometrically with its partner SNAREs and with Sec1p and let bind over the course of two hours at $4^{\circ} \mathrm{C}$ before they were "pulled-out" through the GST tag of Sso1 (Scott, Van Komen et al. 2004) (Togneri, Cheng et al. 2006). GST-Sso1 was pulled down together with Sec9 (i.e. forming the binary Sso/Sec9 complex) (Fig. 17 lane 4) or with Sec9 and Snc1 (i.e. forming the ternary SNARE complex) (Fig. 17 lane 5) but not together with Sec1 (Fig. 17 lane 3-5), even if the components were left to bind over night (Fig. 17 lane 7). Surprisingly, as it is in stark contrast to the findings reported in 
the literature, in fact, the vast majority of Sec1p can be seen in the flow through in all cases (fig 16 lane 6 from SNARE complex assembly), and any amount of Sec1p that can be pulled out can be accredited to unspecific or low affinity binding.

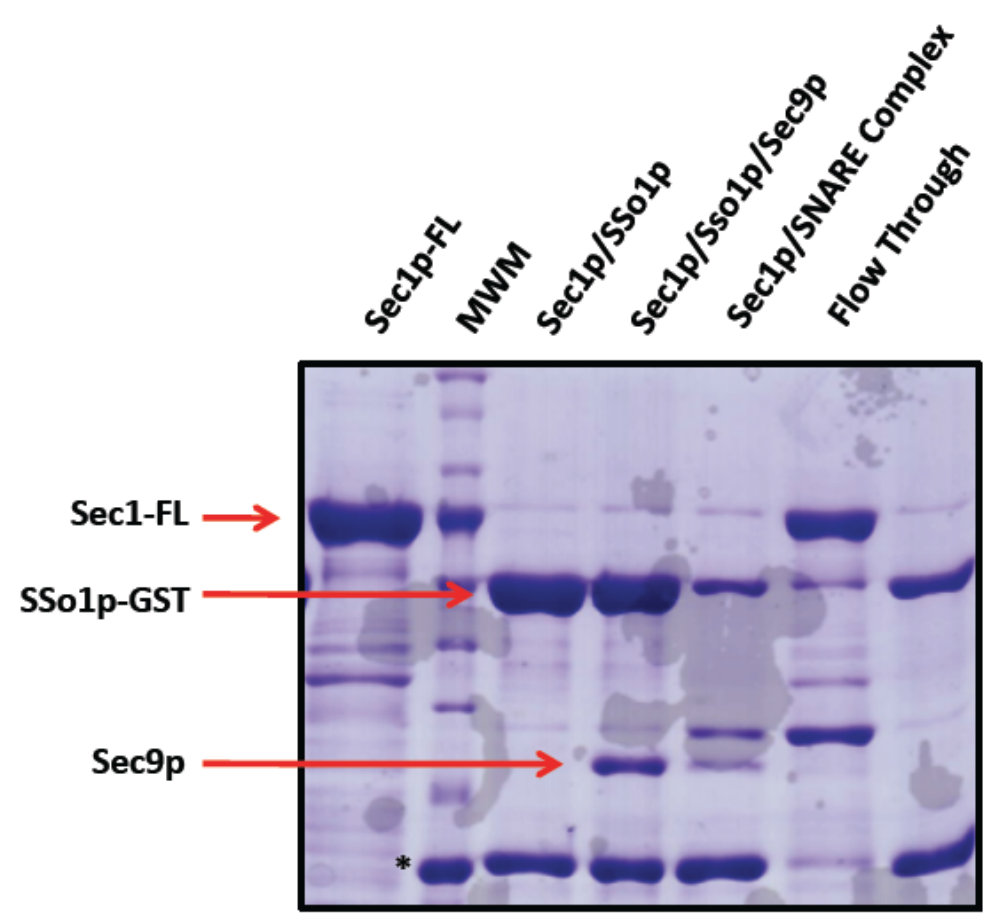

Figure 17. Interaction studies of Sec1p to the SNARE complex components.

According to previous studies, Sec1p binds exclusively to the assembled SNARE complex both in vivo and in vitro. The interaction of Sec1p was put to test by means of GST pull-down as the components were mixed and left to bind for two hours before analyzed by SDS-PAGE electrophoresis. At first the ability of Sso1p-GST to pull-down Sec1p (lane 3) was tested with the result that no interaction was observed, this held true as the components were incubated overnight (lane 7). Sec1p did not show an affinity towards the binary complex formed by Sso1p-GST and Sec9p, as was expected (lane 3). One surprising result was that Sec1p doesn't seem to bind to the SNARE complex (Sso1p-GST, Sec9p, Snc2p) either but is found in the Flow through (lane 5).

In order to rule out that the failure to observe binding of $\operatorname{Sec} 1 \mathrm{p}$ to the SNARE complex is due to the nature of the assay used, a different in vitro assembly assay was performed. The assembly reaction consisted in combining the different untagged SNARE components in equimolar amounts and let interact for two hours at $4^{\circ} \mathrm{C}$ before the reaction was analyzed, as the yeast SNARE complex is not SDS resistant, the reactions were analyzed by native gel electrophoresis. 
Two different types SNARE complexes were assembled in theses experiments: one in which only the SNARE motif of Sso1 was used. The other one was assembled using a construct containing the entire cytoplasmic domain of Sso, i.e. it contained the inhibitory Habc domain.

Both variants of Sso1 were able to form the binary Sso1/Sec9 complex as well as the ternary SNARE complex (Fig. 18A). In order to test whether Sec1 has any effect on the rate of SNARE complex formation, SNARE assembly was carried out in the presence of excess amounts of Sec1 as well. No effect of Sec1 on the assembly of the binary complex (Fig. 18 B lane 2 vs lane 4) or ternary SNARE complex (Fig. 18B lane 1 vs lane 3) was observed, however.

A

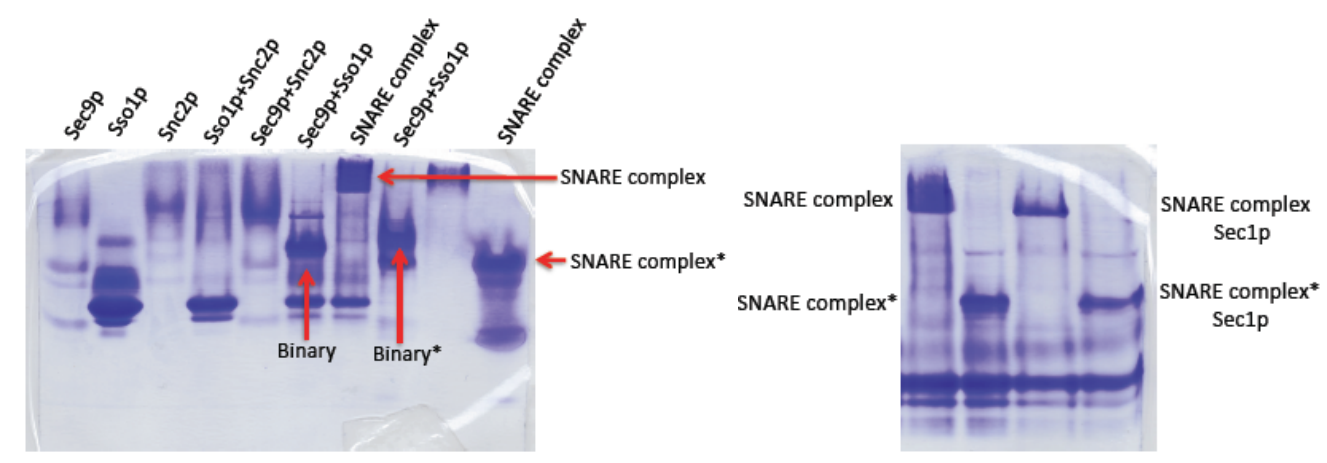

Figure 18. Effect of Sec1p on SNARE complex formation.

The effect that Sec1p was further studied with soluble components were mixed and let to interact for 2 hours before analyzed by native gel electrophoresis, this was necessary as the yeast SNARE complex is not SDS resistant. The results obtained from these studies show that the components are in full capacity to interact with one another and form the binary complex (Gel A lane 6) and the SNARE complex (Gel A lane7) when Sso1p containing the Habc domain is added to the reaction, as well as when the binary and SNARE complex are assembled with a Sso2p construct that lacks the Habc domain and is composed of the SNARE motif only (Lane A8* and Lane 10*). The reactions assembled with the SNARE motif only are marked with an asterisk $\left(^{*}\right)$. 


\subsection{Sec1p contains a positively charged region on its C-terminal region that can assist Sec1p to localize to the membrane}

Sec1p contains a positively charged tail region (residues 651 and 724; estimated pI of 10.1). This tail region is only present in most secretory SM proteins of fungi and thus this trait seems to have been acquired during evolution of fungi. As Sec1p exerts its function at the plasma membrane, it was of interest to investigate whether this positively charged tail might be a membranebinding device that could assist in localizing Sec1p to the plasma membrane and promotes the interaction with Sso1p. In order to answer this question an assay that consist in allowing 40nm liposomes (Fig. 19B) to move throughout a density gradient created through high speed centrifugation was used, the rational is that if the protein binds to the liposomal membrane it would move through the gradient in complex with it (Fig. 19A).

A

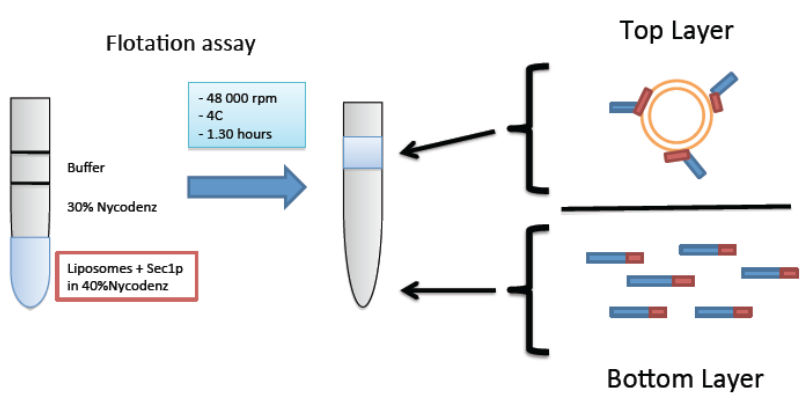

B

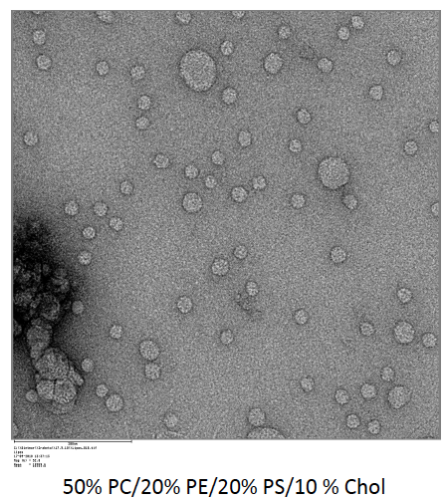

Figue 19. Sec1p contains a potential membrane binding region.

In order to monitor the ability of Sec1p to membranes, liposomes were created by mixing a lipid solution with 50\% PC, 20\% PE, 20\% PS/10\% Cholesterol and then taken though a Fast Desalting PC 3.2/10 column were the lipids assemble spontaneously into $40 \mathrm{~nm}$ liposomes (B). The floatation assay (A) consists in the mixing of liposomes and protein in a solution that containins $40 \%$ Nycodenz, this mixture is put at the bottom of a tube upon which a solution composed 30\% Nycodenz I carefully laid. The upper layer consist in a buffer solution (20mM HEPES, $250 \mathrm{mM} \mathrm{NaCl}, 1 \mathrm{mM}$ DTT pH8.0), the samples are centrifuged at $4^{\circ} \mathrm{C}$ for one and a half hours at $48000 \mathrm{rpm}$. 


\subsection{Study of the Sec1p interaction to Sso1p and the yeast SNARE complex in the presence of a lipid membrane}

In order to assess whether the interaction between Sec1p and its cognate Sso1p or the SNARE complex requires the presence of a membrane, a Sso1p construct containing the trans-membrane region (Fig. 16) (Sso1pTM) was produced. The purified protein was incorporated into liposomes and a floatation assay as described above was performed (Fig. 19A).

First it was tested whether Sec1p binds to Sso1pTM alone and or the SNARE complex (Sso1p/Sec9) in the presence of a membrane. After centrifugation both the most upper layer and the bottom layer were analyzed by SDS-PAGE. From these studies it can be seen that Sso1pTM is incorporated successfully into the membrane of the liposomes (Fig. 20 Gel A lane number upper fraction 1-3 as well as Fig. 20 Gel B lanes number 1 and 3 upper fraction) and that the binary complex can be formed (Fig. 20 Gel A lane nr 3), although the incorporation of Sso1pTM and the binary complex is formed it is very hard to conclude that the presence of Sec1p on the top layer is due to the interaction to Sso1p as Sec1p is found at the top layer in the absence of Sso1p (Fig. 20 Gel A lane nr 4 and Gel B lane nr 2) and could be through the C-terminal tail rather than an interaction with Sso1p.

In contrast it is very clear that Sec9p binds to the liposome only through Sso1pTM as it cannot be found on the upper fraction on its own (Fig. 20 Gel B lane 2 upper fraction) but rather the bottom fraction (Fig. 20 Gel B lane nr 2 and 4 bottom fraction), or in the presence of Sec1p, meaning that it does not bind unspecific to the lipid layer nor to Sec1p. 
A

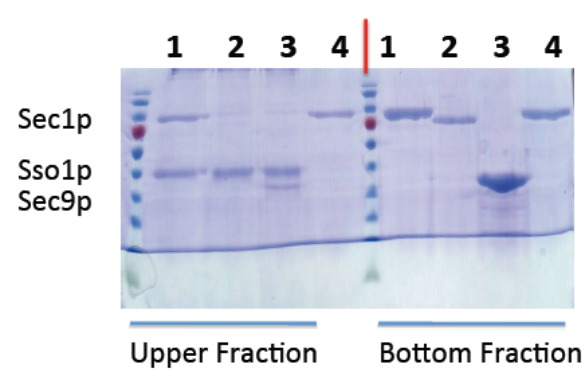

B

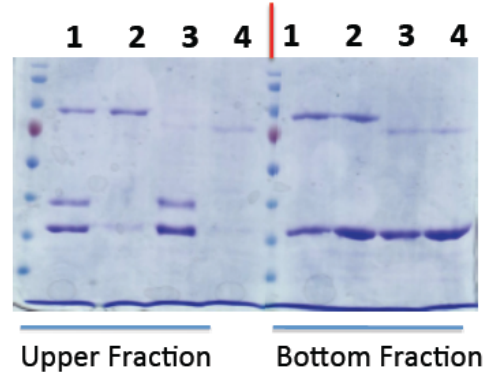

Figure 20. The interaction of Sec1p to the SNARE complex in the presence of a membrane.

As Sec1p is found in the vicinity of the membrane at the bud neck and the growing bud in proximity of the SNARE complex components it can be argued that the reported interaction of Sec1p to the assembled SNARE complex takes place only in the presence of the membrane. Gel A shows the interaction between Sec1p and Sso1pTM (Containing a transmembrane region) that has been incorporated to the membrane in lane number 1 (upper fraction), while lane 2 (upper fraction) contains Sso1pTM incorporated on the membrane and a control protein to assess the specificity of the interactions to Sso1p. Lanes 3 (upper fraction) contains Sec9p and Sso1pTM (binary complex), while lane 4 (upper fraction) contains Sec1p and no Sso1pTM respectively. In gel B the question of whether Sec1p binds to the SNARE complex in the presence of the liposomal membrane was studied as the SNARE complex was assembled on the membrane and mixed with Sec1p (Gel B lane 1 Upper fraction) or a control protein (Gel B lane 3 Upper fraction) the specificity of this interaction was controlled by mixing Sec1p and Sec9p with the liposomes in the absence of Sso1pTM (Gel B lane 2 Upper fraction) and with a control protein with Sec9p with the liposomes in the absence of Sso1pTM (Gel B lane 4 Upper fraction).

In conclusion it can be said that no interaction was detected between Sec1p with any of our methods, as it cannot be pulled down with a GST-tagged Sso1p construct in any of the different combinations. It cannot be seen to affect the SNARE complex assembly, as the native gel analysis shows, while floatation assays show that this holds even in the presence of a membrane.

\subsection{Engineering of a Sec1p construct with increased solubility}

That Sec1p is essential for secretion has been shown in several studies (Brummer, Kivinen et al. 2001; Scott, Van Komen et al. 2004; Togneri, Cheng et al. 2006; Hashizume, Cheng et al. 2009; Weber, Chernov et al. 2010; WeberBoyvat, Aro et al. 2011). However, as outlined above so far no proper dissection 
of the interaction between Sec1p and the different components of the SNARE machinery has been achieved in vitro (Scott, Van Komen et al. 2004) (Togneri, Cheng et al. 2006). Very likely this is due to the fact that it is difficult to produce Sec1p by recombinant means in E. coli, this seems to be the case for the Sec1p expressed in $S$. cerevisiae as the results obtained do not included other methods then Pull-dows hinting that the concentration problem has not been overcome, in addition no other systems have been reported in the literature (Togneri, Cheng et al. 2006) (Hashizume, Cheng et al. 2009).

Moreover, a construct encoding for a shorter version that lacks the Cterminal positive tail was generated. However, this construct did not increase the yield. This was further complicated by the fact that Sec1p has the propensity to aggregate after purification. This made it challenging to reach protein concentrations required for biophysical approaches like ITC. The maximal concentration of purified Sec1p that was reached was about $2 \mathrm{mg} / \mathrm{ml}$.

To increase the yield of Sec1p produced in E. coli I used several different methods (data not shown) for expression and purification including truncation of the positively charged C-terminus (1-639), and a region that is predicted to be disordered between 543-559 (construct 1-543). However promising these constructs seemed they precipitated completely upon lysis of the cells, why the full length Sec1p is produced more successfully (enough to carry out the experiments mentioned above) is probably due to the tail, it has been showed that a highly charged tag can help increase the solubility of recombinant proteins, this because a highly charged region can inhibit the aggregation process through electrostatic repulsion. This repulsive force can give the protein the opportunity to stay in solution long enough to fold properly (Zhang, Chen et al. 2004).

Other strategies that I tried to increase the solubility of the full length Sec1p included the increase of chaperons by exposing the bacteria to both heat shock and ethanol shock (Oganesyan, Ankoudinova et al. 2007), as well as by adding Betaine and Sorbitol (Sandee, Tungpradabkul et al. 2005). This in 
combination with different IPTG concentrations (ranging from 0.01-0.5mM), expression temperatures (ranging from $18^{\circ} \mathrm{C}$ to $30^{\circ} \mathrm{C}$ ) as well as expression strains (Origami, BL21 (DE3) pLyss).

As all the attempts to increase the solubility of Sec1p and the truncated constructs failed I decided to go back to the sequence and tried to see if it would reveal new details that could be responsible for promoting the aggregation. In order to engineer a construct with increased solubility a close look was taken at the Sec1p sequence. The engineering of Sec1p is difficult because of low sequence similarity between Sec1p and the other SM proteins for which more detailed mechanistic and structural information is available, so instead of looking at similarities at the sequence level, a look at the secondary structure level was done.

The first step taken was to make a secondary structure prediction of Munc18 with PSIPRED (Buchan, Ward et al.), this prediction was later validated by comparing it to the secondary structure observed in the solved crystal structure (3C98) and from this it could be concluded that the prediction was highly accurate. The second step was to create a prediction of Sec1p and once the prediction for Sec1p was done a comparison was done against the prediction of Munc18. The aligned sequences show that even though the sequences are not highly conserved, the secondary structures are very similar (Appendix 1). This rather complicated approached allowed me to answer the question of whether there was some information available inside the poor alignment, indeed I could orient myself with the help of the secondary structure elements and verify that they are placed in the right order. Through this approach a short stretch that is part of a predicted helix close to the N-peptide binding site was identified (Fig. 21 region $\mathrm{nr} 2$ ) and although I cannot rule out that it is an important region, as it has been proposed that Mso1p binds in the vicinity (Weber, Chernov et al. 2010) its removal could potentially increased the solubility. This region is badly conserved in other SM proteins (data not shown). With this in mind a construct that lacks both this peptide and the C-terminal tail was created and the expression was tested. 


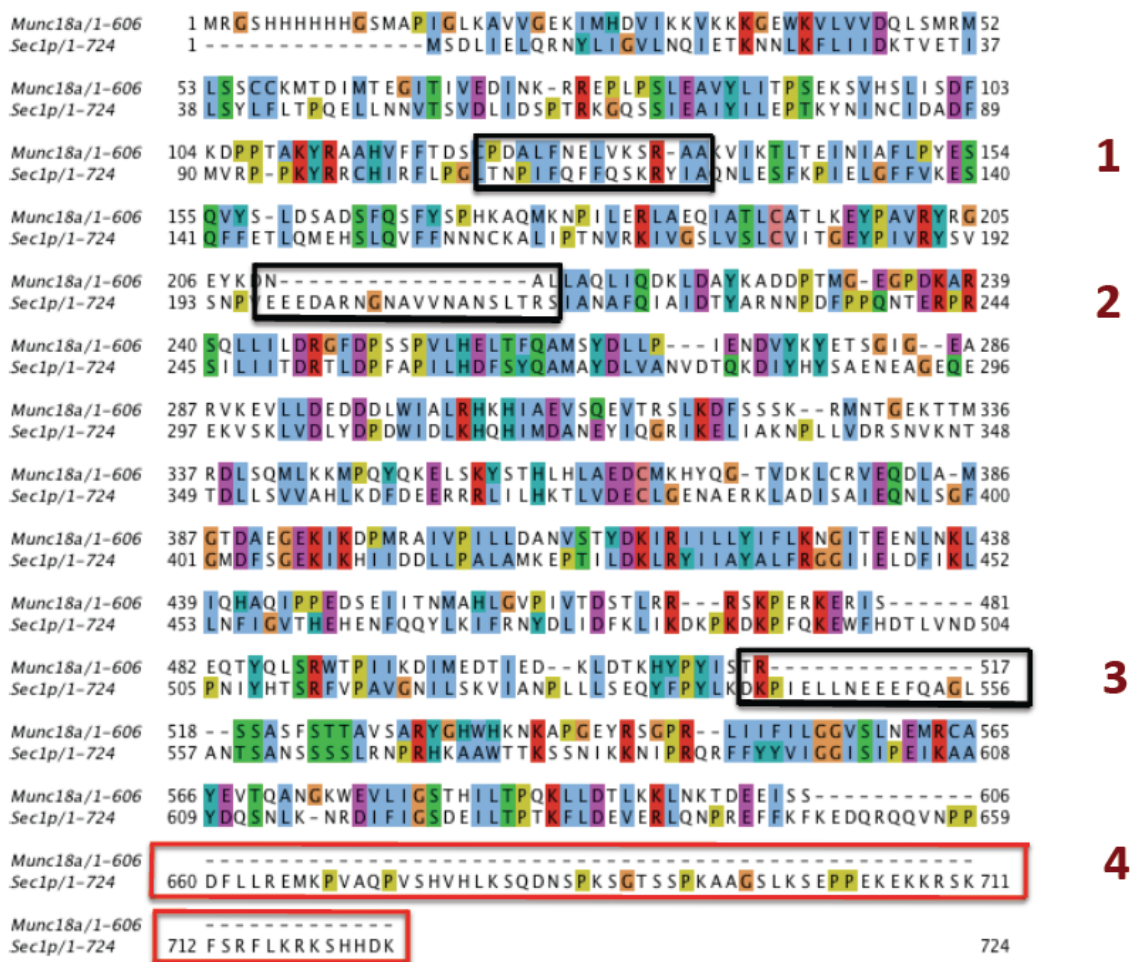

Figure 21. Sec1p/Munc18a alignment

In order to obtain be able to compare Sec1p with Munc18a, an alignment was prepared with MUSCLE, this shows 4 interesting regions: region number 1 shows that there is a potential $\mathrm{N}$-peptide binding site in Sec1p, region number 2 shows an extra region that consist of 17 residues. Region 3 shows yet another region that contains 16 extra residues and finally region 4 shows the positively charged C-terminal tail of Sec1p.

Indeed, the shortening of the loop (Fig. 23A) helped to increase the solubility of the protein (Fig. 23B), although further optimization is required in order to keep it in solution for longer periods of time. Another construct created for studying the function of Sec1p consist on the region that corresponds to Domain 1 in other SM proteins such as Munc18 (Fig. 23D), this region could relieve any restrains or blockage that might inhibit the binding of Sso1p and on top of that, and furthermore contains the potential N-peptide binding site. 


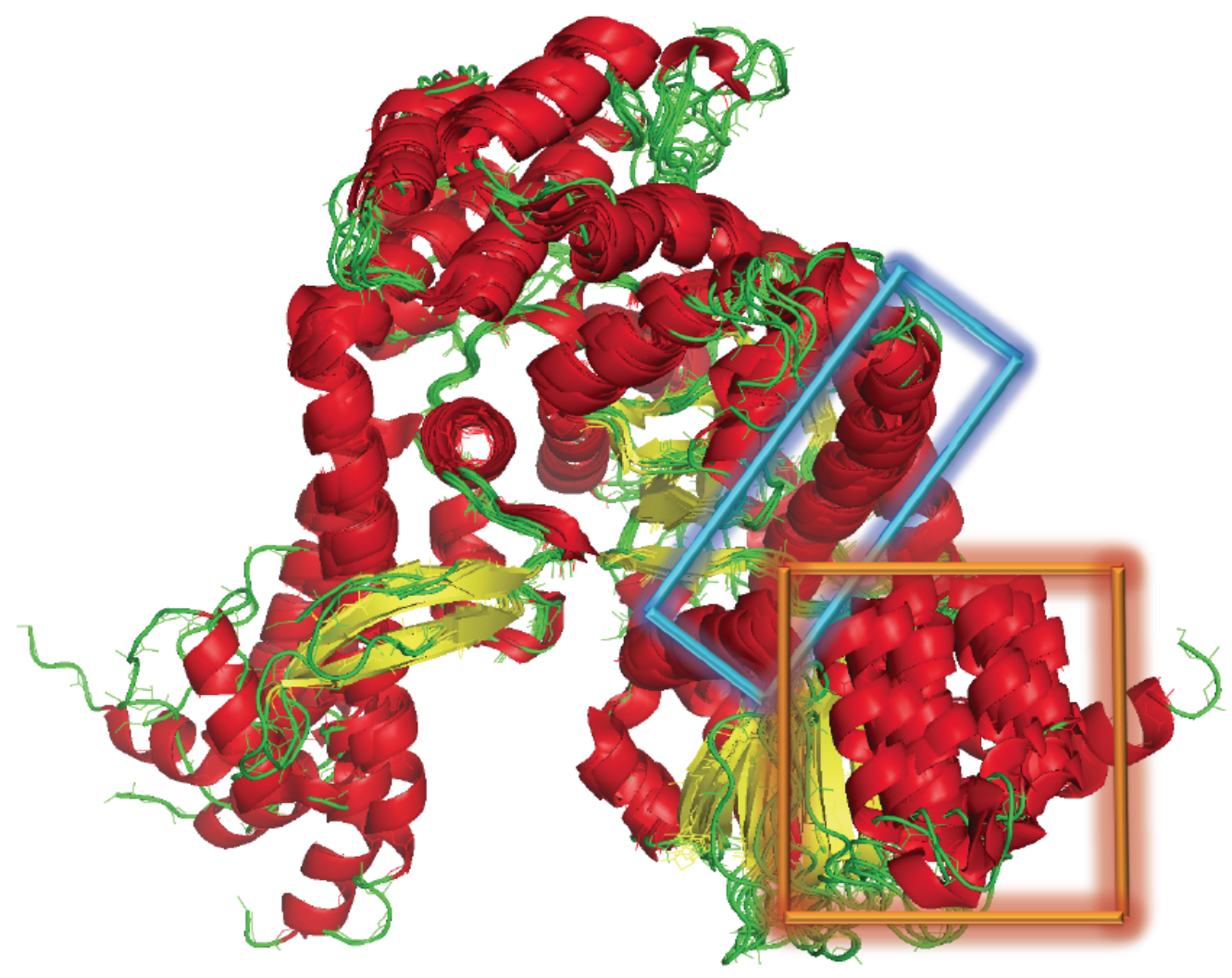

Figure 22. Superimposition of all the SM proteins.

A superimposition of all the solved crystal structures (3C98, 1FVF, 2XHE, 1EPU, 2PJX, 3PUJ, 3PUK) shows an similarity in the overall fold of the members of the family at the same time points at the importance of the extended helix found in the alignment and marked as region 2 in Sec1p is shown here in the blue frame, assuming that the secondary structure prediction indicates a similar fold, this would show that the extra region is close to the $\mathrm{N}$-peptide binding site (red frame), this area corresponds to an area important for the binding of Mso1p that has been proposed to replace the N-peptide (Weber, Chernov et al. 2010).

Another protocol that I emphasized on was for the co-expression and purification of the Sec1p/Mso1p complex, as Mso1p has proven to be crucial component for secretion in yeast (Weber, Chernov et al. 2010) (Weber-Boyvat, Aro et al. 2011) (Aalto, Jantti et al. 1997) (Knop, Miller et al. 2005). This protocol is based on the co-expression of a GST-tagged Mso1p construct and a His-tagged Sec1p construct that can be co-purified with by means of Glutathion Agarose beads (Fig. 23C lane1), and by means of Ni-NTA beads after cleavage of the GSTtag (Fig. 23C lane2). 
A

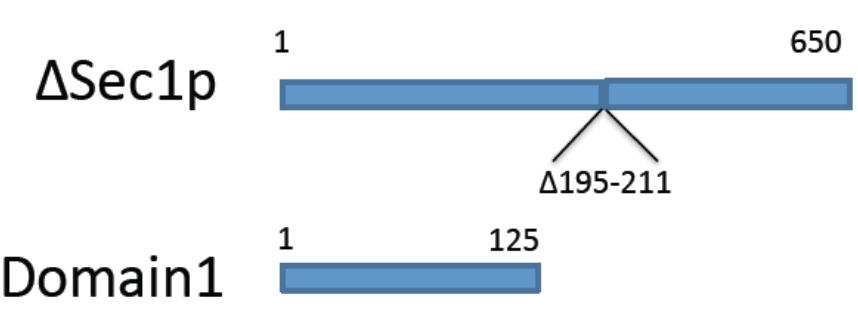

B

C

D

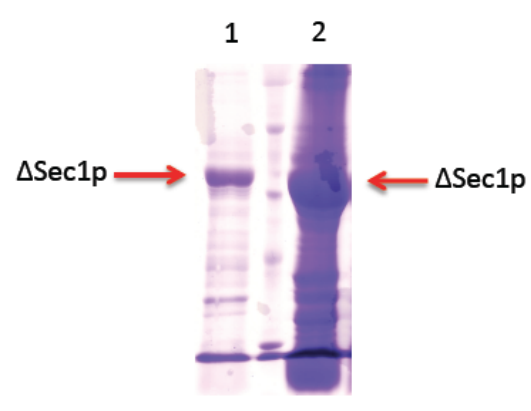

12
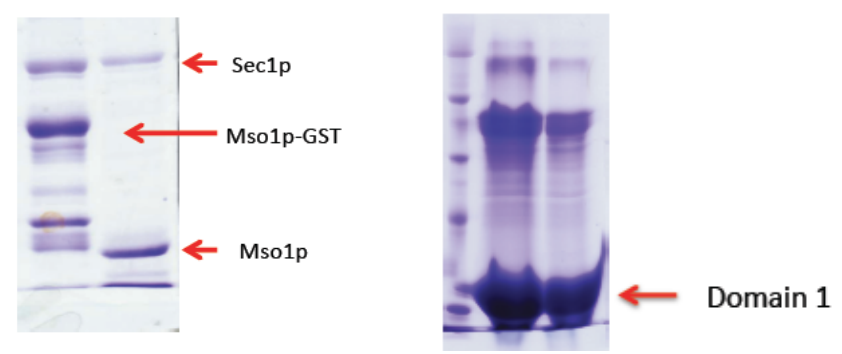

Figure 23. Novel constructs to study the binding of Sec1p to the SNARE components.

As Sec1p has proven to be a very hard protein to work with it requires the development of alternative constructs to overcome the difficulties shown by Sec1p, which includes instability and an unknown alternative-binding mode. For this reason a construct composed of what would constitute domain 1 in the SM proteins (residue 125) was engineered and expression tested and purified by Ni-NTA beads (D), at the same time a construct of Sec1p lacking both the C-terminal domain (1-650) and the region consisting the extended loop (195-211) was engineered $(\Delta \operatorname{Sec} 1 \mathrm{p})$ and the expression tested and purified by means of $\mathrm{Ni}$ NTA beads (fig 17B in lane 1 the boiled E.coli after overnight expression while lane 2 shows the band after purification with Ni-NTA beads). Both these new constructs will provide new ways to analyze the mechanism of action of Sec1p. 


\section{Discussion}

Cellular secretion, whether it takes place in the neuron during synaptic transmission in, in beta cells during the release of the peptide hormone insulin, or serves to deliver newly synthesized membrane proteins, lipids or material to build a cell wall to the cell surface, requires the fuse of vesicles to the plasma membrane in order to release their cargo to the extracellular space. The mechanism with which membranes fuse require a defined set of proteins and at the core of this machinery lies the so-called SNARE proteins, that have been accredited with providing the system with the raw mechanical force to overcome the repulsive barrier and fuse the membranes. In the mammalian synapse the group of SNAREs used are Syntaxin1a (Qa), SNAP-25 (Qb and Qc) and Synaptobrevin (R-SNARE), while in the closely related homologues involved in neuronal secretion in C. elegance are Unc64 (Qa), Ric4 (Qb and Qc) and Snb1 (RSNARE). In baker's yeast, a more distant homologue to the mammalian system, the fusion machinery responsible for secretion is composed of Sso1p (Qa), Sec9p (Qb and Qc) and Snc2p (R-SNARE).

The regulation of the SNARE complex assembly is a key mechanism believed to be provided by the SM family of proteins, this has been shown through genetic studies that demonstrated that its different family members are essential for various vesicle trafficking steps in the cell. It has been proposed that these proteins act through an interaction to their cognate Qa-SNAREs in three different ways: through the N-peptide of the Syntaxin (mode 1), to the closed Syntaxin (mode 2), or to the assembled SNARE complex (mode 3). According to this idea the interaction modes through the N-peptide and to the SNARE complex were believed to represent different steps in which the SM proteins promotes the formation of the SNARE complex between the two fusing membranes. By contrast, binding through the four-helix bundle (closed syntaxin) was claimed to be a peculiarity of the SM protein involved in neuronal exocytosis in vertebrates. 
In this mode Munc18-1 was thought to sequester syntaxin rather than preparing it for SNARE complex formation.

Recently, the SM protein Munc18-1 has been shown to interact with Syntaxin 1a through a combination of both the N-peptide and the closed fourhelix bundle (mode1 and mode 2). The same binding mode was found for Munc18 of the choanoflagellate Monosiga brevicollis. Both structures show that Munc18 binds simultaneously to the N-peptide region and to the closed conformation of syntaxin. It was also reported that other SM protein types, Vps45 (Burkhardt, Hattendorf et al. 2008) (Furgason, MacDonald et al. 2009) and Sly1 (Demircioglu 2011), that act in other trafficking steps interact with two bindings sites as well. These findings show that the view that mammalian Munc18-1 has a special role cannot be maintained. Still, a unifying model of SM protein function has not been developed yet, as many published observations are difficult to reconcile. Aside from the SM protein Vps33 that is part of a larger protein complex, the so-called HOPS complex, and that has not been studied in much detail on a molecular level, the yeast secretory SM protein Sec1 seems to be difficult to place into the picture. Sec1, in contrast to most other SM proteins, had been reported not to bind to its cognate syntaxin Sso1p but only to the assembled SNARE complex.

In the current study it has been in our interest to investigate whether a more general binding mode could be found by comparing different secretory SM proteins in detail using biochemical, biophysical and structural approaches. In particular, we wanted to scrutinize the binding mode of Sec1 to the assembled SNARE complex in more detail. For comparison, the neuronal secretory proteins of the nematode C. elegans were studied. At the beginning of my work it seemed possible that the binding mode of Sec1 might represent a general reaction intermediate of the interaction of SM proteins with SNAREs, during which the SM protein catalyzed the formation of the SNARE complex. According to this idea and based on published work by others (Carr, Grote et al. 1999) (Hashizume, Cheng et al. 2009), this putative reaction intermediate, could only be isolated for the secretory machinery of baker's yeast while it was only transiently populated 
and less stable for the secretory proteins involved in neuronal secretion. We attempted to gain more details on the way Sec1 binds to the assembled SNARE complex, this would not only expand the knowledge we have on this particular system but also draw the parameters that are required for an interaction with a fully assembled SNARE complex, as it was proposed that this is a crucial binding mode for all the members of the SM family of proteins (Dulubova, Khvotchev et al. 2007) (Deak, Xu et al. 2009) (Sudhof and Rothman 2009)

\subsection{Biochemical characterization of the interaction between Unc18 / Unc64}

Over the last two decades, the interaction pattern of the proteins involved in neuronal secretion in the worm have been studied most intensively by genetic means. Very interesting insights were obtained when the so-called open syntaxin or LE mutant (L166A \& E167A of Unc64) was studied. At this time it was thought that the two point mutations would render Unc64 entirely open and thus expression of the LE mutant might be able to overcome the severe phenotype of the Unc18 knock-out. This, however, proved to be not correct. Remarkably, it was found instead that in the presence of the LE mutant the requirement for Unc13 in synaptic vesicles can be bypassed (Richmond, Weimer et al. 2001; Hammarlund, Palfreyman et al. 2007). As has been shown in previous studies, the SM family of proteins affects SNARE complex assembly through the interaction with the Qa-SNARE. In the C. elegans Unc18/Unc64 couple involved in neuronal secretion has been reported to work through a binding mode in which only the N-peptide of Unc64 seems to be needed for binding (McEwen and Kaplan 2008; Johnson, Ferdek et al. 2009). These insights were obtained by in vivo studies, which were accompanied by non-quantitative binding assays.

Notably, the Unc18/Unc64 binding mode appears to be somewhat in contrast to the one reported for the mammalian Munc18-1/syntaxin 1a pair. For this pair, the $\mathrm{N}$-peptide participates in binding but only contributes a smaller portion to the overall affinity of the complex. Interestingly though, the lack of the 
N-peptide seem to render Munc18-1 incapable to lock syntaxin 1a into a closed conformation that does not allow for SNARE complex formation. A recent publication also showed that interfering with the binding of the N-peptide in vivo by mutating the N-peptide binding pocket in Munc18-1 does not lead to a measurable defect in neuronal secretion (Meijer, Burkhardt et al. 2012), corroborating a difference between the two pairs. Nevertheless, this does not clarify whether there are fundamental or only subtle differences between the two pairs.

In order to investigate the contribution of the different binding regions of Unc18 and Unc64 in more detail I used Isothermal Titration Calorimetry (ITC). In order to achieve this, a series of constructs that contained mutations and truncations of Unc64 were used. The results clearly show that the N-peptide region is indeed much more important for the interaction of Unc18 and Unc64 than for the interaction of its mammalian counterparts Munc18-1 and syntaxin 1a. This indicates that the contribution of the $\mathrm{N}$-peptide region for the overall binding strength of the complex can be different for different pairs. For example; for Munc18-3 it was also found that the N-peptide of its cognate syntaxin, syntaxin 4 , contributes to binding to a large extent ( $\mathrm{Hu}$, Latham et al.). Interestingly, truncations and mutations in the SNARE motif of Unc64 severely impaired binding to Unc18. This demonstrates that the two proteins interact, like the pairs from rat and from Monosiga brevicollis, via two spatially separated binding sites. Likewise, recent data also suggest that Munc18-3 also interacts with another portion of syntaxin 4 (Christie, Whitten et al.) . In other words, the truncation experiments presented here are of particular interest as they support an interaction between Unc18 to Unc64 in a fashion that resembles the interaction between Munc18a/Syntaxin1a, this is slightly different from what was proposed in previous studies in which it was concluded that C. elegans pair only the N-peptide binding is essential for binding.

As indicated above a different approach was used to study the interaction of the two proteins in previous investigations. Clearly, the ITC approach used here allows for detection of more subtle differences compared to the binding 
assays used earlier. Yet, the overall picture did not change drastically: both approaches found that the $\mathrm{N}$-peptide region contributes significantly to the overall affinity of the Unc18/Unc64 complex and thus might indeed play a more important role as the in vivo results by (Johnson, Ferdek et al. 2009).

In fact, binding of the $\mathrm{N}$-peptide has been found to be important for hindering the bound syntaxin to form a SNARE complex. This has been found for the mammalian Munc18-1/syntaxin $1 \mathrm{a}$ complex as well as for the Munc18/syntaxin1 complex from Monosiga brevicollis, suggesting that it might constitute a conserved property of the complex. Why binding of the N-peptide is important for this transition is still unclear, however. It remains unclear whether $\mathrm{N}$-peptide binding plays a similar role for the Unc18/Unc64 complex. In fact, it has not been studied at all whether Unc18 indeed locks Unc64 in a closed conformation and by this prevents SNARE complex formation. Very likely this has been not studied as Ric4, the C. elegans SNAP-25 homolog, appears to be difficult to express in E. coli. In order to establish an assay to observe SNARE complex formation in vitro I made use of a Ric4 expression construct that was codon-optimized for E. coli expression. This allowed me to purify the protein. It turned out that the C. elegans SNAREs do not form an SDS resistant complex (Data not shown) that would make it easy to follow its assembly biochemically. As a next step it is therefore planned to label Ric4 with a fluorescent dye in order to develop an assay that allows for monitoring SNARE complex formation in real time. This assay can then be used to investigate the effect of Unc64 or Unc18 mutations. An interesting Unc64 mutation to be tested is the LE mutant, which we found to be able to bind to Unc18 albeit with reduced affinity. When the LE mutant of the mammalian syntaxin 1a was used, it was found that Munc18-1, comparable to a syntaxin 1a lacking the N-peptide region, was unable to prevent it from engaging in SNARE complex formation. It would be very interesting whether the LE mutant of Unc64 shows the same properties. 


\subsection{Mapping of interacting areas in Unc18 and Unc64 by mass spectrometry using hydroxyl radical footprinting}

The results obtained from the mass-spec analysis were focused on the Unc64 subunit. Two regions have been reported to be important in Unc64 are the N-peptide and the loop that connects the Hc-helix of the Habc domain and the SNARE helix as it has been reported that mutations in this helix render an "open" conformation that bypasses the requirement of Unc13 in C. elegans and the inhibitory effect of Munc18-1 in rat (Ma, Li et al. ; Hammarlund, Palfreyman et al. 2007). These two regions (the N-peptide and the loop) in combination with the way in which the Habc domain folds back on the SNARE motif compose the known mechanism by which SNARE assembly can be affected.

As the sequence similarities between the mammalian Munc18/Syntaxin and the nematode Unc18/Unc64 are high, it is very likely that the structure of their complexes is very similar and with this assumption, I modeled the regions shown to change upon complex formation using the crystal structure of the mammalian Munc18-1/syntaxin 1a complex (PDB:3C98). In terms of reliance in the results two regions were detected that were expected to interact: the $\mathrm{N}$ peptide (peptide 4) and the region corresponding to residues 97-115 (peptide 13), that if assigned to equivalent residues in the syntaxin found in the structure 3C98 would correspond to a region that binds to the conserved concave region formed by the Unc18 homologue Munc18 .

In the studies presented here we can add yet another interesting region that seems to show a decreased degree of modification on it side change upon complex formation and could indicate that it gets protected, this region is found between the -7 layer of the SNARE domain and the loop that connects the Hc helix of the Habc domain with the SNARE motif, and that has been shown to have regulatory properties as mutations in its sequence creates the LE open mutant. A closer inspection of this sequence region showed that it contains two highly conserved residues: Arginine 198 and a Histidine 199. The importance of these two residues is very visible when an alignment of 902 syntaxins when the degree 
of relative conservation is represented with software weblogo, the degree of relative conservation between the amino acids from the alignment is shown as relative height, the higher the more conserved. The residues in position R198 and H199 in Unc64 are as conserved as the glutamine found in the "0" layer.

In the structure of the Munc18-1 and Syntaxin 1a, the corresponding histidine (H200) physically contacts the helix that connects the Hc helix and the SNARE motif. In addition, the histidine residue contacting the helix (H200 in Syntaxin 1a, H197 in Sso1p, H213 in Syntaxin from Monosiga brevicollis) does it so in a way that resembles the rat Munc18/Syntaxin pair. This could mean that the opening of the Syntaxin could occur by removal of the helix.

This conformational change could then expose a binding region for SNAP-25, maybe allowing for first contact of the two partners SNAREs syntaxin and SNAP25 while syntaxin is still bound by Munc18. The recognition and contact site for SNAP-25 might well be the highly conserved arginine and histidine residues. In fact, binding of syntaxin and SNAP-25 has been shown to start at the N-terminal tips of their SNARE motifs (Fasshauer and Margittai 2004).

In the structure of the mammalian complex, it can be easily discerned that the helix is covering the region between residue 191-200, which includes the arginine 199 and the Histidine 200 in the complex (PDB: 3C98). One reason for why this region cannot be seen on the crystal structures of the un-complexed syntaxin $1 \mathrm{a}$ could be that it is disordered, as the four helix-bundle has been reported to be mostly in an open state (Dulubova, Yamaguchi et al. 2001; Margittai, Widengren et al. 2003). This means that syntaxin1a not only gets closed by Munc18-1 as it holds the Habc-domain over the SNARE motif, but could potentially also pull the helix so that the histidine can interact with it. If this would be the case, that the helix gets pulled so that the histidine can bind or simply get covered, then this in turn would indicate that this placement of the helix could reflect a regulatory event. So in the case of the LE mutant it can be thought that the alanine mutations remove the "pins" that keep the helix in position. 
This is more clear in the Sso1p structure where it can be seen how the helix is attached to two particular residues: Glu161that interacts with Lys 145 in Sso1p (Fig. 24B Yellow box) as the equivalent residue Glu166 interacts with Arg 142 in syntaxin1a (Fig. 24A Green box), this Glu is the glutamic acid in the L165/E166 pair that corresponds to the "open" Syntaxin. In addition to the Glu161 the yeast Sso1p contains a second residue on the helix; Asp166 that interacts with His197 (Fig. 24B Red box).

This interactions between residues on the helix and the rest of the protein could be created by the SM proteins as it binds to the Qa-SNARE, and by doing so it could make the Syntaxin inaccessible for SNARE complex formation by covering a potential recognition site and not allow any "misfiring" of the SNARE complex assembly reaction. This mechanism of inhibiting the complex formation could help explain why the LE mutant by-passes the inhibitory effect that Munc18 has on Syntaxin, and would also help explain why Sso1p is less prone to take part in the SNARE complex formation as it has two interacting residues that would need to be mutated in order for it to be "open"

A

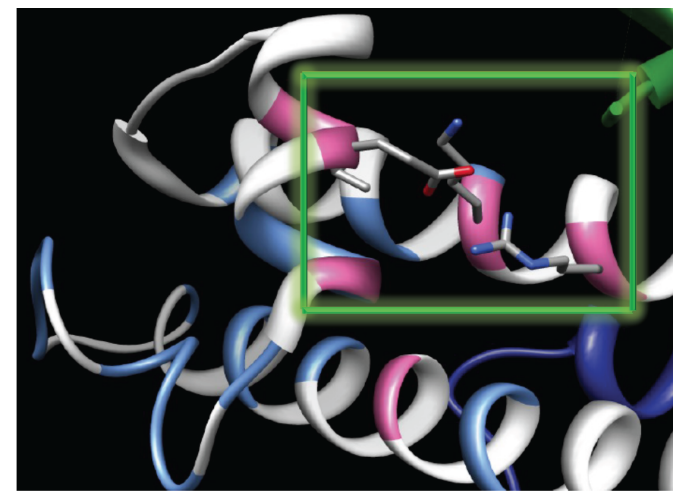

B

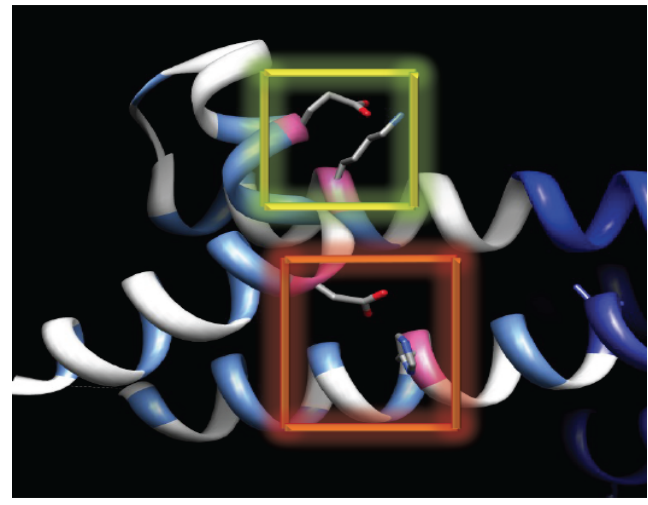

Figure 24. The loop that connects the Hc helix with the SNARE domain.

The loop that connect the Hc helix from the Habc domain makes a connection with a conserved Histidine in the vicinity of the SNARE domain just adjacent to layer -7 , this connection can be seen when Syntaxin is bound to the SM protein and thus can represent a regulatory function. When the Leucine (A green frame) in position 165 and the Glutamate in position 166 are mutated to an Alanines, the Syntaxins has been reported to bypass the regulatory roll that Munc18 provides. At the same time, the yeast Sso1p (B) has been shown to be hard to "open" and this could be explain by the fact that the loop interacts in two different places rather than one. 
That His197 plays a role in locking Sso1p in a closed conformation is supported by a report in which the conformational status of Sso1p was dependent on the pH. A pH shift from 7.0 to 9.0 led to faster assembly (Munson, Chen et al. 2000). This $\mathrm{pH}$ range is close to the pka value of the histidine side chain. In other words, a pH shift from 7.0 to 9.0 might lead to deprotonation of H199. The loss of the positive charge might remove the "pin" between H199 and the linker helix.

From our studies on the C. elegans Unc18/Unc64 interaction we can conclude that the interaction takes place in a way that resembles the Munc18a/Syntaxin1a couple, as it requires both the N-peptide and the four-helix bundle. In addition our mapping studies confirm the interaction between the $\mathrm{N}$ peptide and the four-helix bundle. In addition, drawn out attention to a region that contains a two highly conserved residues (arginine and histidine) that could be involved in creating a layer of regulation through a connection to the loop helix.

\subsection{Sec1p binding to the components of the SNARE complex}

As described above, secretion in Saccharomyces cerevisiae is mediated by a conserved molecular machinery much similar to the one used in neuronal secretion. Its core is composed of a member of the SM family of proteins, Sec1p and a recently duplicated set of secretory SNARE proteins: Sso1p/Sso2p, Sec9p/Spo20p, and Snc1p/Snc2p. Of these the Qbc-SNARE Spo20p is more derived (Kienle, Kloepper et al. 2009)) and appears to play a role during sporulation only. Several other proteins, among them a multi-protein complex named the exocyst and a small GTPase, Sec4p, play vital roles as well. Sec1p is of major interest as it is a member of the SM family that is thought to bind to the assembled SNARE complex but not directly to its cognate syntaxin Sso1p. 
Sec1p has proven to be challenging to work with, as it is very difficult to express by recombinant means. In addition, it turned out to be prone to precipitate at concentrations higher than $2 \mathrm{mg} / \mathrm{ml}$. Nevertheless, during this work I was able to develop a protocol for the production and purification of fulllength Sec1p and other variants in E. coli. Although the procedure did not yield high quantities of Sec1p, it was enough for obtaining initial data on the interaction between Sec1p and the yeast secretory SNARE proteins.

\subsubsection{Pull down studies show no interaction between Sec1p and the SNARE complex.}

To test the ability of Sec1p to bind to the individual SNARE components, to the binary Sso1p/Sec9 complex, or to the ternary SNARE complex, a series of binding experiments were carried out. Binding studies were carried out using GST-tagged Sso1p, since more unspecific binding of the SNARE proteins to the beads was observed in initial experiments using the His 6 -tag of Sec1p (data not shown).

Corroborating earlier studies Sec1p did not bind to Sso1p even after prolonged incubation times. Surprisingly, as in contrast to earlier studies, no interaction of Sec1p with the binary complex and the ternary SNARE complex was observed. So far there are only two studies that have used a comparable approach to study the interaction of Sec1p with the SNARE complex. Both studies had reached the same conclusion that Sec1p does bind to the SNARE complex.

In the report published by McNew and colleagues, Sec1p was pulled out using a GST-Sso1p construct and on first view the differences between mine and their findings appear to be striking. However, a closer inspection of their data (shown in fig. 3 (Scott, Van Komen et al. 2004)) reveal that only a very little amount of Sec1 was co-precipitated. Still, the Sec1p band in their SDS-PAGE, in contrast to mine, is clearly visible. The difference might be easily explained, 
because McNew and colleagues boiled the Glutathione-beads in SDS-sample buffer, whereas I chose to elute the precipitated proteins more specifically with glutathione.

The other studies reported by Carr and colleagues also show a substoichiometric interaction (Togneri, Cheng et al. 2006). Taken together, a closer inspection of earlier results suggests that Sec1 might only weakly, if at all, bind to the assembled SNARE complex. That Sec1p indeed does not bind tightly to Sso1p nor the assembled SNARE complex was confirmed when I used native gel electrophoresis to probe for binding. This was necessary since the yeast SNARE complex does not show a resistance to SDS as the mammalian SNARE complex.

The results obtained from the assembly studies show first and foremost that the SNARE proteins were fully capable of interacting and forming both the binary and the ternary SNARE complex. The SNARE complex formed when the SNARE motif and when the entire cytoplasmic domain of Sso1p containing the Habc domain were used.

That SNARE complex assembly was observable in the presence of the longer Sso1p construct allowed me to add Sec1p to the assembly reaction as well. First of all, no additional band, which could represent an interaction between Sec1p and the assembled SNARE complex, appeared in the presence of Sec1. First of all, this confirms the results obtained by the GST binding assay described above, suggesting that Sec1p does not interact tightly with an assembled SNARE complex. Moreover, using native gel electrophoresis no complex band between Sec1p and individual Sso1p was observed as well, corroborating that Sec1 does not tightly interact with its cognate syntaxin.

Interestingly, no influence of Sec1p on the speed of SNARE complex assembly was observed. This result is somewhat contradicting another attempt to cast light onto the effect of Sec1p on SNARE complex formation by Jantti and colleagues (Weber-Boyvat, Aro et al. 2011). They had used a similar approach but instead of full length Sec1p they only used the C-terminal "tail" of Sec1p. In 
these studies it was proposed that the tail accelerates SNARE complex formation. This cannot be excluded, but it should be noted that the C-terminal tail of Sec1p carries a highly positive net charge, which might let this region bind unspecifically bind to the yeast secretory SNAREs or the SNARE complex that has negatively charged patches on its surface.

That the tail is comprising an important region for modulating SNARE assembly appears somewhat unlikely as this domain is not present in most other SM proteins, but seems to be a new trait in the yeast lineage.

\subsubsection{Sec1p does not bind to the SNARE complex in the presence of a membrane}

As I could not detect any binding to neither the SNARE complex nor individual Sso1p by means of pull-downs nor native gel electrophoresis, I tested whether the membrane plays a role since this interaction takes place at the site of secretion in the vicinity of the plasma membrane. Indeed, the positively charged tail of Sec1p could potentially be a membrane binding device that could steer Sec1p onto the membrane and thereby promoting its interaction with the SNARE machinery. Indeed, I found that the tail of Sec1p is able to mediate its attachment to the membrane. Interestingly, the tail of Sec1 is essential for its function since a deletion of it seems to show a defect in localization (WeberBoyvat, Aro et al. 2011)

In order to assess this, 40nm liposomes were created with an Sso1p construct that contains that trans-membrane domain that is incorporated into the membrane, these liposomes were mixed with Sec1p before let to move across a Nycodenz gradient bringing with them any protein that has bound to it. The ability of Sec1p to bind to the liposomal membrane is very clear, as it is present on the top layer in every binding reaction set. 
Although Sec9p and presumably the Snc2p bind to the liposomes through Sso1p as their presence is not detected if Sso1p is not incorporated to the membrane, it is not equally clear whether Sec1p binds to Sso1p nor the SNARE complex as it is found on the upper fraction even in the absence of the membrane anchored Qa-SNARE.

Based on my studies and previously published data it is clear that probably only a very low affinity interaction or even an unspecific binding of Sec1p to Sso1p or any SNARE complex has been detected so far. My findings confirm that Sec1p does not bind to its cognate syntaxin partner Sso1p, suggesting that Sec1p is indeed somewhat different to secretory SM proteins found in animals and their unicellular ancestors. But this is, of course, not sufficient to maintain the view that Sec1 is binding to the assembled SNARE complex. In fact, so far only little evidence for this binding mode has been provided so far. Biochemically the most convincing data have been provided by Jose Rizo and colleagues, who found that Munc18-1 is able to bind to the core four-helix bundle SNARE complex. However, binding occurred with an affinity of $300 \mathrm{nM}$, which is a rather low compared to the one found for binding to syntaxin $1 \mathrm{a}(\approx 2 \mathrm{nM})$. In addition, they reported that Munc18-1 binds to the C-terminal portion of the SNARE complex, a region that is positively charged and might be very close to the membrane surface (they only used the soluble portion of the SNARE complex) (Dulubova, Khvotchev et al. 2007; Deak, Xu et al. 2009).

In order to analyze the binding mode of Sec1p qualitative approaches like ITC for example deem to be necessary. This requires that we will be able to produce and purify Sec1p in larger quantities, a task that turned out to be rather challenging. So far I have tried several strategies to overcome this hurdle, this includes expression at different temperatures and IPTG concentrations in order to slow down the expression and allow the protein to fold properly. I have also tried to express Sec1 in different E. coli strains and strategies such as the inducing chaperons in the cells by exposing the cells to both ethanol shocks and heat shocks. In addition, attempts to promote the folding by adding betaine and 
sorbitol to the media only increasing the amount purified marginally, only to precipitate short after.

As this point I'm trying three new constructs that look promising as the solubility increases drastically, especially for a construct that is composed of an area that would correspond to domain 1 in Munc18-1. Moreover, a construct lacking the C-terminal "tail" and a region between 195 - 211 has shown an increase in solubility as well.

Although these constructs have the potential of providing us with information regarding the regions involved in binding, the most interesting strategy consist in co-expressing and co-purifying Sec1p with Mso1p, as they are obligatory partners in the cell. It would be very interesting to see the difference when this component is added to the binding reaction and test its effect, since the absence Mso1p in the cell produces a phenotype that resembles the Sec1p null phenotype, that is, accumulation of secretory vesicles. But as will be argued further down, Sec1p could also be a component of a much larger protein complex that could include the already mentioned Mso1p and if not all, at least parts of the exocyst complex.

\subsection{Mso1p is an obligatory partner necessary for full Sec1p function}

Through a secretion screen and the extensive use of yeast genetics and pull-downs a suppressor of sec1-1 mutation was found (Aalto, Jantti et al. 1997). This suppressor has been reported to be a soluble protein of $17 \mathrm{kDa}$ found enriched in the microsomal membrane fraction that has the interesting property of having a pI of about 10. This protein is Mso1p and its presence is essential for secretion to take place as it has been shown by knock out studies in which the resulting phenotype is composed of accumulation of secretion vesicles that resembles the one previously observed in Sec1p deletion (Knop, Miller et al. 2005). The accumulation of secretory vesicles show that Mso1p it is involved in the terminal stage of secretion. Moreover, disruption of the Mso1p gene is lethal 
in strains that also contain mutations in the SM protein Sec1, the Guanine nucleotide exchange factor (GEF) Sec2 and the small GTPase Sec4 genes (Aalto, Jantti et al. 1997).

The role of Mso1p in assisting Sec1p in secretion has been supported by recent studies in which Mso1p has been co-localized to sites of secretion through fluorescence microscopy. In addition, it was also shown that the minimal binding surface of Mso1p to Sec1p is composed of residues 1-58 that has been mapped to interact with domain 1 of Sec1p, this region includes the $\mathrm{N}$-peptide binding site and Mso1p has been proposed to replace it since Sso1p doesn't seem to contain an N-peptide in its sequence, or at least not a canonical one (Weber, Chernov et al. 2010) (Aalto, Jantti et al. 1997) (Weber-Boyvat, Aro et al. 2011). The truncation of this region (residues 1-58) of Mso1p shows the same phenotype as the deletion of MSO1p, that is vesicle accumulation at sites of cell growth and complete inhibition of precursor vesicles in the growing pro-spore (Weber, Chernov et al. 2010).

In recent studies BiFC (Biomolecular Fluorescence Complementation) has been used to study the interaction of Sec1p to components of the secretory machinery as well as the exocyst, this method is based on the formation of a fluorescent complex through the association of two fragments of a fluorescent protein as two interacting partners bring them into proximity, and was designed to study protein interactions in living cells (Kerppola 2006).

Through BiFC studies it was shown that the interaction between Sec1p and Mso1p is unaffected in Sec18-1, Sec9-4, Sec15-1 and Sec6-4 (which are all part of the exocyst complex) and Sec4-8 strains even though Mso1p-HA fails to pull down the SNARE complex in the very same mutants at the restrictive temperature, which is the temperature at which the cells show the phenotype. In addition, re-localization of the Sec1p/Mso1p complex in Sec4-8 and Sec2-41 after 1 hour of incubation at the restrictive temperature (Knop, Miller et al. 2005 ) to the cytosol (5x), indicate a close interaction although the secretion machinery is heavily compromised in all the strains. 
When the binding region of Mso1p in Sec1p was mapped by means of random mutagenesis in combination with Yeast-2-Hybrid studies it was discovered that a group of them accumulated to a region, and when a model of Sec1p was created (Weber, Chernov et al. 2010), the mutants interested they could be grouped in a region that contains two helices in the vicinity to the hydrophobic pocket that would correspond to the N-peptide binding region, when the interaction between Mso1p and Sec1p mutants was studied using BiFC, the following effect on the signal was observed :

- Q113A (no effect on the signal)

- F115A (slightly reduced signal)*

- L125D (severely reduced signal)*

- Q113A/L125D (Abolished)*

- F115A/L125D (Abolished)*

- I111K (slightly reduced)

- Q116A (No effect on the signal)

In addition, the mutants F115A, L125D, Q113A/L125D and F115A/L125D not only show a decreased binding to Mso1p but also caused a reduced binding to Sso1p when the interaction was tested in Pull-Downs, showing a dependence of Mso1p for Sso1p binding. 


\section{Summary \& Conclusions}

At the core of the machinery that ensures that cargo vesicles fuse with their target membrane in a fast, regulated and accurate way lay the SM and SNARE proteins. SNAREs are the proteins that have been accredited with providing the raw mechanical force to pull the membranes together in order for them to fuse. The members of the SM family of proteins are thought to be responsible for regulating this event by acting in most parts on the Qa-SNAREs.

Even though SM proteins show a high degree of structural homology, three different binding modes have been described by which SM proteins bind to their cognate syntaxins (Qa-SNAREs). The three binding modes seem to correspond to an interaction with the N-terminal region of the Qa-SNARE (N-peptide) (mode1), to the close conformation of the Syntaxin (mode 2), and to the assembled SNARE complex (mode 3). Recent findings suggest that there can be a binding mode that is common to all the SM proteins. This binding mode would involve both the $\mathrm{N}$ peptide and the close conformation i. e. a combination of mode 1 and mode 2 , as has been reported is the case for the secretory SM proteins Munc18-1 and the homolog of the choanoflagellate Monosiga brevicollis.

In the case of SM protein Unc18 that is involved in neuronal secretion in $C$. elegans, it was reported to bind through the N-peptide of Unc64, while, in contrast, the yeast secretory Sec1p was found to bind to the assemble SNARE complex. On a molecular level, their interaction mode was based on semiquantitative binding assay. In order to asses this in more detail, I studied these interaction with more quantitative methods such as Isothermal Titration Calorimetry (ITC).

The results obtained from the ITC measurements for the Unc18 interaction with Unc64 indicate that the $\mathrm{N}$-peptide is indeed an essential component of the interaction, as a single point mutation in this region (L9A) reduces the affinity 
drastically. However, truncations made in the SNARE domain of Unc64 together with a point mutation introduced into the same region (I233A) revealed that the closed conformation is equally important for the interaction of Unc18 and Unc64. This strongly suggests that Unc18 binds to Unc64 in a similar manner as the mammalian Mun18-1 to syntaxin1a, suggesting that this is a more general mode of interaction. These results were confirmed by radiolytic footprinting with mass spectrometry, which is a technique that can be used to catalogue the areas that are involved in protein-protein interactions as residues become protected from modifications when in complex. As the pair Unc18/Unc64 exhibits high sequence similarity with the Munc18-1/Syntaxin1a pair, the results obtained from the Mass-spectrometry analysis were interpreted with the Munc18/Syntaxin crystal structure as an aid. The results obtained from this study confirmed that the $\mathrm{N}$-peptide is involved in the interaction, as was expected. In addition, a region that would correspond to the connecting loop between the $\mathrm{Hb}-\mathrm{Hc}$ helices and would interact with the concave region of Munc18-1. This indicates that closed conformation does play a role on the interaction as observed on the results obtained by ITC.

As it was in my interest to do a comparative study between different secretory systems, a detailed analysis was carried out on the yeast Sec1p. As Sec1 is at the center of many studies and it was found out to bind to the assembled SNARE complex rather then through the Qa-SNARE alone.

The results obtained from a series of binding studies demonstrated that Sec1p does not bind to the Qa-SNARE Sso1, nor to the binary complex composed of Sso1/Sec9 or the SNARE complex. This did not change in the presence of a membrane, although these studied revealed that the positively charge C-terminal "tail" promotes membrane binding of Sec1. Moreover, I found that the presence of Sec1p does accelerate or inhibit the SNARE complex.

Sec1 shows a very interesting binding mode that is worthy of further studies but any result obtained will require the development of stable constructs that can be produced in high enough amounts and are stable enough that methods like ITC 
can be used, or even structural studies can be tried. With this in mind I designed two different constructs that can be used for further studies. One consist of a Sec1p construct that lacks the highly positive charged "Tail" and a disordered region between residue 195-211. The other one comprises only domain 1, i. e. the region that contains the $\mathrm{N}$-peptide binding site and that has been reported to be important for binding to Mso1p, which is a factor that seems to be important for Sec1's full function. Both these constructs increased the solubility of Sec1 and can be purified by affinity chromatography. This is a big step forward since earlier attempts to obtain Sec1 through the optimization of the expression and purification conditions had failed.

As, Mso1p seems to be an obligatory component for proper secretion in yeast, I also developed a protocol for co-expression and purification of the heterodimer, as the absence of Mso1p has been reported to accumulate vesicles in the same manner as in the absence of Sec1p in yeast. This complex will be interesting to study, as it might well be the functional unit responsible for binding and regulating the secretory SNARE complex formation in baker's yeast. 


\section{Bibliography}

Aalto, M. K., J. Jantti, et al. (1997). "Mso1p: a yeast protein that functions in secretion and interacts physically and genetically with Sec1p." Proc Natl Acad Sci U S A 94(14): 7331-7336.

Antonin, W., D. Fasshauer, et al. (2002). "Crystal structure of the endosomal SNARE complex reveals common structural principles of all SNAREs." Nat Struct Biol 9(2): 107-111.

Antonin, W., C. Holroyd, et al. (2000). "A SNARE complex mediating fusion of late endosomes defines conserved properties of SNARE structure and function." Embo I 19(23): 6453-6464.

Arac, D., I. Dulubova, et al. (2005). "Three-dimensional structure of the rSly1 Nterminal domain reveals a conformational change induced by binding to syntaxin 5." J Mol Biol 346(2): 589-601.

Balch, W. E., W. G. Dunphy, et al. (1984). "Reconstitution of the transport of protein between successive compartments of the Golgi measured by the coupled incorporation of N-acetylglucosamine." Cell 39(2 Pt 1): 405-416.

Barinaga, M. (1993). "Secrets of secretion revealed." Science 260(5107): 487489.

Barlowe, C., L. Orci, et al. (1994). "COPII: a membrane coat formed by Sec proteins that drive vesicle budding from the endoplasmic reticulum." Cell 77(6): 895-907.

Baumert, M., P. R. Maycox, et al. (1989). "Synaptobrevin: an integral membrane protein of 18,000 daltons present in small synaptic vesicles of rat brain." Embo J. 8(2): 379-384.

Bennett, M. K., N. Calakos, et al. (1992). "Syntaxin: a synaptic protein implicated in docking of synaptic vesicles at presynaptic active zones." Science 257(5067): 255-259.

Bonifacino, J. S. and B. S. Glick (2004). "The mechanisms of vesicle budding and fusion." Cell 116(2): 153-166.

Bracher, A., J. Kadlec, et al. (2002). "X-ray structure of a neuronal complexinSNARE complex from squid." J Biol Chem 277(29): 26517-26523.

Bracher, A. and W. Weissenhorn (2004). "Crystal structure of the Habc domain of neuronal syntaxin from the squid Loligo pealei reveals conformational plasticity at its C-terminus." BMC Struct Biol 4(1): 6.

Brenner, S. (1974). "The genetics of Caenorhabditis elegans." Genetics 77(1): 7194.

Brummer, M. H., K. J. Kivinen, et al. (2001). "Characterization of the sec1-1 and sec1-11 mutations." Yeast 18(16): 1525-1536.

Buchan, D. W., S. M. Ward, et al. "Protein annotation and modelling servers at University College London." Nucleic Acids Res 38(Web Server issue): W563-568.

Burkhardt, P., D. A. Hattendorf, et al. (2008). "Munc18a controls SNARE assembly through its interaction with the syntaxin N-peptide." Embo I 27(7): 923933. 
Burkhardt, P., C. M. Stegmann, et al. (2012). "Primordial neurosecretory apparatus identified in the choanoflagellate Monosiga brevicollis." Proc Natl Acad Sci U S A 108(37): 15264-15269.

Burri, L., O. Varlamov, et al. (2003). "A SNARE required for retrograde transport to the endoplasmic reticulum." Proc Natl Acad Sci U S A 100(17): 98739877.

Carr, C. M., E. Grote, et al. (1999). "Sec1p binds to SNARE complexes and concentrates at sites of secretion." J Cell Biol 146(2): 333-344.

Carr, C. M. and J. Rizo "At the junction of SNARE and SM protein function." Curr Opin Cell Biol 22(4): 488-495.

Carr, C. M. and J. Rizo (2010). "At the junction of SNARE and SM protein function." Curr Opin Cell Biol.

Chen, X., J. Lu, et al. (2008). "NMR analysis of the closed conformation of syntaxin-1." J Biomol NMR 41(1): 43-54.

Christie, M. P., A. E. Whitten, et al. "Low-resolution solution structures of Munc18:Syntaxin protein complexes indicate an open binding mode driven by the Syntaxin N-peptide." Proc Natl Acad Sci U S A 109(25): 9816-9821.

Cowles, C. R., S. D. Emr, et al. (1994). "Mutations in the VPS45 gene, a SEC1 homologue, result in vacuolar protein sorting defects and accumulation of membrane vesicles." J Cell Sci 107 ( Pt 12): 3449-3459.

Crooks, G. E., G. Hon, et al. (2004). "WebLogo: a sequence logo generator." Genome Res 14(6): 1188-1190.

Dascher, C., R. Ossig, et al. (1991). "Identification and structure of four yeast genes (SLY) that are able to suppress the functional loss of YPT1, a member of the RAS superfamily." Mol Cell Biol 11(2): 872-885.

Deak, F., Y. Xu, et al. (2009). "Munc18-1 binding to the neuronal SNARE complex controls synaptic vesicle priming." I Cell Biol 184(5): 751-764.

Demircioglu, F. (2011). Comparative studies on regulation of SNARE complex formation by the SM protein Sly1p. Doctoral, Georg August University Goettingen.

Dilcher, M., B. Veith, et al. (2003). "Use1p is a yeast SNARE protein required for retrograde traffic to the ER." Embo I 22(14): 3664-3674.

Doussau, F. and G. J. Augustine (2000). "The actin cytoskeleton and neurotransmitter release: an overview." Biochimie 82(4): 353-363.

Dulubova, I., M. Khvotchev, et al. (2007). "Munc18-1 binds directly to the neuronal SNARE complex." Proc Natl Acad Sci U S A.

Dulubova, I., S. Sugita, et al. (1999). "A conformational switch in syntaxin during exocytosis: role of munc18." Embo J 18(16): 4372-4382.

Dulubova, I., T. Yamaguchi, et al. (2003). "Convergence and divergence in the mechanism of SNARE binding by Sec1/Munc18-like proteins." Proc Natl Acad Sci U S A 100(1): 32-37.

Dulubova, I., T. Yamaguchi, et al. (2002). "How Tlg2p/syntaxin 16 'snares' Vps45." Embo I 21(14): 3620-3631.

Dulubova, I., T. Yamaguchi, et al. (2001). "Vam3p structure reveals conserved and divergent properties of syntaxins." Nat Struct Biol 8(3): 258-264.

Eakle, K. A., M. Bernstein, et al. (1988). "Characterization of a component of the yeast secretion machinery: identification of the SEC18 gene product." Mol Cell Biol 8(10): 4098-4109. 
Fasshauer, D., W. Antonin, et al. (2002). "SNARE assembly and disassembly exhibit a pronounced hysteresis." Nat Struct Biol 9(2): 144-151.

Fasshauer, D., D. Bruns, et al. (1997). "A structural change occurs upon binding of syntaxin to SNAP-25." J. Biol. Chem. 7: 4582-4590.

Fasshauer, D., W. K. Eliason, et al. (1998). "Identification of a minimal core of the synaptic SNARE complex sufficient for reversible assembly and disassembly." Biochemistry 37(29): 10354-10362.

Fasshauer, D. and M. Margittai (2004). "A Transient N-terminal Interaction of SNAP-25 and Syntaxin Nucleates SNARE Assembly." J. Biol. Chem. 279(9): 7613-7621.

Fasshauer, D., H. Otto, et al. (1997). "Structural changes are associated with soluble N-ethylmaleimide- sensitive fusion protein attachment protein receptor complex formation." J. Biol. Chem. 272(44): 28036-28041.

Fasshauer, D., R. B. Sutton, et al. (1998). "Conserved structural features of the synaptic fusion complex: SNARE proteins reclassified as Q- and RSNAREs." Proc. Natl. Acad. Sci. U S A 95(26): 15781-15786.

Fernandez, I., J. Ubach, et al. (1998). "Three-dimensional structure of an evolutionarily conserved N-terminal domain of syntaxin 1A." Cell 94(6): 841-849.

Ferro-Novick, S. and R. Jahn (1994). "Vesicle fusion from yeast to man." Nature 370(6486): 191-193.

Fiebig, K. M., L. M. Rice, et al. (1999). "Folding intermediates of SNARE complex assembly." Nature Struct. Biol. 6(2): 117-123.

Foran, P., C. C. Shone, et al. (1994). "Differences in the protease activities of tetanus and botulinum $B$ toxins revealed by the cleavage of vesicleassociated membrane protein and various sized fragments." Biochemistry 33(51): 15365-15374.

Furgason, M. L., C. MacDonald, et al. (2009). "The N-terminal peptide of the syntaxin Tlg2p modulates binding of its closed conformation to Vps45p." Proc Natl Acad Sci U S A 106(34): 14303-14308.

Garcia, E. P., E. Gatti, et al. (1994). "A rat brain Sec1 homologue related to Rop and UNC18 interacts with syntaxin." Proc. Natl. Acad. Sci. U.S.A. 91(6): 2003-2007.

Gerber, S. H., J. C. Rah, et al. (2008). "Conformational switch of syntaxin-1 controls synaptic vesicle fusion." Science 321(5895): 1507-1510.

Glick, B. S. and J. E. Rothman (1987). "Possible role for fatty acyl-coenzyme A in intracellular protein transport." Nature 326(6110): 309-312.

Grabowski, R. and D. Gallwitz (1997). "High-affinity binding of the yeast cis-Golgi t-SNARE, Sed5p, to wild- type and mutant Sly1p, a modulator of transport vesicle docking." FEBS Lett 411(2-3): 169-172.

Griff, I. C., R. Schekman, et al. (1992). "The yeast SEC17 gene product is functionally equivalent to mammalian alpha-SNAP protein." J. Biol. Chem. 267(17): 12106-12115.

Grote, E., C. M. Carr, et al. (2000). "Ordering the final events in yeast exocytosis." I Cell Biol 151(2): 439-452.

Gulyas-Kovacs, A., H. de Wit, et al. (2007). "Munc18-1: sequential interactions with the fusion machinery stimulate vesicle docking and priming." I Neurosci 27(32): 8676-8686. 
Guo, W., D. Roth, et al. (1999). "The exocyst is an effector for Sec4p, targeting secretory vesicles to sites of exocytosis." EMBO J 18(4): 1071-1080.

Halachmi, N. and Z. Lev (1996). "The Sec1 family: a novel family of proteins involved in synaptic transmission and general secretion." J Neurochem 66(3): 889-897.

Hammarlund, M., M. T. Palfreyman, et al. (2007). "Open syntaxin docks synaptic vesicles." PLoS Biol 5(8): e198.

Hanson, P. I., R. Roth, et al. (1997). "Structure and conformational changes in NSF and its membrane receptor complexes visualized by quick-freeze/deepetch electron microscopy." Cell 90(3): 523-535.

Hashizume, K., Y. S. Cheng, et al. (2009). "Yeast Sec1p functions before and after vesicle docking." Mol Biol Cell 20(22): 4673-4685.

Hata, Y., C. A. Slaughter, et al. (1993). "Synaptic vesicle fusion complex contains unc-18 homologue bound to syntaxin." Nature 366(6453): 347-351.

Hata, Y. and T. C. Sudhof (1995). "A novel ubiquitous form of Munc-18 interacts with multiple syntaxins. Use of the yeast two-hybrid system to study interactions between proteins involved in membrane traffic." J Biol Chem 270(22): 13022-13028.

Hazuka, C. D., D. L. Foletti, et al. (1999). "The sec6/8 complex is located at neurite outgrowth and axonal synapse-assembly domains." J Neurosci 19(4): 1324-1334.

Hazzard, J., T. C. Sudhof, et al. (1999). "NMR analysis of the structure of synaptobrevin and of its interaction with syntaxin." J. Biomol. NMR 14(3): 203-207.

He, B. and W. Guo (2009). "The exocyst complex in polarized exocytosis." Curr Opin Cell Biol 21(4): 537-542.

Hosono, R., S. Hekimi, et al. (1992). "The unc-18 gene encodes a novel protein affecting the kinetics of acetylcholine metabolism in the nematode Caenorhabditis elegans." J Neurochem 58(4): 1517-1525.

Hsu, S. C., C. D. Hazuka, et al. (1999). "Targeting vesicles to specific sites on the plasma membrane: the role of the sec6/8 complex." Trends Cell Biol 9(4): 150-153.

Hu, S. H., C. F. Latham, et al. (2007). "Structure of the Munc18c/Syntaxin4 Npeptide complex defines universal features of the N-peptide binding mode of Sec1/Munc18 proteins." Proc Natl Acad Sci U S A 104(21): 8773-8778.

Jahn, R. and R. H. Scheller (2006). "SNAREs--engines for membrane fusion." Nat Rev Mol Cell Biol 7(9): 631-643.

Johnson, J. R., P. Ferdek, et al. (2009). "Binding of UNC-18 to the N-terminus of syntaxin is essential for neurotransmission in Caenorhabditis elegans." Biochem J 418(1): 73-80.

Katagiri, H., J. Terasaki, et al. (1995). "A novel isoform of syntaxin-binding protein homologous to yeast Sec1 expressed ubiquitously in mammalian cells." J Biol Chem 270(10): 4963-4966.

Kerppola, T. K. (2006). "Design and implementation of bimolecular fluorescence complementation (BiFC) assays for the visualization of protein interactions in living cells." Nat Protoc 1(3): 1278-1286.

Kienle, N., T. H. Kloepper, et al. (2009). "Phylogeny of the SNARE vesicle fusion machinery yields insights into the conservation of the secretory pathway in fungi." BMC Evol Biol 9(1): 19. 
Kiselar, J. G. and M. R. Chance (2010). "Future directions of structural mass spectrometry using hydroxyl radical footprinting." Journal of Mass Spectrometry 45(12): 1373-1382.

Kloepper, T. H., C. N. Kienle, et al. (2007). "An elaborate classification of SNARE proteins sheds light on the conservation of the eukaryotic endomembrane system." Mol Biol Cell 18(9): 3463-3471.

Knop, M., K. J. Miller, et al. (2005). "Molecular interactions position Mso1p, a novel PTB domain homologue, in the interface of the exocyst complex and the exocytic SNARE machinery in yeast." Mol Biol Cell 16(10): 4543-4556.

Koushika, S. P., J. E. Richmond, et al. (2001). "A post-docking role for active zone protein Rim." Nat Neurosci 4(10): 997-1005.

Latham, C. F., J. A. Lopez, et al. (2006). "Molecular dissection of the Munc18c/syntaxin4 interaction: implications for regulation of membrane trafficking." Traffic 7(10): 1408-1419.

Lerman, J. C., J. Robblee, et al. (2000). "Structural analysis of the neuronal SNARE protein syntaxin-1A." Biochemistry 39(29): 8470-8479.

Letourneur, F., E. C. Gaynor, et al. (1994). "Coatomer is essential for retrieval of dilysine-tagged proteins to the endoplasmic reticulum." Cell 79(7): 11991207.

Lewis, M. J., J. C. Rayner, et al. (1997). "A novel SNARE complex implicated in vesicle fusion with the endoplasmic reticulum." Embo I 16(11): 30173024.

Li, W., C. Ma, et al. "The crystal structure of a Munc13 C-terminal module exhibits a remarkable similarity to vesicle tethering factors." Structure 19(10): 1443-1455.

Lin, R. C. and R. H. Scheller (1997). "Structural organization of the synaptic exocytosis core complex." Neuron 19(5): 1087-1094.

Ma, C., W. Li, et al. "Munc13 mediates the transition from the closed syntaxinMunc18 complex to the SNARE complex." Nat Struct Mol Biol 18(5): 542549.

MacDonald, C., M. Munson, et al. (2010). "Autoinhibition of SNARE complex assembly by a conformational switch represents a conserved feature of syntaxins." Biochem Soc Trans 38(Pt 1): 209-212.

Malsam, J., F. Seiler, et al. (2009). "The carboxy-terminal domain of complexin I stimulates liposome fusion." Proc Natl Acad Sci U S A 106(6): 2001-2006.

Margittai, M. and R. Langen (2004). "Template-assisted filament growth by parallel stacking of tau." Proc Natl Acad Sci U S A 101(28): 10278-10283.

Margittai, M., J. Widengren, et al. (2003). "Single-molecule fluorescence resonance energy transfer reveals a dynamic equilibrium between closed and open conformations of syntaxin 1." Proc Natl Acad Sci U S A.

McEwen, J. M. and J. M. Kaplan (2008). "UNC-18 promotes both the anterograde trafficking and synaptic function of syntaxin." Molecular Biology of the Cell 19(9): 3836-3846.

McNew, J. A., F. Parlati, et al. (2000). "Compartmental specificity of cellular membrane fusion encoded in SNARE proteins [In Process Citation]." Nature 407(6801): 153-159.

Meijer, M., P. Burkhardt, et al. (2012). "Munc18-1 mutations that strongly impair SNARE-complex binding support normal synaptic transmission." EMBO I 31(9): 2156-2168. 
Melia, T. J., T. Weber, et al. (2002). "Regulation of membrane fusion by the membrane-proximal coil of the t-SNARE during zippering of SNAREpins." J Cell Biol 158(5): 929-940.

Misura, K. M., R. H. Scheller, et al. (2000). "Three-dimensional structure of the neuronal-Sec1-syntaxin 1a complex." Nature 404(6776): 355-362.

Munson, M., X. Chen, et al. (2000). "Interactions within the yeast t-SNARE sso1p that control SNARE complex assembly." Nature Struct. Biol. 7(10): 894902.

Munson, M. and F. M. Hughson (2002). "Conformational regulation of SNARE assembly and disassembly in vivo." J Biol Chem 277(11): 9375-9381.

Nicholson, K. L., M. Munson, et al. (1998). "Regulation of SNARE complex assembly by an N-terminal domain of the t- SNARE Sso1p." Nature Struct. Biol. 5(9): 793-802.

Nicholson, K. L., M. Munson, et al. (1998). "Regulation of SNARE complex assembly by an N-terminal domain of the t-SNARE Sso1p." Nat Struct Biol 5(9): 793-802.

Novick, P., C. Field, et al. (1980). "Identification of 23 complementation groups required for post- translational events in the yeast secretory pathway." Cell 21(1): 205-215.

Oganesyan, N., I. Ankoudinova, et al. (2007). "Effect of osmotic stress and heat shock in recombinant protein overexpression and crystallization." Protein Expr Purif 52(2): 280-285.

Ogawa, H., S. Harada, et al. (1998). "Functional properties of the unc-64 gene encoding a Caenorhabditis elegans syntaxin." J Biol Chem 273(4): 21922198.

Ossig, R., C. Dascher, et al. (1991). "The yeast SLY gene products, suppressors of defects in the essential GTP-binding Ypt1 protein, may act in endoplasmic reticulum-to-Golgi transport [see comments]." Mol Cell Biol 11(6): 29802993.

Oyler, G. A., G. A. Higgins, et al. (1989). "The identification of a novel synaptosomal-associated protein, SNAP-25, differentially expressed by neuronal subpopulations." J Cell Biol 109(6 Pt 1): 3039-3052.

Palade, G. (1975). "Intracellular aspects of the process of protein synthesis." Science 189(4200): 347-358.

Pearse, B. M. (1975). "Coated vesicles from pig brain: purification and biochemical characterization." J Mol Biol 97(1): 93-98.

Peng, R. and D. Gallwitz (2002). "Sly1 protein bound to Golgi syntaxin Sed5p allows assembly and contributes to specificity of SNARE fusion complexes." J Cell Biol 157(4): 645-655.

Peng, R. and D. Gallwitz (2004). "Multiple SNARE interactions of an SM protein: Sed5p/Sly1p binding is dispensable for transport." Embo I 23(20): 39393949.

Pevsner, J., S. C. Hsu, et al. (1994). "Specificity and regulation of a synaptic vesicle docking complex." Neuron 13(2): 353-361.

Pevsner, J., S. C. Hsu, et al. (1994). "n-Sec1: a neural-specific syntaxin-binding protein." Proceedings of the National Academy of Sciences of the United States of America 91(4): 1445-1449.

Pobbati, A., A. Stein, et al. (2006). "N- to C-terminal SNARE complex assembly promotes rapid membrane fusion." Science 313(5787): 673-676. 
Rathore, S. S., E. G. Bend, et al. "Syntaxin N-terminal peptide motif is an initiation factor for the assembly of the SNARE-Sec1/Munc18 membrane fusion complex." Proc Natl Acad Sci U S A 107(52): 22399-22406.

Richmond, J. E., R. M. Weimer, et al. (2001). "An open form of syntaxin bypasses the requirement for UNC-13 in vesicle priming." Nature 412(6844): 338341.

Rizo, J. and C. Rosenmund (2008). "Synaptic vesicle fusion." Nat Struct Mol Biol 15(7): 665-674.

Rizo, J. and T. C. Sudhof (2002). "Snares and Munc18 in synaptic vesicle fusion." Nat Rev Neurosci 3(8): 641-653.

Robinson, J. S., D. J. Klionsky, et al. (1988). "Protein sorting in Saccharomyces cerevisiae: isolation of mutants defective in the delivery and processing of multiple vacuolar hydrolases." Mol Cell Biol 8(11): 4936-4948.

Roth, T. F. and K. R. Porter (1964). "Yolk Protein Uptake in the Oocyte of the Mosquito Aedes Aegypti. L." J Cell Biol 20: 313-332.

Rothman, J. E. (1994). "Mechanisms of intracellular protein transport." Nature 372(6501): 55-63.

Salminen, A. and P. J. Novick (1989). "The Sec15 protein responds to the function of the GTP binding protein, Sec4, to control vesicular traffic in yeast." I Cell Biol 109(3): 1023-1036.

Salzberg, A., N. Cohen, et al. (1993). "The Drosophila Ras2 and Rop gene pair: a dual homology with a yeast Ras-like gene and a suppressor of its loss-offunction phenotype." Development 117(4): 1309-1319.

Sandee, D., S. Tungpradabkul, et al. (2005). "Combination of Dsb coexpression and an addition of sorbitol markedly enhanced soluble expression of single-chain Fv in Escherichia coli." Biotechnol Bioeng 91(4): 418-424.

Scott, B. L., J. S. Van Komen, et al. (2004). "Sec1p directly stimulates SNAREmediated membrane fusion in vitro." J Cell Biol 167(1): 75-85.

Shen, J., D. C. Tareste, et al. (2007). "Selective Activation of Cognate SNAREpins by Sec1/Munc18 Proteins." Cell 128(1): 183-195.

Shin, D. M., X. S. Zhao, et al. (2000). "The mammalian Sec6/8 complex interacts with $\mathrm{Ca}(2+)$ signaling complexes and regulates their activity." I Cell Biol 150(5): 1101-1112.

Söllner, T., M. K. Bennett, et al. (1993). "A protein assembly-disassembly pathway in vitro that may correspond to sequential steps of synaptic vesicle docking, activation, and fusion." Cell 75(3): 409-418.

Stein, A., G. Weber, et al. (2009). "Helical extension of the neuronal SNARE complex into the membrane." Nature 460(7254): 525-528.

Strop, P., S. E. Kaiser, et al. (2008). "The structure of the yeast plasma membrane SNARE complex reveals destabilizing water-filled cavities." I Biol Chem 283(2): 1113-1119.

Sudhof, T. C. and J. E. Rothman (2009). "Membrane fusion: grappling with SNARE and SM proteins." Science 323(5913): 474-477.

Sutton, R. B., D. Fasshauer, et al. (1998). "Crystal structure of a SNARE complex involved in synaptic exocytosis at 2.4 A resolution." Nature 395(6700): 347-353.

Sweet, D. J. and H. R. Pelham (1993). "The TIP1 gene of Saccharomyces cerevisiae encodes an $80 \mathrm{kDa}$ cytoplasmic protein that interacts with the cytoplasmic domain of Sec20p." Embo I 12(7): 2831-2840. 
Takamoto, K. and M. R. Chance (2006). "Radiolytic protein footprinting with mass spectrometry to probe the structure of macromolecular complexes." Annu Rev Biophys Biomol Struct 35: 251-276.

Tellam, J. T., S. L. Macaulay, et al. (1997). "Characterization of Munc-18c and syntaxin-4 in 3T3-L1 adipocytes. Putative role in insulin-dependent movement of GLUT-4." J Biol Chem 272(10): 6179-6186.

Togneri, J., Y. S. Cheng, et al. (2006). "Specific SNARE complex binding mode of the Sec1/Munc-18 protein, Sec1p." Proc Natl Acad Sci U S A 103(47): 17730-17735.

Toonen, R. F. and M. Verhage (2003). "Vesicle trafficking: pleasure and pain from SM genes." Trends Cell Biol 13(4): 177-186.

Toonen, R. F. and M. Verhage (2007). "Munc18-1 in secretion: lonely Munc joins SNARE team and takes control." Trends Neurosci 30(11): 564-572.

Trimble, W. S., D. M. Cowan, et al. (1988). "VAMP-1: a synaptic vesicle-associated integral membrane protein." Proc. Natl. Acad. Sci. USA 85: 4538-4542.

Verhage, M., A. S. Maia, et al. (2000). "Synaptic assembly of the brain in the absence of neurotransmitter secretion." Science 287(5454): 864-869.

Verhage, M. and R. F. Toonen (2007). "Regulated exocytosis: merging ideas on fusing membranes." Curr Opin Cell Biol 19(4): 402-408.

Voets, T., R. F. Toonen, et al. (2001). "Munc18-1 promotes large dense-core vesicle docking." Neuron 31(4): 581-591.

Wada, Y., K. Kitamoto, et al. (1990). "The SLP1 gene of Saccharomyces cerevisiae is essential for vacuolar morphogenesis and function." Mol Cell Biol 10(5): 2214-2223.

Wang, L. W. and M. R. Chance (2011). "Structural Mass Spectrometry of Proteins Using Hydroxyl Radical Based Protein Footprinting." Analytical Chemistry 83(19): 7234-7241.

Weber, M., K. Chernov, et al. (2010). "Mso1p regulates membrane fusion through interactions with the putative N-peptide-binding area in Sec1p domain 1." Mol Biol Cell 21(8): 1362-1374.

Weber, M., K. Chernov, et al. (2010). "Mso1p Regulates Membrane Fusion through Interactions with the Putative N-Peptide Binding Area in Sec1p Domain 1 " Mol Bio Cell: in press.

Weber, T., B. V. Zemelman, et al. (1998). "SNAREpins: minimal machinery for membrane fusion." Cell 92(6): 759-772.

Weber-Boyvat, M., N. Aro, et al. (2011). "Sec1p and Mso1p C-terminal tails cooperate with the SNAREs and Sec4p in polarized exocytosis." Mol Biol Cell 22(2): 230-244.

Wickner, W. and A. Haas (2000). "Yeast homotypic vacuole fusion: a window on organelle trafficking mechanisms." Annu Rev Biochem 69: 247-275.

Wiederhold, K. and D. Fasshauer (2009). "Is assembly of the SNARE complex enough to fuel membrane fusion?" J. Biol. Chem. 284: 13143-13152.

Wilson, D. W., C. A. Wilcox, et al. (1989). "A fusion protein required for vesiclemediated transport in both mammalian cells and yeast." Nature 339(6223): 355-359.

$\mathrm{Xu}, \mathrm{Y} ., \mathrm{A}$. B. Seven, et al. "Membrane bridging and hemifusion by denaturated Munc18." PLoS One 6(7): e22012.

Yamaguchi, T., I. Dulubova, et al. (2002). "Sly1 binds to Golgi and ER syntaxins via a conserved N-terminal peptide motif." Dev Cell 2(3): 295-305. 
Yang, B., M. Steegmaier, et al. (2000). "nSec1 binds a closed conformation of syntaxin1A." J Cell Biol 148(2): 247-252.

Yeaman, C., K. K. Grindstaff, et al. (2001). "Sec6/8 complexes on trans-Golgi network and plasma membrane regulate late stages of exocytosis in mammalian cells." J Cell Biol 155(4): 593-604.

Yoshida, S., Y. Ohya, et al. (1995). "STT10, a novel class-D VPS yeast gene required for osmotic integrity related to the PKC1/STT1 protein kinase pathway." Gene 160(1): 117-122.

$\mathrm{Yu}, \mathrm{I}$. M. and F. M. Hughson "Tethering factors as organizers of intracellular vesicular traffic." Annu Rev Cell Dev Biol 26: 137-156.

Zhang, F., Y. Chen, et al. (2004). "SNARE assembly and membrane fusion, a kinetic analysis." J Biol Chem 279(37): 38668-38672.

Zilly, F. E., J. B. Sorensen, et al. (2006). "Munc18-bound syntaxin readily forms SNARE complexes with synaptobrevin in native plasma membranes." PLoS Biol 4(10): e330.

Zwilling, D., A. Cypionka, et al. (2007). "Early endosomal SNAREs form a structurally conserved SNARE complex and fuse liposomes with multiple topologies." Embo I 26(1): 9-18. 


\section{Appendix}

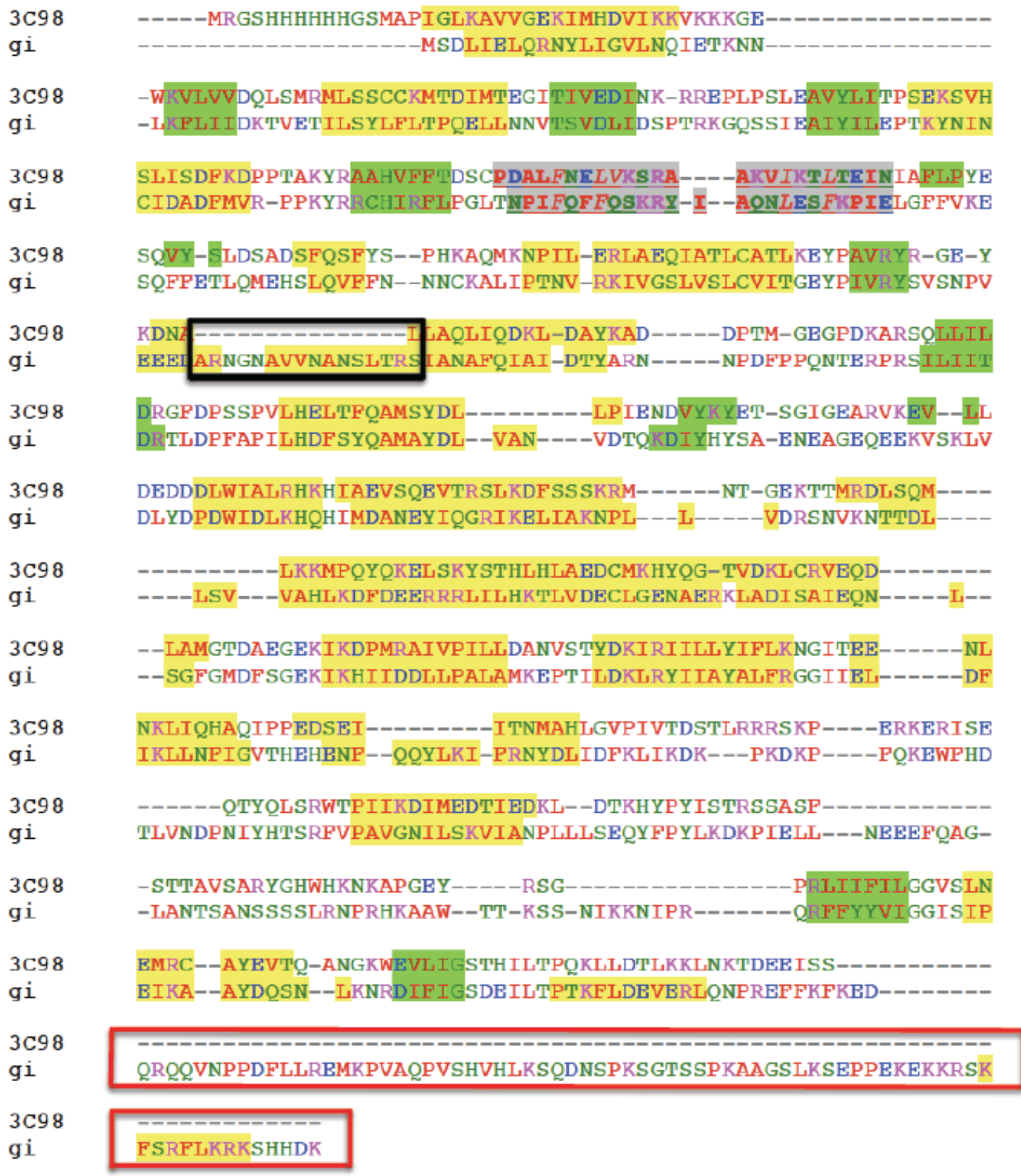

\section{Appendix 1. secondary structure prediction between Munc18-1 and Sec1p}

By using the software PSIPRED a secondary structure study was done on both Munc18-1 and Sec1p and compared. Before the comparison was made the prediction obtained for Munc18 was compared to the solved crystal structure for assurance. The comparison shows that even though the sequence conservation is poor, the secondary structure seems to be conserved, according to this estimations it is easier to navigate along the Sec1p sequence. 


\section{Hydroxyl radical footprinting experiments}

The experiments to analyze the binding surfaces by means of Hydroxyl radical footprinting in combination with Mass-spec were performed in full by Romina Hofele at the Max-Planck Institute for biophysical chemistry, Bioanalytical Mass Spectrometry group.

\section{Alignment of QaIV SNAREs}

The alignment of the Qa.IV SNARE were produce with MUSCLE and kindly provided by Dr. Kloepper and Dr. Kienle 
Education Oct 2008 - Present_Doctoral Program

Max-Planck Institute for Biophysical Chemistry, Göttingen

Biomolecules: Structure, Function and Dynamics Program

Graduate School for Neurosciences and Molecular Biosciences (GGNB)

1999 - 2004 Bachelor's/Master's Program

Natural Science program, Chemistry

Uppsala University, Sweden

MSc Degree project performed at The Sanford-Burnham Institute, La Jolla CA, USA

\section{Scholarships/Awards}

- $\quad$ The SURF Program fellowship award. Mayo Clinic, USA

\section{Publications}

Derunes, C., Burgess, R., Iraheta, E., Becherer, K., Gessner, C.R., Li, S., Hewitt, K., Vuori, K., Pasquale, E.B., Woods, Jr., V.L. and Ely, K.R. Molecular determinants for interaction of SHEP1 with Cas localize to a highly solvent-protected region in the complex. FEBS Lett. 2006 Jan 9;580(1):175-8. Epub 2005 Dec 12.

\section{Research experience}

Oct 2008 - present Max-Planck Insitute for Biophysical Chemistry, Department of Neurobiology Graduate Student

Sept 2007- Sept 2008. University of Oxford, Department of Physiology Anatomy and Genetics Research Technician 
Sept 2006- Oct 2007

June 2002- September 2002.

June 2001- September 2001.
University of Oxford, Laboratory of Molecular

Biophysics

Laboratory manager

The Burnham Institute, Department of structural Biology

Masters Degree project in structural biology

Mayo Clinic, Department of Experimental

Pathology

Special Project Associate

Mayo Clinic, Department of Biochemistry

Summer Research Student 